NBSIR $82-2454$

Polarized Tagged Photons An Analysis of the Differential Cross Section for Polarized Bremsstrahlung in the Range of Interest for a Tagged Photon System

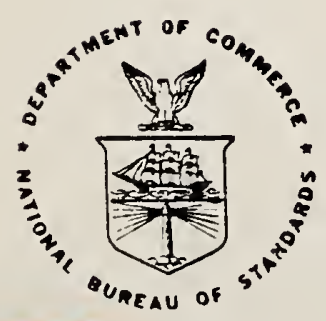




\section{POLARIZED TAGGED PHOTONS}

AN ANALYSIS OF THE DIFFERENTIAL

CROSS SECTION FOR POLARIZED

BREMSSTRAHLUNG IN THE RANGE OF INTEREST FOR A TAGGED PHOTON

\section{SYSTEM}

Leonard C. Maximon

U.S. DEPARTMENT OF COMMERCE

National Bureau of Standards

Center for Radiation Research

Nuclear Radiation Division

Washington, DC 20234

Eric Ganz

Physics Department

Stanford University

Stanford, California 94305

Thierry Aniel and Arlette de Miniac

Centre d'Etudes Nucleaires de Saclay

Department de Physique Nucleaire

Service de la Metrologie et

de la Physique Neutroniques Fondamentales

91191 Gif-sur-Yvette Cedex, France

January 1982

U.S. DEPARTMENT OF COMMERCE, Malcolm Baldrige, Secretary NATIONAL BUREAU OF STANDARDS, Ernest Ambler, Director 


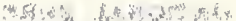

Yout A

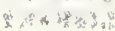

a 78 


\section{POLARIZED TAGGED PHOTONS}

An analysis of the differential cross section for polarized bremsstrahlung in the range of interest for a tagged photon system

Leonard C. Maximon

Nuclear Radiation Division

Center for Radiation Research

National Bureau of Standards

Washington, D.C. 20234

Eric Ganz

Physics Department

Stanford University

Stanford, California 94305

Thierry Aniel and Arlette de Miniac

Centre d'Etudes Nucléaires de Saclay

Départment de Physique Nucléaire

Service de la Métrologie et

de la Physique Neutroniques Fondamentales

91191 Gif-sur-Yvette Cedex, France

\section{ABSTRACT}

We consider in detail the differential cross section for polarized bremsstrahlung for angles and energies in the range of interest for a tagging system and deriye a high energy, small angle approximation for this cross section. For photons polarized perpendicular and parallel to the production plane these are given by eqs (I.2) and (I.3). We use these approximations to determine the maxima and minima of the cross sections for these two polarization states, do, and do $\sigma_{1}$ and to evaluate these cross sections at the extrema. It is showh that both $d \sigma$ and $d \sigma_{1}$ have a very sharp dip in the region of small momentum transfers. Howeyer, their behavior in the region of the dip, as a function of the azimuthal angle $\phi$, is quite different over most of the photon spectrum (condition (VI.33l). The cross section do, behaves similarly to the cross section for unpolarized photons in that as $\phi$ increases, the sharp dip yanishes, the minimum fuses with the second maximum; and the cross section then has only a single maximum. In contrast, the sharp dip in the cross section $d \sigma_{\|}$remains as $\phi$ increases, provided condition (VI.33) is satisfied. This results in rather large polarizations in the region of the dip as shown in figs. $3(a)-3(h)$. Coulomb corrections to the Born approximation are considered, and do not fill in these dips.

Key Words: Bethe-Heitler cross section; bremsstrahlung monochromator; photonuclear research; polarized bremsstrahlung differential cross section; polarized photon beams; tagged photon method. 


\section{ACKNOWLEDGEMENTS}

We wish to express our gratitude to Gloria Wiersma, to whom we are indebted for the skillful and expeditious typing of this report. We thank Roland Bergère and Larry Cardman for their heipful discussions, encouragement, aid and counsel. One of us (L. C. M.) wishes to thank Roland Bergère for the gracious hospitality accorded him during his stay at the Centre d'Etudes Nucléaires de Saclay, where this work was done. 
TABLE OF CONTENTS

ABSTRACT ........................

ACKNOWLEDGEMENTS ....................... i

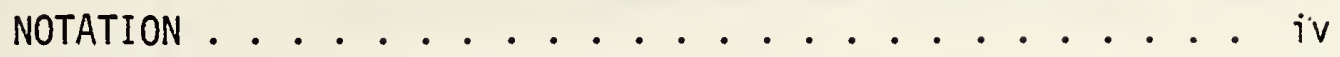

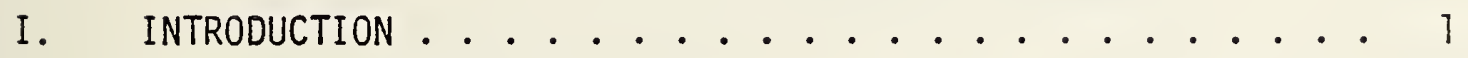

II. POLARIZATION ................ 6

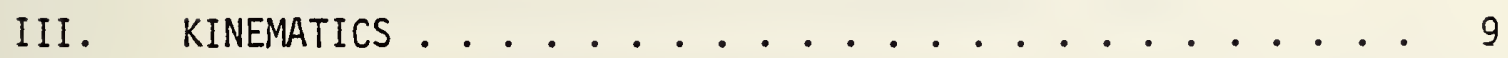

IV. BORN APPROXIMATION DIFFERENTIAL CROSS SECTION FOR POLARIZED PHOTONS EMITTED BY UNPOLARIZED ELECTRONS . . . . 12

V. HIGH ENERGY SMALL ANGLE APPROXIMATION TO THE DIFFERENTIAL CROSS SECTION FOR POLARIZED PHOTONS . . . . 19

VI. DETERMINATION OF THE MAXIMA AND MINIMUM OF THE POLARIZED BREMSSTRAHLUNG CROSS SECTIONS $d \sigma_{\perp}$ AND $d \sigma_{\|} \cdot{ } \cdot 47$

VII. COULOMB CORRECTIONS TO THE DIFFERENTIAL CROSS SECTION FOR POLARIZED BREMSSTRAHLUNG . . . . . . . . . . 66

REFERENCES ...................... 68

FIGURE CAPTIONS ................... 70

FIGURES ........................ 75 


\section{NOTATION}

\section{Physical Constants}

e charge of the electron
$m \quad$ mass of the electron
$c \quad$ velocity of light
$\mathrm{mc}^{2} \quad$ rest energy of the electron
$\hbar \quad$ atomic number of the nucleus

\section{Kinematics and Cross Section}

Note: Unless otherwise specified, all energies are in units of $\mathrm{mc}^{2}$ and a 11 momenta are in units of $\mathrm{mc}$.

k momentum of the photon

$k \quad$ photon energy

p momentum of the electron

$\underline{p}_{1}$ for the initial electron

$\underline{\mathrm{p}}_{2}$ for the final electron

$p_{\perp} \quad$ component of $p$ perpendicular to $\underline{k}$

$p_{11}$ for the initial electron

$p_{21}$ for the final electron

$$
\begin{aligned}
& p_{1}=|p| \quad \text { magnitude of } p \\
& p_{1} \text { for the initial electron } \\
& p_{2} \text { for the final electron }
\end{aligned}
$$


$\varepsilon$

electron energy

$\varepsilon_{1}$ for the initial electron ( $E_{7}$, in $\mathrm{MeV}$, in the figures)

$\varepsilon_{2}$ for the final electron

polar angle in system with $z$-axis in the direction of $\underline{k}$ $\theta_{1}$ between the photon and the initial electron direction $\theta_{2}$ between the photon and the final electron direction

$\phi$

azimuthal angle between the plane defined by the directions of the photon and the initial electron and the plane defined by the directions of the photon and the final electron

q

momentum transferred to the nucleus

$q_{1}$ component perpendicular to the photon direction

$q_{z}$ component in the photon direction

$q \quad$ magnitude of $q$

$F(q)$ atomic form factor for screening ( $=0$ in this report)

e polarization vector of the photon

$\psi \quad$ angle between $\underline{\mathrm{e}}$ and the production plane defined by the directions $\underline{k}$ and $\underline{p}_{1}$

$d \Omega_{k} \quad$ solid angle in which the photon is emitted

$d \Omega p_{2}$

solid angle in which the final electron is emitted

dk

energy bin of the photon in $\mathrm{mc}^{2}$

differential cross section for bremsstrahlung in units of $b /(s r)^{2} /\left(m c^{2}\right)$ 
Special Notations of this Report

$d \sigma_{\psi} \equiv d^{3} \sigma \equiv \frac{d^{3} \sigma}{d \Omega_{p_{2}} d \Omega_{k} d k} \quad \begin{aligned} & \text { differential cross section for bremsstrahlung } \\ & \text { for an angle of polarization } \psi\end{aligned}$

$d \sigma_{\perp} \equiv d^{3} \sigma_{\perp} \equiv \frac{d^{3} \sigma\left(\psi=\frac{\pi}{2}\right)}{d \Omega_{p} d \Omega_{2} d k} \quad \begin{aligned} & \text { value of } d \sigma_{\psi} \\ & \text { polarization) }\end{aligned}$ for $\psi=\frac{\pi}{2} \quad$ (perpendicular

$d \sigma_{\|} \equiv d^{3} \sigma_{\|} \equiv \frac{d^{3} \sigma(\psi=0)}{d \Omega_{p_{2}} d \Omega_{k} d k}$ yalue of $d \sigma_{\psi}$ for $\psi=0$ (parallel polarization)

\{\} quantity defined by $d^{3} \sigma=z^{2} \frac{e^{2}}{\hbar c}\left(\frac{e^{2}}{m c^{2}}\right)^{2} \frac{[1-F(q)]^{2}}{q^{4}} \frac{1}{k} \frac{p_{2}}{p_{1}}\left(\frac{1}{2 \pi}\right)^{2}\{\}$

A, B, C quantities defined by \{\}$=A \cos \psi+2 B \cos \psi \sin \psi+C \sin ^{2} \psi$ (exact expressions are given in eq (IV.11) page 17).

\{\}$_{1}=C$ yalue of \{\} for $\psi=\frac{\pi}{2}$
\{\}$_{\|}=A$ value of \{\} for $\psi=0$

$P_{\psi} \quad$ degree of polarization, $\quad P_{\psi}=\frac{d \sigma_{\psi}-d \sigma_{\psi+\frac{1}{2} \pi}}{d \sigma_{\psi}+d \sigma_{\psi+\frac{1}{2} \pi}}$

$P \quad$ value of $P_{\psi}$ for $\psi=\frac{\pi}{2}, \quad P=\frac{d \sigma_{\perp}-d \sigma_{\|}}{d \sigma_{\perp}+d \sigma_{\|}}=\frac{\{\}_{\perp}-\{\}_{\|}}{\{\}_{\perp}+\{\}_{\|}}=\frac{C-A}{C+A}$

$\psi_{0} \quad$ angle $\psi$ for which $P_{\psi}$ reaches $i$ ts maximum for fixed values of $\varepsilon_{1}, k, \theta_{1}, \theta_{2}, \phi$ 


$$
\begin{aligned}
& \underline{u} \equiv p_{11} \\
& \underline{v} \equiv p_{21} \\
& u \equiv|\underline{u}|=p_{1} \sin \theta_{1} \quad \text { magnitude of } \underline{u} \\
& \underline{v} \equiv|\underline{y}|=p_{2} \sin \theta_{2} \quad \text { magnitude of } \underline{v} \\
& z=\frac{y-u}{u} \\
& d_{1}=\varepsilon_{1}-p_{1} \cos \theta_{1} \\
& d_{2}=\varepsilon_{2}-p_{2} \cos \theta_{2} \\
& \xi=\frac{1}{2 \varepsilon \eta^{d}} \\
& \eta=\frac{1}{2 \varepsilon_{2} d_{2}} \\
& \xi_{0}=\frac{1}{\left(1+u^{2}\right)} \\
& n_{0}=\frac{1}{\left(1+v^{2}\right)} \\
& \xi_{1}=\xi_{0}-\xi \\
& n_{1}=n_{0}-n \\
& \delta=\frac{k}{2 \varepsilon_{1} \varepsilon_{2}}
\end{aligned}
$$




$\left.\begin{array}{c}f(v) \\ g(v) \\ \sigma \\ \rho\end{array}\right\}$

quantities defined by \{\}$=f(v)(y-u+\sigma)^{2}+g(v) \sin ^{2} \frac{\phi}{2}+o$

$f_{1}(v)$ value of $f(y)$ for $\psi=\frac{\pi}{2}$, given in eq (v.38) p. 45

$f_{\|}(v)$ yalue of $f(y)$ for $\psi=0$, given in eq $(v .41)$ p. 45

$g_{1}$ (y) yalue of $g(y)$ for $\psi=\frac{\pi}{2}$, given in eq (V.39) p. 45; (approximation for $q \leqq O(u \theta)$ and $\phi \ll 1$ given in eq (VI.4) p. 48)

$g_{\|}(y)$ yalue of $g(v)$ for $\psi=0$, given in eq $(v .42)$ p. 45 ; (approximation for $q \leqq 0(u \theta)$ and $\phi \ll 1$ given in eq (VI.5) p. 48)

$\sigma_{\perp} \quad$ is zero

$\sigma_{\text {II }}$ value of $\sigma$ for $\psi=0$, given in eq (V.43) p. 46; (approximation for $q \leqq 0(u \theta)$ and $\phi \ll 1$ given in eq (VI.6) p. 49)

$\rho_{1} \quad$ is zero

III value of $\rho$ for $\psi=0$, given in eq $(v .44)$ p. 46 $f^{\prime}(u)=\left.\frac{\partial f(v)}{\partial y}\right|_{y=u} \begin{aligned} & \text { yalue of the derivative of } f(v) \text { with respect to } \\ & \text { the variable } v \text { at the point } v=u\end{aligned}$

$$
\begin{aligned}
& f_{1}^{\prime}(u) \text { value of } f^{\prime}(u) \text { for } \psi=\frac{\pi}{2} \\
& f_{\| l}^{\prime}(u) \text { value of } f^{\prime}(u) \text { for } \psi=0
\end{aligned}
$$

$g^{\prime}(u)=\left.\frac{\partial g(y)}{\partial y}\right|_{y=u} \begin{aligned} & \text { yalue of the deriyative function of } g(v) \text { with } \\ & \text { respect to the variable } v \text { at the point } y=u\end{aligned}$

$$
\begin{aligned}
& g_{1}^{\prime}(u) \text { value of } g^{\prime}(u) \text { for } \psi=\frac{\pi}{2} \\
& g_{\|}^{\prime}\left(u L \text { value of } g^{\prime}(u) \text { for } \psi=0\right.
\end{aligned}
$$


$k_{0} \quad$ critical yalue of $k, \quad k_{0}{ }^{2}=4 \varepsilon_{1} \varepsilon_{2}\left(\frac{u^{2}-1}{u^{2}+1}\right)^{2}$

$\phi_{c} \quad$ critical value of $\phi$ for which the minimum and the second maximum of the unpolarized cross section fuse in an inflection point

$\phi_{0} \quad$ when $\phi$ approaches the value $\phi_{0}$, the approximation for the unpolarized cross section becomes invalid

$\phi_{C l}$ same as $\phi_{C}$ but for perpendicularly polarized cross section

$\phi_{\text {al }}$ same as $\phi_{0}$ but for perpendicularly polarized cross section, expression given in eq (VI.16I p. 53

$\phi_{C l l} \quad$ same as $\phi_{C}$ but for parallel polarized cross section

$\phi_{\text {oll }} \quad$ same as $\phi_{0}$ but for parallel polarized cross section, used for $k>k_{0}$; expression is given in eq (VI.26) p. 58

$\bar{\phi}_{\text {oll }} \quad$ same as $\phi_{0}$ but for parallel polarized cross section, used for $k<k_{0}$

$z_{\min } \quad$ approximation for the position of the minimum of the unpolarized cross section in the variable $z$ in the region $z=0\left(\theta^{2}\right)$

$z_{\text {minl }}$ same as $z_{\min }$ but for perpendicularly polarized cross section, expression given in eq (VI.18) p. 53

$z_{\text {minll }}$ same as $z_{\min }$ but for parallel polarized cross section; expression available for $k>k_{0}$, given in eq (VI.28) p. 59. For $k<k_{0}$ replace $\phi_{\text {oll }}{ }^{2}$ by ${ }^{\prime}-\bar{\phi}_{\text {oll }}{ }^{2}$ 
$z_{\max }$

approximation for the positions of the maxima of the unpolarized cross section in the region $z=0\left(\theta^{2}\right)$

$z_{\operatorname{max\perp }} \quad$ same as $z_{\max }$ but for perpendicularly polarized cross section, expression given in eq (VI.20) p. 55

$z_{\max } \quad$ same as $z_{\max }$ but for parallel polarized cross section, expression given in eq (VI.30) p. 61

$\lambda_{1}^{(2)} \quad$ quantity appearing in the expression of $z_{\max \perp}$, expression given in eq (VI.22) p. 56

$\lambda_{\|}^{(2)} \quad$ quantity appearing in the expression of $z_{\max \|}$, expression given in eq (VI.32) p. 61

$z_{\operatorname{max\perp }}^{(0)} \quad$ zeroth order approximation for $z_{\operatorname{maxl}}$, expression given in eq (VI.19) p. 55

$z_{\max }^{(0)} \quad$ zeroth order approximation for $z_{\max \|}$, expression given in eq (VI.29) p. 60 


\section{INTRODUCTION}

In a recent report $[1]^{1}$ we presented a detailed analysis of the differential cross section for tagged photons in the range of energies and angles of interest for experiments currently being undertaken at the ALS (Accélérateur Linéaire de Saclay) and other linear electron accelerator laboratories [2-4]. In that report we derived, starting from the BetheHeitler differential cross section for bremsstrahiung, an approximate expression for the cross section, valid for high energies, $\varepsilon$, of the initial and final electron, and small angles, $\theta$, between intial electron, final electron, and photon. (See fig. 1 and section III for kinematics.) In that approximate expression we neglected terms of relative order $1 / \varepsilon^{2}$ and $\theta^{2}$. Terms of relative order $1 / \varepsilon$ and $\theta$ were retained throughout. We then used this approximate expression to examine in detail the cross section in the region of particular interest for experiments utilizing tagged photons, viz., the region of very small momentum transfers, of the order of the minimum allowed momentum transfer. We saw that although in general the differential cross section was very large in this region, there was a sharp dip in the cross section corresponding to momentum transfers very nearly in the direction of the emitted photon $\left(q_{\perp} \ll q_{z}\right)$. (See fig. 2(a)).

In the present report we extend our investigation of the differential cross section by examining the polarization of tagged photons emitted by a beam of unpolarized electrons. Paralleling our recent report, we now start from the differential Born approximation

$I_{\text {Figures in brackets indicate literature references at the end of this }}$ paper. 
cross section for polarized bremsstrahlung. We write this cross section in section IV, eqs (IV.la) and (IV.Ib), in terms of the polarization vector, $\underline{e}$, of the photon, in a form that is quite similar to that of the Bethe-Heitler cross section. Again paralleling [1], we next write this cross section in a form which explicitely takes account of the large cancellations which occur for small momentum transfers in the expression for the cross section as it is generally written. This is done without making any high energy or small angle approximations, and is presented in eq (IV.5). For the consideration of the polarization we then write the cross section in the form

$$
\begin{aligned}
\frac{d^{3} \sigma}{d \Omega_{p_{2}} d \Omega_{k} d k} & =z^{2} \frac{e^{2}}{\hbar c}\left(\frac{e^{2}}{m c^{2}}\right)^{2} \frac{[1-F(q)]^{2}}{q^{4}}-\frac{1}{k} \frac{p_{2}}{p_{1}} \frac{1}{(2 \pi)^{2}} \\
& x\left\{A \cos ^{2} \psi+2 B \cos \psi \sin \psi+C \sin ^{2} \psi\right\} .
\end{aligned}
$$

(See eqs (IV.la), (IV.5), and (IV.6).) Here $\psi$ is the angle between the photon polarization vector $\underline{e}$ and the production plane (defined by the momentum of the initial electron, $p_{7}$, and the photon momentum, k). The quantities $A, B$, and $C$, which depend on the energies and angles of the electrons and photon, are given by eq (IV.11). The cross sections $d \sigma_{1}$ and $d \sigma_{\|}$, appearing in the conventional definition of polarization, follow directly from eq (I.1): The cross section do $\sigma_{1}$, for photons whose electric vector is perpendicular to the production plane, is given by eq (I.1) with $\psi=\frac{\pi}{2}, \quad\left(\{\}=\{\}_{1}=\mathrm{C}\right)$. The cross 
section $d \sigma_{\| l}$, for photons whose electric vector is in the production plane, is given by eq (I.1) with $\psi=\pi$ (or 0$),\left(\{\}=\{\}_{\|}=A\right)$. The polarization is discussed in section II, and in figs. $3(a)-3(h)$ we plot the polarization as a function of the angle $\theta_{2}$ between the final electron and photon momenta for various values of the azimuthal angle $\phi$, defined in fig. 1, and for two different values of the photon energy, in the region of small momentum transfers.

In section $V$ we derive an approximate expression for the cross section given in eq (I.1), i.e., for the quantities $A, B$, and $C$, valid for high energies and small angles, the neglected terms being of $0\left(1 / \varepsilon^{2}\right)$ and $O\left(\theta^{2}\right)$. Once again, terms of $O(1 / \varepsilon)$ and $O(\theta)$ are retained throughout. These are given in eqs (V.21), (V.22), and (V.25). We show explicitely for the high energy small angle expressions, that $A \geqq 0$, $C \geqq 0$, and $A C-B^{2} \geqq 0$, which insures that eq (I.1) is positive. From eqs (V.22) and (V.25) we then write the numerators appearing in the cross sections $d \sigma_{\perp}$ and $d \sigma_{\|}$in a form similar to that given for the numerator of the cross section for unpolarized photons in eq (VI.49) on p. 66 of [1]. These are given in eqs (V.37) and (V.40). We then have our high energy small angle approximations for the cross sections $d \sigma_{\perp}$ and $d \sigma_{\|}$, which have a form quite similar to that of the cross section for unpolarized photons, given by eq (I.I) on p. 4 of [1]:

$$
\begin{gathered}
d \sigma_{1} \equiv \frac{d^{3} \sigma\left(\psi=\frac{\pi}{2}\right)}{d \Omega_{p_{2}} d \Omega_{k} d k}=z^{2} \frac{e^{2}}{\hbar c}\left(\frac{e^{2}}{m c^{2}}\right)^{2} \frac{[1-F(q)]^{2}}{q^{4}}-\frac{p_{2}}{k} \frac{1}{p_{1}} \frac{1}{(2 \pi)^{2}} \\
\quad x\left\{f_{\perp}(v)(v-u)^{2}+g_{1}(v) \sin ^{2} \frac{1}{2} \phi\right\}
\end{gathered}
$$


and

$$
\begin{aligned}
d \sigma_{\|} \equiv \frac{d^{3} \sigma(\psi=\pi)}{d \Omega_{p_{2}} d \Omega_{k} d k} & =z^{2} \frac{e^{2}}{\hbar c}\left(\frac{e^{2}}{m c^{2}}\right)^{2} \frac{[1-F(q)]^{2}}{q^{4}}-\frac{1}{k} \frac{p_{2}}{p_{1}} \frac{1}{(2 \pi)^{2}} \\
& \times\left\{f_{\|}(v)\left(v-u+\sigma_{\|}\right)^{2}+g_{\|}(v) \sin ^{2} \frac{1 / 2}{2}+\rho_{\|}\right\} .
\end{aligned}
$$

The functions $f_{1}, g_{1}, f_{\|}, g_{\|}, \sigma_{\|}$, and $\rho_{\|}$are defined in eqs (V.39)(V.44). All other variables are defined in section III. In section VI we use eqs (I.2) and (I.3) to obtain analytic expressions for the maxima and minimum of each of these cross sections in the region of small momentum transfers, following closely the detailed analysis given in section VII of [1]. We find that although the cross sections $d \sigma_{1}$ and $d \sigma_{\|}$each exhibit a sharp dip in the same region as that found for the cross section for unpolarized photons in [1] $\left(q_{1} \ll q_{z}\right)$, the minima occur for slightly different values of the angle $\theta_{2}$. (See figs. $2(b)$ and $2(c)$. ) The polarization therefore varies rapidiy in the region of the dip, as shown in figs. $3(a)-3(h)$. Furthermore, the behavior of $d \sigma_{\perp}$ and $d \sigma_{\|}$in the region of the dip, as a function of the azimuthal angle $\phi$, is quite different over most of the photon spectrum (that region given by condition (VI.33)). The cross section $d \sigma_{1}$ behaves similarly to the cross section do for unpolarized photons (discussed in [1]), in that as $\phi$ increases, the sharp dip vanishes, the minimum fuses with the second maximum, and the cross section then has only a single maximum. In contrast, the sharp dip in the cross section $d \sigma_{\|}$remains as $\phi$ increases, provided condition (VI.33) is satisfied. This results in rather large polarization in the region of the dip, as shown in figs. $3(a)-3(h)$. 
Although the point was discussed in detail in [1], we stress again that we are dealing here with the completely differential cross section; once one integrates over the angles of the final electron, as required for the analysis of earlier experiments [5-9] as well as several current experiments using polarized bremsstrahlung $[10,11]$, the sharp dip considered here and in [l] no longer appears. 


\section{POLARIZATION}

The required expressions for the polarization may all be obtained directly from eq (1.1). Relating this to the conventional definition of polarization, the cross section for photons whose electric vector is perpendicular to the production plane, $d \sigma_{1}$, is given by eq (I.1) with $\psi=\frac{\pi}{2}$. The cross section for photons whose electric vector is in the production plane, $d \sigma_{\|}$, is given by eq (I.1) with $\psi=\pi$. The polarization, $P$, is then defined by

$$
\begin{aligned}
P & =\frac{d \sigma_{1}-d \sigma_{\|}}{d \sigma_{1}+d \sigma_{\|}} \\
& =\frac{C-A}{C+A} .
\end{aligned}
$$

In the expression (II.7) for the polarization, and in figs. 3(a)-3(h) giving the polarization as a function of $\theta_{2}$, it is assumed that the directions of the incident beam and the emitted photon are defined with infinite precision, and thus that the production plane is perfectly defined. In an actual experiment, however, the incident beam has a finite angular spread and the photon detector has a finite size. The observed polarization will then depend on the details of the experimental set-up. Although we will not enter into these considerations in this report, it should be noted that they are undoubtedly of importance for those angles for which the polarization varies very rapidly (see figs. $3(a)-3(h)$ ). If, however, it were possible to perform a coincidence measurement which was essentially differential in the angles of the final electron as well as the emitted photon, then we 
could define a polarization referred to two axes which are orthogonal, but arbitrarily oriented with regard to the plane of scattering:

$$
P_{\psi}=\frac{d \sigma_{\psi}-d \sigma_{\psi+\frac{1}{2} \pi}}{d \sigma_{\psi}+d \sigma_{\psi+\frac{1}{2} \pi}},
$$

where $\mathrm{do}_{\psi}$ is the cross section given by eq (I.1), and $d \sigma_{\psi+\frac{1}{2} \pi}$ is this same cross section with $\psi$ replaced by $\psi+\frac{1}{2} \pi$. The polarization, $P$, defined in eq (II.1) is then

$$
P=P_{\frac{1}{2} \pi} \text {. }
$$

Then from eq (I.1)

$$
\begin{aligned}
P_{\psi} & =\frac{\left[A \cos ^{2} \psi+2 B \cos \psi \sin \psi+C \sin ^{2} \psi\right]-\left[A \sin ^{2} \psi-2 B \sin \psi \cos \psi+C \cos ^{2} \psi\right]}{\left[A \cos ^{2} \psi+2 B \cos \psi \sin \psi+C \sin ^{2} \psi\right]+\left[A \sin ^{2} \psi-2 B \sin \psi \cos \psi+C \cos ^{2} \psi\right]} \\
& =\frac{(A-C) \cos 2 \psi+2 B \sin 2 \psi}{A+C}
\end{aligned}
$$$$
=\frac{\sqrt{(A-C)^{2}+4 B^{2}}}{A+C} \cos 2\left(\psi-\psi_{0}\right)
$$

where

$$
A-C=\sqrt{(A-C)^{2}+4 B^{2}} \cos 2 \psi_{0}
$$




$$
\begin{gathered}
2 B=\sqrt{(A-C)^{2}+4 B^{2}} \sin 2 \psi_{0} \\
\tan 2 \psi_{0}=\frac{2 B}{A-C} .
\end{gathered}
$$

Thus if we choose $\psi=\psi_{0}$ or $\psi=\psi_{0}+\frac{1}{2} \pi$ then we have

$$
\begin{aligned}
\left|P_{\psi_{0}}\right| & =\frac{\sqrt{(A-C)^{2}+4 B^{2}}}{A+C} \\
& =\sqrt{\frac{(A+C)^{2}-4\left(A C-B^{2}\right)}{(A+C)^{2}}} \\
& =\sqrt{1-\frac{4\left(A C-B^{2}\right)}{(A+C)^{2}}} .
\end{aligned}
$$

This is the maximum polarization that can be obtained for specified energies and momenta of the final electron and photon. We note from eqs (II.2) and (II.9) that

$$
|P| \leqq\left|P_{\psi_{0}}\right| \leqq 1
$$

In figs. 3(a)-3(h) we plot $P$ as a function of the angle $\theta_{2}$ between the final electron and photon momenta for various values of the azimuthal angle $\phi$, defined in fig. 1 , in the region of small momentum transfers. 


\section{KINEMATICS}

In this section we present the details pertinent to the kinematics and define the various quantities which are used throughout this report.

Unless specified otherwise, we take energies to be in units of the electron rest energy, $\mathrm{mc}^{2}$, and momenta in units of $\mathrm{mc}$. We have

$\varepsilon_{1}, \underline{p}_{1} \quad$ : Energy and momentum of the incident electron

$$
\text { (or positron) } \quad\left(\varepsilon_{1}^{2}-\mathrm{p}_{1}^{2}=1\right) \text {. }
$$

$\varepsilon_{2}, \underline{p}_{2} \quad$ : Energy and momentum of the final electron

$$
\text { (or positron) } \quad\left(\varepsilon_{2}^{2}-\mathrm{p}_{2}{ }^{2}=1\right) \text {. }
$$

$k, \quad$ : Energy and momentum of the emitted photon.

$q=p_{1}-p_{2}-\underline{k}:$ Momentum transferred to the target nucleus.

The energy transferred to the nucleus, which is in general $q_{0}=\varepsilon_{1}-\varepsilon_{2}-k$, is taken throughout this report to be zero, as in the Bethe-Heitier cross section. This is equivalent to the assumption of an infinitely heavy target nucleus. We thereby neglect the effects of the recoil of the target nucleus, both kinematic and dynamic (this latter due to photon emission by the nucleus). For high energies and small angles (in which case $q \lesssim O(u))$, these effects are completely negligible, since they give contributions of relative order $q Z(m / M)$ in the region of the dip, and less elsewhere, $M$ being the mass of the target nucleus.

In the system with $z$-axis in the direction of $\underline{k}$, the angles of $\mathrm{p}_{1}$ are $\left(\theta_{1}, \phi_{7}\right)$, the angles of $\mathrm{p}_{2}$ are $\left(\theta_{2}, \phi_{2}\right)$. These vectors are 
shown in fig. 1 . The components of $p_{1}$ and $\underline{p}_{2}$ perpendicular to $\underline{k}$ are then

$$
\begin{aligned}
& \underline{\mathrm{u}}=\mathrm{p}_{71} \\
& \underline{\mathrm{v}}=\mathrm{p}_{21}
\end{aligned}
$$

The angle between the vectors $\underline{u}$ and $\underline{v}$, which lie in the plane perpendicular to $\underline{k}$, is then

$$
\phi=\phi_{2}-\phi_{1}
$$

The component of $q$ perpendicular to $\underline{k}$ is

$$
\underline{q}_{1}=\underline{u}-\underline{v} .
$$

We have then

$$
q_{1}^{2}=u^{2}-2 u v \cos \phi+v^{2} .
$$

The component of $q$ in the direction of $\underline{k}$ is

$$
q_{z}=p_{1} \cos \theta_{1}-p_{2} \cos \theta_{2}-k \text {. }
$$

The magnitude of the vectors $\underline{u}$ and $\underline{v}$ is given by 


$$
\begin{aligned}
& u=|\underline{u}|=p_{1} \sin \theta_{1} \\
& v=|\underline{v}|=p_{2} \sin \theta_{2} .
\end{aligned}
$$

Throughout the analysis given in this report we use the coordinate system with z-axis in the direction of $\underline{k}$. 
IV. BORN APPROXIMATION DIFFERENTIAL CROSS SECTION FOR POLARIZED PHOTONS EMITTED BY UNPOLARIZED ELECTRONS

The differential Born approximation cross section for polarized photons emitted by unpolarized electrons was first given by May [12] and by Gluckstern, Hull, and Breit [13]. However, we choose to write this cross section in terms of the polarization vector $\underline{e}$ of the photon. Its form is then similar to that of the Bethe-Heitler cross section, and the fact that the sum over polarization states gives the Bethe-Heitler cross section may then be seen by inspection (compare eq (IV.1) on p. 22 of [I]):

$$
d^{3} \sigma=z^{2} \frac{e^{2}}{h c}\left(\frac{e^{2}}{m c^{2}}\right)^{2} \frac{[1-F(q)]^{2}}{q^{4}} \frac{d k}{k} \frac{p_{2}}{p_{1}} \frac{d \Omega_{k} d \Omega_{p_{2}}}{(2 \pi)^{2}}\{\}
$$

where

$$
\begin{aligned}
l\} & =\frac{\left(\underline{p}_{1} \cdot \underline{e}\right)^{2}\left(4 \varepsilon_{2}^{2}-q^{2}\right)}{\left(\varepsilon_{1}-p_{1} \cos \theta_{1}\right)^{2}}+\frac{\left(\underline{p}_{2} \cdot \underline{e}\right)^{2}\left(4 \varepsilon_{1}^{2}-q^{2}\right)}{\left(\varepsilon_{2}-p_{2} \cos \theta_{2}\right)^{2}} \\
& -\frac{2 \underline{p}_{1} \cdot \underline{e} \underline{p}_{2} \cdot \underline{e}\left(4 \varepsilon_{1} \varepsilon_{2}-q^{2}\right)}{\left(\varepsilon_{1}-p_{1} \cos \theta_{1}\right)\left(\varepsilon_{2}-p_{2} \cos \theta_{2}\right)} \\
& +\frac{k^{2}\left(p_{1}^{2} \sin ^{2} \theta_{1}+p_{2}^{2} \sin ^{2} \theta_{2}-2 p_{1} p_{2} \sin \theta_{1} \sin \theta_{2} \cos \phi\right)}{\left(\varepsilon_{1}-p_{1} \cos \theta_{1}\right)\left(\varepsilon_{2}-p_{2} \cos \theta_{2}\right)} .
\end{aligned}
$$


We note here that the first three terms depend on the photon polarization but that the last term does not. This last term therefore differs from the corresponding term in the Bethe-Heitler cross section (eq (IV.1) on p. 22 of [1]) by a factor 2, which appears only after summing over polarization states. Further, and more significantly, we note that the first Born approximation cross section for photons emitted by unpolarized electrons does not have any terms correlating the momenta of the particles with the circular polarization of the photon (of the form ${ }^{2}$ (i $\left.\underline{e} \times \underline{e}^{*}\right) \cdot(\underline{u} \times \underline{v})$, where $\underline{u}$ and $\underline{v}$ are the components of $\underline{p}_{j}$ and $\underline{\mathrm{p}}_{2}$ perpendicular to the photon momentum, $\underline{\mathrm{k}}$ ). Thus in first Born approximation we have only linearly polarized photons. This result follows from the invariance of the cross section for electromagnetic interactions under both space and time reversal, as has been discussed in detail by Kolbenstvedt and 01 sen [14] and 01 sen and Maximon [15]. Although in principal one can have a correlation between the circular polarization of the photon and the momenta of the particles in higher order Born approximation, this effect vanishes for bremsstrahlung at high energies and small angles, [16]; i.e., if we neglect terms of $O(1 / \varepsilon)$ and $O(\theta)$. The circular polarization of photons emitted by unpolarized high energy electrons at small angles is thus negligible. Only in the case of pair production can one produce strongly polarized particles from an initially unpolarized particle at high energies [17]. We return now to the expression appearing in the numerator of the cross section, \{\} as given in eq (IV.Ib), and, in analogy with section VI of [1], write it in the form

2 Here $\underline{e}=\underline{e}_{+}=\frac{1}{\sqrt{2}}(\hat{x}+i \hat{y})$. 


$$
\begin{aligned}
\{\} & =\left(\frac{2 \varepsilon_{2} \underline{u} \cdot \underline{e}}{d_{1}}-\frac{2 \varepsilon_{1} \underline{v} \cdot \underline{e}}{d_{2}}\right)^{2} \\
& -q^{2}\left(\frac{\underline{\underline{u} \cdot \underline{e}}}{d_{1}}-\frac{\underline{v} \cdot \underline{\underline{e}}}{d_{2}}\right)^{2} \\
& +k^{2} \frac{\underline{(\underline{u}-\underline{v})^{2}}}{d_{1} d_{2}}
\end{aligned}
$$

where

$$
\begin{aligned}
& d_{1}=\varepsilon_{1}-p_{1} \cos \theta_{1} \\
& d_{2}=\varepsilon_{2}-p_{2} \cos \theta_{2} .
\end{aligned}
$$

Again we define

$$
\xi=\frac{1}{2 \varepsilon_{1} d_{1}}, \quad \eta=\frac{1}{2 \varepsilon_{2} d_{2}} .
$$

The expression for \{\} may now be written very simply in terms of $\xi$ and $\eta$ :

$$
\begin{aligned}
\{\} & =4 k^{2} \varepsilon_{1} \varepsilon_{2}(\underline{u}-\underline{v})^{2} \xi \eta+16 \varepsilon_{1}{ }^{2} \varepsilon_{2}{ }^{2}(\underline{u} \cdot \underline{e} \xi-\underline{v} \cdot \underline{e} n)^{2} \\
& -4 q^{2}\left(\varepsilon_{1} \underline{u} \cdot \underline{e} \xi-\varepsilon_{2} \underline{v} \cdot \underline{e} \eta\right)^{2}
\end{aligned}
$$


It is worth noting from this expression that for $\underline{u}=\underline{v} \quad(u=v$ and $\phi=0$ ), i.e., for $q_{\perp}=0$, we can write

$$
\{\}=\left[16 \varepsilon_{1}{ }^{2} \varepsilon_{2}{ }^{2}(\xi-\eta)^{2}-4 q^{2}\left(\varepsilon_{1} \xi-\varepsilon_{2} \eta\right)^{2}\right](\underline{u} \cdot \underline{e})^{2} .
$$

The linear polarization, $P$, defined by eq (II.1) is therefore -1 for $q_{\perp}=0$, since then $d \sigma_{\perp}=0$ ( $\left.\underline{e} \perp \underline{u}\right)$. Although the point at which $q_{\perp}=0$ does not correspond exactly to the minimum of the cross section, the dip discussed in [1], it occurs in the region of the dip. We may thus expect strong linear polarization of photons emitted in the region of the dip where $q_{\perp} \ll q_{z}$. (See figs. $3(a)-3(h)$.)

Finally, we note that the expression for \{\} , eq (IV.5), can be written in terms of the polarization angle $\psi$ in the form

$$
\{\}=A \cos ^{2} \psi+2 B \cos \psi \sin \psi+C \sin ^{2} \psi .
$$

Here we have defined the polarization angle $\psi$ by

$$
\underline{u} \cdot \underline{e}=u \cos \psi \quad(|\underline{e}|=1),
$$

i.e., we measure the angle of the polarization vector $\underline{e}$, which lies in the plane perpendicular to $\underline{k}$, from the plane defined by $p_{7}$ and k (see fig. 1).

Then

$$
\underline{v} \cdot \underline{e}=v \cos (\psi-\phi) .
$$


The expressions for \{\} appearing in eqs (1.2) and (I.3) in the numerator of the cross sections $d \sigma_{1}$ (for which $\psi=\frac{\pi}{2}$ ) and $d \sigma_{\|}$ for which $\psi=\pi$ ) follow at once from eq (IV.6):

For $d \sigma_{1}$

$$
\{\}=\{\}_{1}=C \text {. }
$$

For $\mathrm{do}_{\|}$

$$
\{\}=\{\}_{\|}=A \text {. }
$$

Proceeding now to the explicit evaluation of the coefficients A, B, and C in eq (IV.6), we substitute eqs (IV.7) and (IV.8) in eq (IV.5), which gives

$$
\begin{aligned}
\underline{u} \cdot \underline{e} \xi-\underline{v} \cdot \underline{e} n & =u \xi \cos \psi-v n \cos (\psi-\phi) \\
& =(u \xi-v n \cos \phi) \cos \psi-v n \sin \phi \sin \psi
\end{aligned}
$$

and

$$
\varepsilon_{1} \underline{u} \cdot \underline{e} \xi-\varepsilon_{2} \underline{v} \cdot \underline{e} n=\left(\varepsilon_{1} u \xi-\varepsilon_{2} v n \cos \phi\right) \cos \psi-\varepsilon_{2} v n \sin \phi \sin \psi .
$$

Thus, multiplying the first term in eq (IV.5) by $\left(\cos ^{2} \psi+\sin ^{2} \psi\right)$, 


$$
\begin{aligned}
\{\} & =4 k^{2} \varepsilon_{1} \varepsilon_{2}(\underline{u}-\underline{v})^{2} \xi n\left(\cos ^{2} \psi+\sin ^{2} \psi\right) \\
& +16 \varepsilon_{1}{ }^{2} \varepsilon_{2}{ }^{2}[(u \xi-v n \cos \phi) \cos \psi-v n \sin \phi \sin \psi]^{2} \\
& -4 q^{2}\left[\left(\varepsilon_{1} u \xi-\varepsilon_{2} u n \cos \phi\right) \cos \psi-\varepsilon_{2} v n \sin \phi \sin \psi\right]^{2} \\
& =A \cos ^{2} \psi+2 B \cos \psi \sin \psi+C \sin ^{2} \psi
\end{aligned}
$$

where

$$
\begin{aligned}
& A=4 k^{2} \varepsilon_{1} \varepsilon_{2}(\underline{u}-\underline{v})^{2} \xi \eta+16 \varepsilon_{1}{ }^{2} \varepsilon_{2}{ }^{2}(u \xi-v n \cos \phi)^{2}-4 q^{2}\left(\varepsilon_{1} u \xi-\varepsilon_{2} v n \cos \phi\right)^{2} \\
& B=\left[-16 \varepsilon_{1}^{2} \varepsilon_{2}^{2}(u \xi-v n \cos \phi) v \eta+4 q^{2}\left(\varepsilon_{1} u \xi-\varepsilon_{2} v n \cos \phi\right) \varepsilon_{2} v n\right] \sin \phi \\
& C=4 k^{2} \varepsilon_{7} \varepsilon_{2}(\underline{u}-\underline{v})^{2} \xi \eta+16 \varepsilon_{1}^{2} \varepsilon_{2}^{2} v^{2} \eta^{2} \sin ^{2} \phi-4 q^{2} \varepsilon_{2}^{2} v^{2} \eta^{2} \sin ^{2} \phi .
\end{aligned}
$$

We note that if one integrates the cross section over the angle $\phi$ of the final electron, then the integral of the term with the factor $B$ is zero. This follows from the fact that this term has the factor $\sin \phi$ and that its only other dependence on $\phi$ (e.g., through $q^{2}$ ) is a function of $\cos \phi$. Thus on integrating over the angles of the final electron we have an expression of the form

$$
\bar{A} \cos ^{2} \psi+\bar{C} \sin ^{2} \psi=\bar{A}+(\bar{C}-\bar{A}) \sin ^{2} \psi
$$

as should be expected [5]. 
In the following section we derive the high energy small angle approximation to \{\} , i.e., we obtain approximations for $A, B$, and $C$ valid for high energies and small angles. 


\section{TO THE DIFFERENTIAL CROSS SECTION FOR POLARIZED PHOTONS}

We now consider the high energy small angle approximation to \{\} as given by eq (IV.5). Noting the similarity of eq (IV.5) to the expression (IV.4) in [1], we may follow step by step the analysis given there with very little modification. For the last term in eq (IV.5) we then find, from pp. 50-54 of [1], terminating in expression (VI.28) of [1], the high energy small angle approximation

$$
-\left(\frac{k^{2} \underline{u} \cdot \underline{e}}{\varepsilon_{1} \varepsilon_{2}}\right)^{2} .
$$

Again following [1], we have, from eq (VI.29) on p. 54 there, the high energy smal1 angle approximation to the first term in eq (IV.5), viz.,

$$
4 k^{2} \varepsilon_{1} \varepsilon_{2}(\underline{u}-\underline{v})^{2} \xi_{0} n_{0}
$$

where

$$
\begin{aligned}
& \xi=\xi_{0}-\xi_{1} \\
& n=n_{0}-n_{1}
\end{aligned}
$$

and 


$$
\begin{array}{ll}
\xi_{0}=\frac{1}{1+u^{2}}, & \xi_{1}=\frac{1}{2 \varepsilon_{1}\left(\varepsilon_{1}+p_{1} \cos \theta_{1}\right)} \approx \frac{1}{4 \varepsilon_{1}^{2}} \\
n_{0}=\frac{1}{1+v^{2}}, & \eta_{1}=\frac{1}{2 \varepsilon_{2}\left(\varepsilon_{2}+p_{2} \cos \theta_{2}\right)} \approx \frac{1}{4 \varepsilon_{2}^{2}} .
\end{array}
$$

Finally, we obtain the high energy, small angle approximation for the second term in eq (IV.5). Substituting eq (V.3) in (IV.5) we have

$$
\begin{aligned}
16 \varepsilon_{1}{ }^{2} \varepsilon_{2}^{2}(\underline{u} \cdot \underline{e} \xi-\underline{v} \cdot \underline{e} n)^{2} & =16 \varepsilon_{1}^{2} \varepsilon_{2}^{2}\left(\underline{u} \cdot \underline{e} \xi_{0}-\underline{v} \cdot \underline{e} n_{0}\right)^{2} \\
& -32 \varepsilon_{1}^{2} \varepsilon_{2}^{2}\left(\underline{u} \cdot \underline{e} \xi_{0}-\underline{u} \cdot \underline{e} n_{0}\right)\left(\underline{u} \cdot \underline{e} \xi_{1}-\underline{v} \cdot \underline{e} n_{1}\right) \\
& +16 \varepsilon_{1}^{2} \varepsilon_{2}^{2}\left(\underline{u} \cdot \underline{e} \xi_{1}-\underline{v} \cdot \underline{e} n_{1}\right)^{2} \\
& \approx 16 \varepsilon_{1}{ }^{2} \varepsilon_{2}^{2}\left(\underline{u} \cdot \underline{e} \xi_{0}-\underline{v} \cdot \underline{e} n_{0}\right)^{2} \\
& +8 k\left(\varepsilon_{1}+\varepsilon_{2}\right)\left(\underline{u} \cdot \underline{e} \xi_{0}-\underline{v} \cdot \underline{e} n_{0}\right)(\underline{u} \cdot \underline{e}) \\
& +\left(\frac{k\left(\varepsilon_{1}+\varepsilon_{2}\right)}{\varepsilon_{1} \varepsilon_{2}}\right)^{2}(\underline{u} \cdot \underline{e})^{2} .
\end{aligned}
$$

Here we have neglected terms of order $1 / \varepsilon^{2}$ and $\theta^{2}$ relative to those retained, as on pp. 54-59 of [1]. The last three lines are, respectively, of $0\left(\varepsilon^{4} \xi^{2} q_{1}^{2}\right), \quad 0\left(\varepsilon^{2} u \xi q_{1}\right)$, and $O\left(u^{2}\right)$, as in eq (VI.20) on p. 48 of [1]. 
We now have, in eqs (V.2), (V.5), and (V.1), the high energy small angle approximations to the first, second, and third terms in eq (IV.5), respectively. We thus have

$$
\begin{aligned}
\{\} & \approx 4 k^{2} \varepsilon_{1} \varepsilon_{2} \underline{(u-\underline{v})^{2} \xi_{0} \eta_{0}} \\
& +16 \varepsilon_{1}{ }^{2} \varepsilon_{2}{ }^{2}\left[\underline{u} \cdot \underline{e} \xi_{0}-\underline{v} \cdot \underline{e} \underline{n}_{0}+\frac{k\left(\varepsilon_{1}+\varepsilon_{2}\right)}{4 \varepsilon_{1}{ }^{2} \varepsilon_{2}{ }^{2}} \underline{u} \cdot \underline{e}\right]^{2} \\
& -\left(\frac{k^{2}}{\varepsilon_{1} \varepsilon_{2}}\right)^{2}(\underline{u} \cdot \underline{e})^{2} .
\end{aligned}
$$

We next write the expression for \{\} in a form that is manifestly positive. This aids later in the analytical determination of the maxima and minima of the polarization as a function of the angles of the final electron and photon. It also eliminates the large cancellations which otherwise arise in the numerical evaluation of the cross section. To this end we write

$$
(\underline{u}-\underline{v})^{2}=(u-v)^{2}+4 u v \sin ^{2} \frac{1}{2} \phi
$$

Substituting eqs (IV.7) and (IV.8) in (V.6) we have 


$$
\begin{aligned}
\underline{u} \cdot \underline{e} \xi_{0}-\underline{v} \cdot \underline{e n}_{0} & =\frac{u}{1+u^{2}} \cos \psi-\frac{v}{1+v^{2}} \cos (\psi-\phi) \\
& =\frac{u}{1+u^{2}} \cos \psi-\frac{v}{1+y^{2}} \cos \psi \cos \phi-\frac{v}{1+v^{2}} \sin \psi \sin \phi \\
& =\frac{u}{1+u^{2}} \cos \psi-\frac{v}{1+y^{2}} \cos \psi+\frac{v}{1+v^{2}}(1-\cos \phi) \cos \psi-\frac{v}{1+v^{2}} \sin \psi \sin \phi,
\end{aligned}
$$

and thus

$$
\begin{aligned}
\underline{u} \cdot \underline{e} \xi_{0}-\underline{v} \cdot \underline{e n}_{0} & =\left(\frac{u}{1+u^{2}}-\frac{v}{1+v^{2}}\right) \cos \psi+\frac{2 v \cos \psi}{1+v^{2}} \sin ^{2} \frac{1}{2} \phi-\frac{2 v}{1+v^{2}} \sin \psi \sin \frac{1}{2} \phi \cos ^{\frac{1}{2}} \phi \\
& =\left((u-v)(1-u v) \xi_{0} n_{0}+2 v n_{0} \sin ^{2} \frac{1}{2} \phi\right) \cos \psi \\
& -v n_{0} \sin \phi \sin \psi
\end{aligned}
$$

Substituting eqs (IV.7), (V.7), and (V.8) in (V.6) we obtain

$$
\begin{aligned}
& \{\} \approx 4 k^{2} \varepsilon_{1} \varepsilon_{2} \xi_{0} n_{0}\left[(u-v)^{2}+4 u v \sin ^{2} 2_{\frac{1}{2}} \phi\right] \\
& +16 \varepsilon_{1}{ }^{2} \varepsilon_{2}{ }^{2}\left[\begin{array}{l}
\left.(u-v)(1-u v) \xi_{0} n_{0}+2 v n_{0} \sin ^{2} \frac{1}{2} \phi+\frac{k\left(\varepsilon_{1}+\varepsilon_{2}\right) u}{4 \varepsilon_{1}{ }^{2} \varepsilon_{2}{ }^{2}}\right) \cos \psi \\
-v n_{0} \sin \phi \sin \psi
\end{array}\right]^{2} \\
& -\left(\frac{k^{2}}{\varepsilon_{7} \varepsilon_{2}}\right)^{2} u^{2} \cos ^{2} \psi \text {. }
\end{aligned}
$$


At this point we note that if this high energy small angle expression for \{\} is summed over the polarization states of the emitted photon (adding its value for a given $\psi$ to its value with $\psi$ replaced by $\psi+\frac{1}{2} \pi$ ) then the result is independent of $\psi$ and is equal to the expression obtained earlier, in [1]. For the first term in eq (V.9) we then obtain

$$
8 k^{2} \varepsilon_{1} \varepsilon_{2} \xi_{0} n_{0}\left[(u-v)^{2}+4 u v \sin ^{2} \frac{1}{2} \phi\right]
$$

which is the term given in [1], p. 54, eq (VI.29). For the last term in eq (V.9) we have

$$
-\left(\frac{k^{2}}{\varepsilon_{1} \varepsilon_{2}}\right)^{2} u^{2} \cos ^{2} \psi-\left(\frac{k^{2}}{\varepsilon_{1} \varepsilon_{2}}\right)^{2} u^{2} \cos ^{2}\left(\psi+\frac{1}{2} \pi\right)=-\left(\frac{k^{2}}{\varepsilon_{1} \varepsilon_{2}}\right)^{2} u^{2},
$$

which is the term given in [1], p. 54 eq (VI.28). Finally, we note that the second term in eq $(V .9)$ is of the form

$$
(a \cos \psi+b \sin \psi)^{2}
$$

Summing over polarization states then gives

$$
\begin{aligned}
(a \cos \psi+b \sin \psi)^{2} & +\left(a \cos \left(\psi+\frac{1}{2} \pi\right)+b \sin \left(\psi+\frac{1}{2} \pi\right)\right)^{2} \\
& =(a \cos \psi+b \sin \psi)^{2}+(-a \sin \psi+b \cos \psi)^{2} \\
& =a^{2}+b^{2} . .
\end{aligned}
$$


Therefore from the second term in eq (V.9) we have

$$
\begin{aligned}
& 16 \varepsilon_{1}{ }^{2} \varepsilon_{2}{ }^{2}\left[\left((u-v)(1-u v) \xi_{0} n_{0}+2 v n_{0} \sin ^{2}{ }_{\frac{1}{2} \phi}+\frac{k\left(\varepsilon_{1}+\varepsilon_{2}\right) u}{4 \varepsilon_{1}{ }^{2} \varepsilon_{2}{ }^{2}}\right)^{2}+v^{2} n_{0}{ }^{2} \sin ^{2} \phi\right] \\
& =16 \varepsilon_{1}{ }^{2} \varepsilon_{2}{ }^{2}\left[(u-v)^{2}(1-u v)^{2} \xi_{0}{ }^{2} n_{0}{ }^{2}+4(u-v)(1-u v) \xi_{0} n_{0} v_{0} \sin ^{2}{ }^{2} \frac{1}{2} \phi\right. \\
& +4 v^{2} n_{0}^{2} \sin ^{4 \frac{1}{2} \phi}+v^{2} n_{0}^{2} \sin ^{2} \phi \\
& +\left((u-v)(1-u v) \xi_{0} n_{0}+2 v n_{0} \sin ^{2} \frac{z_{2}}{2} \phi\right) \frac{2 k\left(\varepsilon_{1}+\varepsilon_{2}\right) u}{4 \varepsilon_{1}{ }^{2} \varepsilon_{2}{ }^{2}} \\
& \left.+\frac{k^{2}\left(\varepsilon_{1}+\varepsilon_{2}\right)^{2} u^{2}}{16 \varepsilon_{1}{ }^{4} \varepsilon_{2}^{4}}\right]
\end{aligned}
$$

In the first term in the square bracket above we may write

$$
\begin{aligned}
(1-u v)^{2} & =1-2 u v+u^{2} v^{2} \\
& =\left(1+u^{2}\right)\left(1+v^{2}\right)-(u+v)^{2}
\end{aligned}
$$

and hence

$$
(u-v)^{2}(1-u v)^{2} \xi_{0}^{2} n_{0}^{2}=(u-v)^{2} \xi_{0} n_{0}-\left(u^{2}-v^{2}\right)^{2} \xi_{0}^{2} n_{0}^{2}
$$

The next three terms in the above square bracket are 


$$
4(u-v)(1-u v) \xi_{0} n_{0} v n_{0} \sin ^{2} \frac{1}{2} \phi+4 v^{2} n_{0}{ }^{2} \sin ^{4} \frac{4_{1}}{2} \phi+v^{2} n_{0}{ }^{2} \sin ^{2} \phi .
$$

The last two terms here combine to give

$$
\begin{aligned}
4 v^{2} n_{0}^{2} \sin ^{4 \frac{1}{2} \phi}+v^{2} n_{0}^{2} \sin ^{2} \phi & =4 v^{2} n_{0}^{2} \sin ^{2} \frac{1}{2} \phi\left(\sin ^{2} \frac{1}{2} \phi+\cos ^{2} \frac{1}{2} \phi\right) \\
& =4 v^{2} n_{0}^{2} \sin ^{2} \frac{1}{\frac{1}{2}} \phi .
\end{aligned}
$$

Adding this to the first term we have

$$
\begin{aligned}
4(u-v)(1-u v) \xi_{0} n_{0} v n_{0} \sin ^{2} \frac{1}{2} \phi & +4 v^{2} n_{0}{ }^{2} \sin ^{2} \frac{1}{\frac{1}{2} \phi} \\
& =4 v n_{0}{ }^{2} \sin ^{2} \frac{1}{2} \phi\left[(u-v)(1-u v) \xi_{0}+v\right] \\
& =4 v \xi_{0} n_{0}{ }^{2} \sin ^{2} \frac{1}{\frac{1}{2}} \phi\left[(u-v)(1-u v)+v\left(1+u^{2}\right)\right] \\
& =4 v \xi_{0} n_{0}{ }^{2} \sin ^{2}{ }^{2 \frac{1}{2}} \phi\left[u\left(1+v^{2}\right)\right] \\
& =4 u v \xi_{0} n_{0} \sin ^{2} \frac{1}{\frac{1}{2} \phi} .
\end{aligned}
$$

Adding this to the expression (V.13) we have, for the first four terms in eq (V.12)

$$
\begin{aligned}
\xi_{0} n_{0}\left[(u-v)^{2}+4 u v \sin ^{2} \frac{1}{2} \phi\right] & -\left(u^{2}-v^{2}\right)^{2} \xi_{0}{ }^{2} n_{0}{ }^{2} \\
& =(\underline{u}-\underline{v})^{2} \xi_{0} n_{0}-\left(u^{2}-v^{2}\right)^{2} \xi_{0}{ }^{2} n_{0}{ }^{2},
\end{aligned}
$$


which is the expression in square brackets in [1], p. 59, eq (VI.38). Next we observe that the last term in eq (V.12) is, together with the factor $16 \varepsilon_{1}{ }^{2} \varepsilon_{2}{ }^{2}$ outside the square bracket,

$$
\left(\frac{k\left(\varepsilon_{1}+\varepsilon_{2}\right)}{\varepsilon_{1} \varepsilon_{2}}\right)^{2} u^{2} \text {, }
$$

which is the last term in [1], p. 59, eq (VI.38).

Finally we have the remaining term in eq (V.12), viz., (including the factor $16 \varepsilon_{1}{ }^{2} \varepsilon_{2}{ }^{2}$ ),

$$
8 k\left(\varepsilon_{1}+\varepsilon_{2}\right) u\left((u-v)(1-u v) \xi_{0} \eta_{0}+2 v n_{0} \sin ^{2}{ }_{1 / 2} \phi\right) .
$$

We note that the term here with factor $\sin ^{2} \frac{1}{2} \phi$ is of $0\left(1 / \varepsilon^{2}\right)$ relative to the term with this same factor in eq (V.14) (when we include the overall factor $16 \varepsilon_{1}{ }^{2} \varepsilon_{2}{ }^{2}$ in this latter term). It may therefore be neglected. We are then left with

$$
8 k\left(\varepsilon_{1}+\varepsilon_{2}\right) u(1-u v) \xi_{0} n_{0}(u-v) \quad .
$$

Here we note that we may, in the factors multiplying (u-v) in eq (v.17), set $v=u$, again neglecting terms of $0\left(1 / \varepsilon^{2}\right)$ relative to terms retained in eq (V.14). Thus we may write

$$
1-u v \approx-\left(u^{2}-1\right)
$$


and

$$
u \approx \frac{(u+v)}{2}
$$

giving, for eq (V.17),

$$
-4 k\left(\varepsilon_{1}+\varepsilon_{2}\right)\left(u^{2}-1\right) \xi_{0} n_{0}\left(u^{2}-v^{2}\right)
$$

which is the second term in [1], p. 59, eq (VI.38).

Our expression (V.9), when summed over any two orthogonal polarization states, thus indeed gives the expression for \{\} derived in [1], with neglect of terms of relative orders $1 / \varepsilon^{2}$ and $\theta^{2}$.

We now return to the task of writing the expression for \{\} in a form that is manifestly positive. It is clear that the original expression for \{\} , eqs (IV.Ta) and (IV.Tb), before making the high energy small angle approximations, can not be negative since the cross section can not be negative. We wish to show, however, that our high energy small angle approximation does not introduce errors such that the expression for \{\} then becomes negative for certain values of the variables. Since this involves rather tedious algebraic manipulation, the reader may wish to skip to p. 44. To this end we note that eq (V.9) may (after multiplying the first term there by $\left(\cos ^{2} \psi+\sin ^{2} \psi\right)$ ) be written in the form

$$
\{\}=A \cos ^{2} \psi+2 B \cos \psi \sin \psi+C \sin ^{2} \psi
$$

where 


$$
\begin{aligned}
& A=4 \varepsilon_{1} \varepsilon_{2} \xi_{0} n_{0}\left[k^{2}+4 \varepsilon_{1} \varepsilon_{2}(1-u v)^{2} \xi_{0} n_{0}\right](u-v)^{2} \\
& +32 \varepsilon_{1}{ }^{2} \varepsilon_{2}{ }^{2}(1-u v) \xi_{0} n_{0}\left[2 v n_{0} \sin ^{2 \frac{1}{2} \phi}+\frac{k\left(\varepsilon_{1}+\varepsilon_{2}\right) u}{4 \varepsilon_{1}{ }^{2} \varepsilon_{2}{ }^{2}}\right](u-v) \\
& +16 \varepsilon_{1}{ }^{2} \varepsilon_{2}{ }^{2}\left[2 v n_{0} \sin ^{2{ }^{\frac{1}{2}} \phi}+\frac{k\left(\varepsilon_{1}+\varepsilon_{2}\right) u}{4 \varepsilon_{1}{ }^{2} \varepsilon_{2}{ }^{2}}\right]^{2} \\
& +4 k^{2} \varepsilon_{7} \varepsilon_{2} \xi_{0} n_{0} \cdot 4 u v \sin ^{2} \frac{i_{2}}{2} \phi \\
& -\left(\frac{k^{2}}{\varepsilon_{1} \varepsilon_{2}}\right)^{2} u^{2}
\end{aligned}
$$

$B=-16 \varepsilon_{1}^{2} \varepsilon_{2}^{2} v n_{0} \sin \phi\left[(u-v)(1-u v) \xi_{0} n_{0}+2 v n_{0} \sin ^{2} \frac{z_{2} \phi}{2}+\frac{k\left(\varepsilon_{1}+\varepsilon_{2}\right) u}{4 \varepsilon_{1}^{2} \varepsilon_{2}^{2}}\right]$,

$c=16 \varepsilon_{1}{ }^{2} \varepsilon_{2}{ }^{2} v^{2} n_{0}{ }^{2} \sin ^{2} \phi+4 k^{2} \varepsilon_{1} \varepsilon_{2} \xi_{0} n_{0}\left[(u-v)^{2}+4 u v \sin ^{2}{ }^{2} \frac{1}{2} \phi\right]$.

Clearly $C \geqq 0$. Let us next show that $A \geqq 0$. Completing the square in eq (V.20) we have 


$$
\begin{aligned}
& \left.A=4 \varepsilon_{1} \varepsilon_{2} \xi_{0} n_{0}\left[k^{2}+4 \varepsilon_{1} \varepsilon_{2}(1-u v)\right]^{2} \xi_{0} n_{0}\right] \\
& x\left[u-v+\frac{4 \varepsilon_{1} \varepsilon_{2}(1-u v)\left(2 v \eta_{0} \sin ^{2}{ }^{2} \frac{1}{2} \phi+\frac{k\left(\varepsilon_{1}+\varepsilon_{2}\right) u}{4 \varepsilon_{1}{ }^{2} \varepsilon_{2}{ }^{2}}\right)}{k^{2}+4 \varepsilon_{1} \varepsilon_{2}(7-u v)^{2} \xi_{0} n_{0}}\right]^{2} \\
& -\frac{4 \varepsilon_{1} \varepsilon_{2} \xi_{0} n_{0}\left[4 \varepsilon_{1} \varepsilon_{2}(1-u v)\right]^{2}\left[2 v n_{0} \sin ^{2 \frac{1}{2} \phi}+\frac{k\left(\varepsilon_{1}+\varepsilon_{2}\right) u}{4 \varepsilon_{1}{ }^{2} \varepsilon_{2}{ }^{2}}\right]^{2}}{k^{2}+4 \varepsilon_{1} \varepsilon_{2}(1-u v)^{2} \xi_{0} n_{0}} \\
& +16 \varepsilon_{1}{ }^{2} \varepsilon_{2}{ }^{2}\left[2 v \eta_{0} \sin ^{2}{ }^{2}{ }_{2} \phi+\frac{k\left(\varepsilon_{1}+\varepsilon_{2}\right) u}{4 \varepsilon_{1}{ }^{2} \varepsilon_{2}{ }^{2}}\right]^{2} \\
& +16 k^{2} \varepsilon_{1} \varepsilon_{2} \xi_{0} n_{0} u v \sin ^{2} \frac{1}{2} \phi-\left(\frac{k^{2}}{\varepsilon_{1} \varepsilon_{2}}\right)^{2} u^{2} .
\end{aligned}
$$

Adding the second and third terms in eq (V.23) we have 


$$
\begin{aligned}
& \frac{16 \varepsilon_{1}{ }^{2} \varepsilon_{2}{ }^{2}\left[2 v n_{0} \sin ^{2}{ }^{2} \frac{1 / 2}{2} \phi+\frac{k\left(\varepsilon_{1}+\varepsilon_{2}\right) u}{4 \varepsilon_{1}^{2} \varepsilon_{2}^{2}}\right]^{2}}{k^{2}+4 \varepsilon_{1} \varepsilon_{2}(1-u v)^{2} \xi_{0} n_{0}}\left[\begin{array}{l}
k^{2}+4 \varepsilon_{1} \varepsilon_{2}(1-u v)^{2} \xi_{0} n_{0} \\
-4 \varepsilon_{1} \varepsilon_{2}(1-u v)^{2} \xi_{0} n_{0}
\end{array}\right] \\
& =\frac{16 k^{2} \varepsilon_{1}{ }^{2} \varepsilon_{2}{ }^{2}\left[2 v_{0} \sin ^{2{ }^{2}{ }_{2} \phi}+\frac{k\left(\varepsilon_{1}+\varepsilon_{2}\right) u}{4 \varepsilon_{1}{ }^{2} \varepsilon_{2}{ }^{2}}\right]^{2}}{k^{2}+4 \varepsilon_{1} \varepsilon_{2}(1-u v)^{2} \xi_{0} n_{0}} .
\end{aligned}
$$

We now have

$$
\begin{aligned}
A & \left.=4 \varepsilon_{1} \varepsilon_{2} \xi_{0} n_{0}\left[k^{2}+4 \varepsilon_{1} \varepsilon_{2}(1-u v)\right]^{2} \xi_{0} n_{0}\right] \\
& \left.x\left[u-v+\frac{4 \varepsilon_{1} \varepsilon_{2}(1-u v)\left[2 v n_{0} \sin ^{2}{ }_{\frac{1}{2} \phi}+\frac{k\left(\varepsilon_{1}+\varepsilon_{2}\right) u}{4 \varepsilon_{1}{ }^{2} \varepsilon_{2}^{2}}\right]}{k^{2}+4 \varepsilon_{1} \varepsilon_{2}(1-u v)^{2} \xi_{0} n_{0}}\right]\right]^{2} \\
& +\frac{16 k^{2} \varepsilon_{1}{ }^{2} \varepsilon_{2}{ }^{2}\left[2 v n_{0} \sin ^{2}{ }^{2} \frac{1}{2} \phi+\frac{k\left(\varepsilon_{1}+\varepsilon_{2}\right) u}{4 \varepsilon_{1}{ }^{2} \varepsilon_{2}{ }^{2}}\right]^{2}}{k^{2}+4 \varepsilon_{1} \varepsilon_{2}(1-u v)^{2} \xi_{0} n_{0}} \\
& +16 k^{2} \varepsilon_{1} \varepsilon_{2} \xi_{0} n_{0} u v \sin ^{2}{ }^{2} \frac{1}{2} \phi-\left(\frac{k^{2}}{\varepsilon_{1} \varepsilon_{2}}\right)^{2} u^{2} .
\end{aligned}
$$


Here we add the second and fourth terms and obtain

$$
\begin{aligned}
& \frac{16 k^{2} \varepsilon_{1}{ }^{2} \varepsilon_{2}{ }^{2}\left[2 v \eta_{0} \sin ^{2}{ }^{2} \frac{k}{2} \phi+\frac{k\left(\varepsilon_{1}+\varepsilon_{2}\right) u}{4 \varepsilon_{1}{ }^{2} \varepsilon_{2}{ }^{2}}\right]^{2}}{k^{2}+4 \varepsilon_{1} \varepsilon_{2}(1-u v)^{2} \xi_{0} \eta_{0}}-\left(\frac{k^{2}}{\varepsilon_{1} \varepsilon_{2}}\right)^{2} u^{2} \\
& =\frac{16 k^{2} \varepsilon_{1}{ }^{2} \varepsilon_{2}{ }^{2}\left[4 v^{2} n_{0}{ }^{2} \sin ^{4} \phi+\frac{k\left(\varepsilon_{1}+\varepsilon_{2}\right) u}{\varepsilon_{1}{ }^{2} \varepsilon_{2}{ }^{2}} v_{0} \sin ^{2}{ }^{2} \frac{1}{2} \phi\right]+\frac{k^{4}\left(\varepsilon_{1}+\varepsilon_{2}\right)^{2} u^{2}}{\varepsilon_{1}{ }^{2} \varepsilon_{2}{ }^{2}}}{k^{2}+4 \varepsilon_{1} \varepsilon_{2}(T-u v)^{2} \xi_{0} n_{0}} \\
& -\left[\frac{\frac{k^{6} u^{2}}{\varepsilon_{1}^{2} \varepsilon_{2}^{2}}+4 \varepsilon_{1} \varepsilon_{2}(1-u v)^{2} \xi_{0} n_{0}\left(\frac{k^{2}}{\varepsilon_{1} \varepsilon_{2}}\right)^{2} u^{2}}{k^{2}+4 \varepsilon_{1} \varepsilon_{2}(1-u v)^{2} \xi_{0} n_{0}}\right] \\
& =\frac{1}{\left[k^{2}+4 \varepsilon_{1} \varepsilon_{2}(1-u v)^{2} \xi_{0} n_{0}\right]}
\end{aligned}
$$

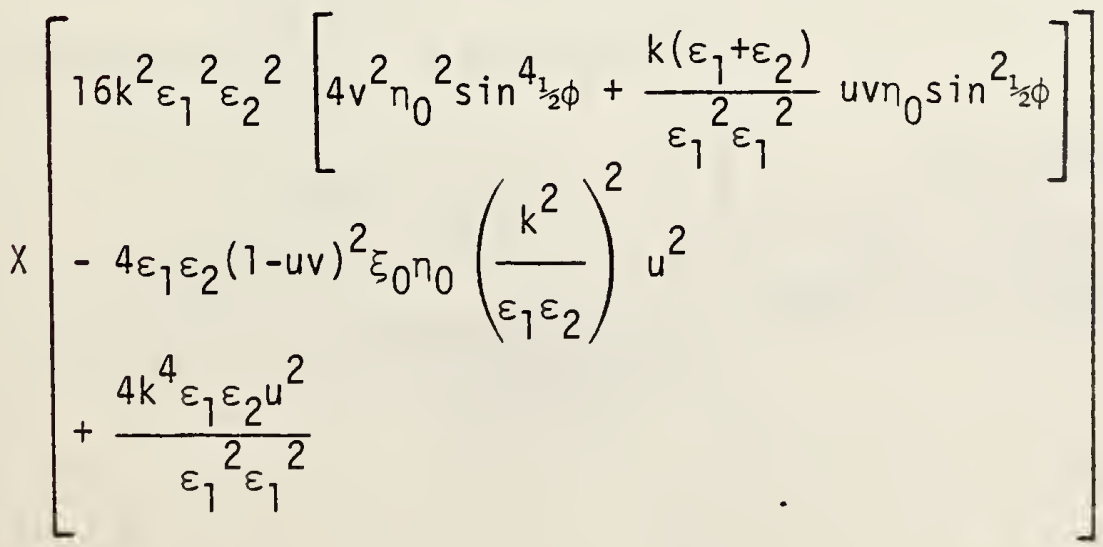

31 
The last two terms in the last square bracket in the last expression are

$$
\begin{aligned}
\frac{4 k^{4} u^{2}}{\varepsilon_{1} \varepsilon_{2}}\left[1-\frac{(1-u v)^{2}}{\left(1+u^{2}\right)\left(1+v^{2}\right)}\right] & =\frac{4 k^{4} u^{2}}{\varepsilon_{1} \varepsilon_{2}} \xi_{0} n_{0}\left[\left(1+u^{2}\right)\left(1+v^{2}\right)-(1-u v)^{2}\right] \\
& =\frac{4 k^{4} u^{2} \xi_{0} n_{0}(u+v)^{2}}{\varepsilon_{1} \varepsilon_{2}} .
\end{aligned}
$$

Thus we have

$$
\begin{aligned}
& A=4 \varepsilon_{1} \varepsilon_{2} \xi_{0} \eta_{0}\left[k^{2}+4 \varepsilon_{1} \varepsilon_{2}(1-u v)^{2} \xi_{0} n_{0}\right] \\
& x\left[u-v+\frac{4 \varepsilon_{7} \varepsilon_{2}(1-u v)\left[2 v n_{0} \sin ^{2} \frac{1}{2} \phi+\frac{k\left(\varepsilon_{1}+\varepsilon_{2}\right) u}{4 \varepsilon_{1}^{2} \varepsilon_{2}^{2}}\right]}{k^{2}+4 \varepsilon_{1} \varepsilon_{2}(1-u v)^{2} \xi_{0} n_{0}}\right]^{2} \\
& +16 k^{2} \varepsilon_{1} \varepsilon_{2} \xi_{0} n_{0} \text { uvs in }{ }^{2} \frac{1}{2} \phi \\
& +\frac{16 k^{2} \varepsilon_{1}^{2} \varepsilon_{2}^{2}}{\left[k^{2}+4 \varepsilon_{1} \varepsilon_{2}(1-u v)^{2} \xi_{0} n_{0}\right]}
\end{aligned}
$$

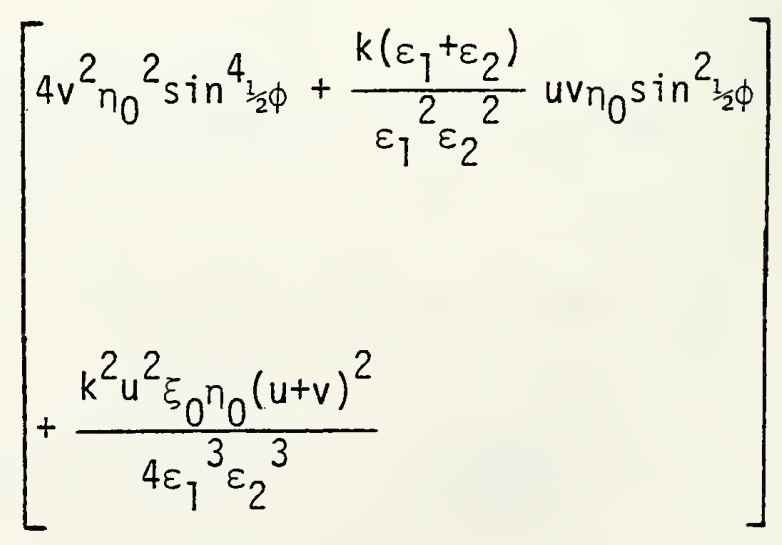


This expression may be simplified somewhat. In the last square bracket in eq $(V .24)$ the term with factor $\sin ^{2} \frac{1}{2} \phi$ gives a contribution to $A$ of order $1 / \varepsilon^{2}$ relative to the second term in eq $(V .24)$, which also has a factor $\sin ^{2} \frac{1}{2} \phi$. It may therefore be neglected. In this same square bracket the last term gives a contribution to $A$ of $0\left(u^{2}\right)$. It is therefore of $0\left(\phi^{2} / \varepsilon^{4}\right)$ relative to the second term in eq (V.24). It need be retained, therefore, only if $\phi \leqslant 0\left(1 / \varepsilon^{2}\right)$. But in that case it is negligible relative to the first term in eq (V.24), unless $u-v \lesssim 0\left(u / \varepsilon^{2}\right)$. We may therefore set $v=u$ and $n_{0}=\xi_{0}$ in the last term in eq (V.24). We then obtain

$$
\begin{aligned}
A & =4 \varepsilon_{1} \varepsilon_{2} \xi_{0} \eta_{0}\left[k^{2}+4 \varepsilon_{1} \varepsilon_{2}(1-u v)^{2} \xi_{0} \eta_{0}\right] \\
& x\left[u-v+\frac{4 \varepsilon_{1} \varepsilon_{2}(1-u v)\left[2 v \eta_{0} \sin ^{2 \frac{1}{2} \phi}+\frac{k\left(\varepsilon_{1}+\varepsilon_{2}\right) u}{4 \varepsilon_{1}{ }^{2} \varepsilon_{2}{ }^{2}}\right]}{k^{2}+4 \varepsilon_{1} \varepsilon_{2}(1-u v)^{2} \xi_{0} n_{0}}\right]^{2} \\
& +16 k^{2} \varepsilon_{1} \varepsilon_{2} \xi_{0} n_{0} u v \sin ^{2} \frac{1}{2} \phi \\
& +\frac{16 k^{2} \varepsilon_{1}{ }^{2} \varepsilon_{2}{ }^{2}}{\left[k^{2}+4 \varepsilon_{1} \varepsilon_{2}(1-u v)^{2} \xi_{0} \eta_{0}\right]}\left[4 v^{2} n_{0}{ }^{2} \sin ^{4_{\frac{1}{2}} \phi}+\frac{k^{2} \xi_{0}{ }^{2} u^{4}}{\varepsilon_{1}{ }^{3} \varepsilon_{2}{ }^{3}}\right] .
\end{aligned}
$$


Thus we also have $A \geqq 0$. We may now write eq (V.19) in the form

$$
=\frac{1}{A}\left[(A \cos \psi+B \sin \psi)^{2}+\left(A C-B^{2}\right) \sin ^{2} \psi\right],
$$

from which we see that the expression for \{\} will be positive provided that

$$
A C-B^{2} \geqq 0
$$

We therefore return to the expressions $(V .20)-(V .22)$ for $A, B$, and $C$, and show directly that $A C-B^{2} \geqq 0$. From eq (V.22) we may write

$$
\begin{aligned}
A C-B^{2} & =4 k^{2} \varepsilon_{1} \varepsilon_{2} \xi_{0} n_{0}\left[(u-v)^{2}+4 u v \sin ^{2} \frac{1}{2} \phi\right] \cdot A \\
& +16 \varepsilon_{1}{ }^{2} \varepsilon_{2}{ }^{2} v^{2} n_{0}{ }^{2} \sin ^{2} \phi \cdot A-B^{2},
\end{aligned}
$$

and from eqs (V.20) and (V.21)

$$
\begin{aligned}
A & =4 k^{2} \varepsilon_{1} \varepsilon_{2} \xi_{0} n_{0}\left[(u-v)^{2}+4 u v \sin ^{2} \frac{1}{2} \phi\right]-\left(\frac{k^{2}}{\varepsilon_{1} \varepsilon_{2}}\right)^{2} u^{2} \\
& +16 \varepsilon_{1}^{2} \varepsilon_{2}^{2}\left[(u-v)(1-u v) \xi_{0} n_{0}+2 v n_{0} \sin ^{2} \frac{1 \frac{1}{2} \phi}{}+\frac{k\left(\varepsilon_{1}+\varepsilon_{2}\right) u}{4 \varepsilon_{1}{ }^{2} \varepsilon_{2}{ }^{2}}\right]^{2}
\end{aligned}
$$


$B=16 \varepsilon_{1}{ }^{2} \varepsilon_{2}{ }^{2} v n_{0} \sin \phi\left[(u-v)(7-u v) \xi_{0} n_{0}+2 v n_{0} \sin ^{2{ }^{\frac{1}{2}} \phi}+\frac{k\left(\varepsilon_{1}+\varepsilon_{2}\right) u}{4 \varepsilon_{1}{ }^{2} \varepsilon_{2}{ }^{2}}\right]$

and

$$
16 \varepsilon_{1}{ }^{2} \varepsilon_{2}{ }^{2} v^{2} n_{0}{ }^{2} \sin ^{2} \phi \cdot A-B^{2}=16 \varepsilon_{1}{ }^{2} \varepsilon_{2}{ }^{2} v^{2} n_{0}{ }^{2} \sin ^{2} \phi\left[\begin{array}{l}
4 k^{2} \varepsilon_{1} \varepsilon_{2} \xi_{0} n_{0}\left[(u-v)^{2}+4 u v \sin ^{2} \frac{1}{2} \phi\right] \\
-\left(\frac{k^{2}}{\varepsilon_{1} \varepsilon_{2}}\right)^{2} u^{2}
\end{array}\right]
$$

Therefore

$$
\begin{aligned}
A C-B^{2} & =4 k^{2} \varepsilon_{1} \varepsilon_{2} \xi_{0} n_{0}\left[(u-v)^{2}+4 u v \sin ^{2}{ }_{\frac{1}{2} \phi}\right] \cdot A \\
& +16 \varepsilon_{1}^{2} \varepsilon_{2}^{2} v^{2} n_{0}^{2} \sin ^{2} \phi\left(4 k^{2} \varepsilon_{1} \varepsilon_{2} \xi_{0} n_{0}\left[(u-v)^{2}+4 u v \sin ^{2} \frac{1}{2} \phi\right]-\left(\frac{k^{2}}{\varepsilon_{1} \varepsilon_{2}}\right)^{2} u^{2}\right) \\
& =4 k^{2} \varepsilon_{1} \varepsilon_{2} \xi_{0} n_{0}\left[(u-v)^{2}+4 u v \sin ^{2}{ }^{2} \phi\right]
\end{aligned}
$$

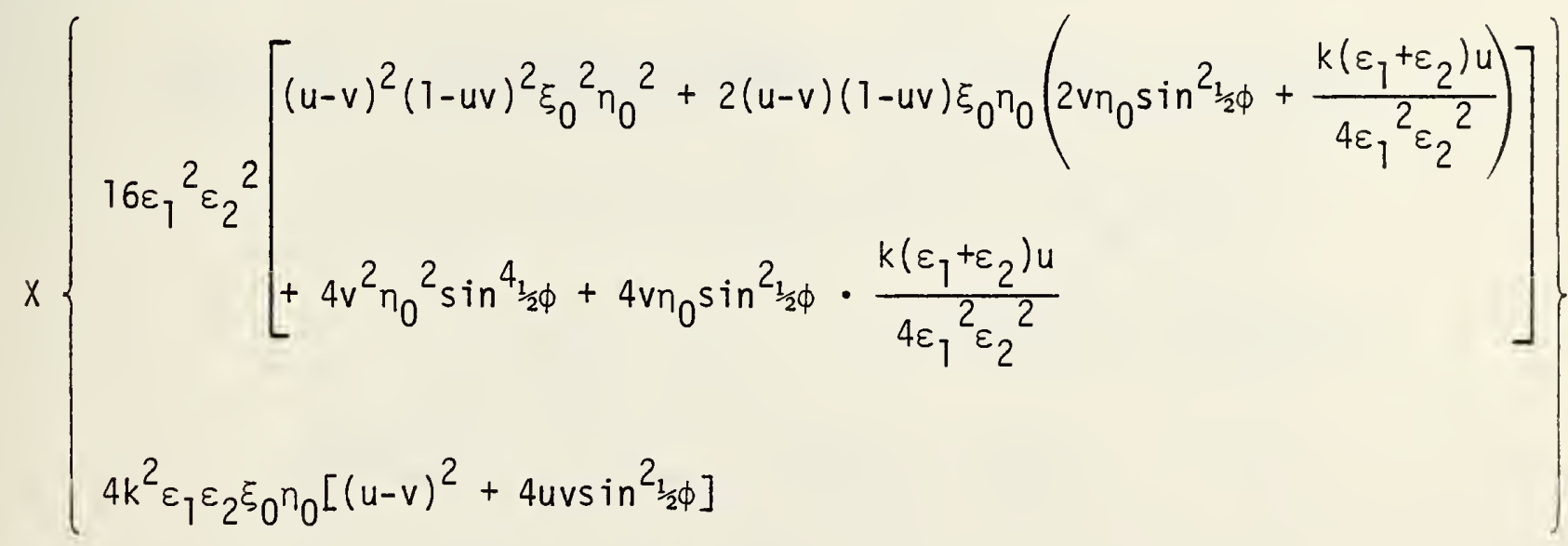$$
+4 k^{2} \varepsilon_{1} \varepsilon_{2} \xi_{0} n_{0}\left[(u-v)^{2}+4 u v \sin ^{2}{ }^{2} \frac{1}{2} \phi\right]\left[\frac{k^{2}\left(\varepsilon_{1}+\varepsilon_{2}\right)^{2} u^{2}}{\varepsilon_{1}^{2} \varepsilon_{2}^{2}}-\left(\frac{k^{2}}{\varepsilon_{1} \varepsilon_{2}}\right)^{2} u^{2}\right]
$$$$
+16 k^{2} \varepsilon_{1}^{2} \varepsilon_{2}^{2} v^{2} n_{0}{ }^{2} \sin ^{2} \phi \cdot 4 k^{2} \varepsilon_{7} \varepsilon_{2} \xi_{0} n_{0}\left[(u-v)^{2}+4 u v \sin ^{2} \frac{1}{2} \phi\right]
$$$$
-16 \varepsilon_{1}^{2} \varepsilon_{2}^{2} v^{2} n_{0}{ }^{2} \sin ^{2} \phi \cdot\left(\frac{k^{2}}{\varepsilon_{1} \varepsilon_{2}}\right)^{2} u^{2} \text {. }
$$ 
In the expression just given for $A C-B^{2}$, the term $4 v n_{0} \sin ^{2}{ }_{\frac{1}{2} \phi} \cdot \frac{k\left(\varepsilon_{1}+\varepsilon_{2}\right) u}{4 \varepsilon_{1}{ }^{2} \varepsilon_{2}{ }^{2}}$ in the large square bracket within the curly brackets is of order $1 / \varepsilon^{2}$ relative to other terms with a factor $\sin ^{2} \frac{1}{2} \phi$ and thus may be neglected. Next we note that the terms in the second square brackets in the second line may be simplified in that

$$
\frac{k^{2}\left(\varepsilon_{1}+\varepsilon_{2}\right)^{2} u^{2}}{\varepsilon_{1}{ }^{2} \varepsilon_{2}{ }^{2}}-\left(\frac{k^{2}}{\varepsilon_{1} \varepsilon_{2}}\right)^{2} u^{2}=\frac{4 k^{2} u^{2}}{\varepsilon_{1} \varepsilon_{2}} .
$$

The sum of the second and fourth 1 ines in eq (V.28) is therefore

$$
4 k^{2} \varepsilon_{1} \varepsilon_{2} \xi_{0} n_{0}(u-v)^{2} \frac{4 k^{2} u^{2}}{\varepsilon_{1} \varepsilon_{2}}+16 k^{4} u^{2}\left[\xi_{0} n_{0} 4 u v \sin ^{2}{ }_{\frac{1}{2} \phi}-v^{2} n_{0}{ }^{2} \sin ^{2} \phi\right] .
$$

The second line here is

$$
\begin{aligned}
& 16 k^{4} u^{2} \cdot 4 \sin ^{2 \frac{1}{2} \phi} \cdot v n_{0}\left[u \xi_{0}-v n_{0} \cos ^{2} \frac{1}{2} \phi\right] \\
& =16 k^{4} u^{2} \cdot 4 \sin ^{2}{ }_{\frac{1}{2}} \phi \cdot v n_{0}\left[\left(u \xi_{0}-v n_{0}\right)+v n_{0} \sin ^{2}{ }^{2} \frac{1}{2} \phi\right]
\end{aligned}
$$

Here $u \xi_{0}-v n_{0}=0\left((u-v) \xi_{0}\right)$. Therefore we have terms here of $O\left(\varepsilon^{4} \phi^{2}(u-v) / u\right)$ and $O\left(\varepsilon^{4} \phi^{4}\right)$. But we have other terms in $A C-B^{2}$, of $O\left(\varepsilon^{8} \xi_{0}{ }^{2} \phi^{4}\right)$. Therefore the terms of $O\left(\varepsilon^{4} \phi^{4}\right)$ may be dropped. We also have terms in $A C-B^{2}$ of order $\varepsilon^{6} \phi^{2} \xi_{0}(u-v) / u$. Therefore the terms 
of $0\left(\varepsilon^{4} \phi^{2}(u-v) / u\right)$ may be dropped. Thus, neglecting terms of relative orders $1 / \varepsilon^{2}$ and $\theta^{2}$, the only term to be retained from the second and fourth lines in eq (V.28) is

$$
4 k^{2} \varepsilon_{1} \varepsilon_{2} \xi_{0} n_{0}(u-v)^{2} \frac{4 k^{2} u^{2}}{\varepsilon_{1} \varepsilon_{2}} .
$$

We may therefore now write

$\frac{A C-B^{2}}{16 k^{2} \varepsilon_{1}{ }^{2} \varepsilon_{2}{ }^{2} \xi_{0}{ }^{2} n_{0}^{2}}=\left[(u-v)^{2}+4 u v \sin ^{2} \frac{1}{2} \phi\right]$

$$
\begin{aligned}
& x\left\{\begin{array}{c}
4 \varepsilon_{1} \varepsilon_{2}\left[\begin{array}{l}
(u-v)^{2}(1-u v)^{2} \xi_{0} n_{0} \\
+2(u-v)(1-u v)\left(2 v n_{0} \sin ^{2} \frac{1}{2} \phi+\frac{k\left(\varepsilon_{1}+\varepsilon_{2}\right) u}{4 \varepsilon_{1}{ }^{2} \varepsilon_{2}{ }^{2}}\right) \\
+\frac{4 v^{2} n_{0}{ }^{2}}{\xi_{0} n_{0}} \sin ^{4} \frac{1}{2} \phi
\end{array}\right] \\
+k^{2}\left[(u-v)^{2}+4 u v \sin ^{2}{ }_{\frac{1}{2} \phi}\right]
\end{array}\right] \\
& +(u-v)^{2}\left[\frac{k^{2} u^{2}}{\varepsilon_{1}{ }^{2} \varepsilon_{2}{ }^{2}}\left(1+u^{2}\right)\left(1+v^{2}\right)\right] \\
& +\left(\frac{v^{2} n_{0}{ }^{2} \sin ^{2} \phi}{\xi_{0} n_{0}}\right) \cdot 4 \varepsilon_{1} \varepsilon_{2}\left[(u-v)^{2}+4 u v \sin ^{2} \frac{1}{2} \phi\right] .
\end{aligned}
$$


In eq (V.29) the terms without $\phi^{2}$ are

$$
\begin{aligned}
& {\left[k^{2}+4 \varepsilon_{1} \varepsilon_{2}(1-u v)^{2} \xi_{0} n_{0}\right](u-v)^{2}\left(u-v+\frac{(1-u v) k\left(\varepsilon_{1}+\varepsilon_{2}\right) u}{\varepsilon_{1} \varepsilon_{2}\left[k^{2}+4 \varepsilon_{1} \varepsilon_{2}(1-u v)^{2} \xi_{0} n_{0}\right]}\right)^{2}} \\
& \quad+(u-v)^{2}\left(\frac{k u}{\varepsilon_{1} \varepsilon_{2}}\right)^{2}\left\{\left(1+u^{2}\right)\left(1+v^{2}\right)-\frac{(1-u v)^{2}\left(\varepsilon_{1}+\varepsilon_{2}\right)^{2}}{\left[k^{2}+4 \varepsilon_{1} \varepsilon_{2}(1-u v)^{2} \xi_{0} n_{0}\right]}\right\} .
\end{aligned}
$$

Here in the curly brackets we have

$$
\begin{aligned}
& \frac{1}{\left[k^{2}+4 \varepsilon_{1} \varepsilon_{2}(1-u v)^{2} \xi_{0} n_{0}\right]}\left\{\begin{array}{l}
k^{2}\left(1+u^{2}\right)\left(1+v^{2}\right)-k^{2}(1-u v)^{2} \\
-4 \varepsilon_{1} \varepsilon_{2}(1-u v)^{2}+4 \varepsilon_{1} \varepsilon_{2}(1-u v)^{2}
\end{array}\right\} \\
& =\frac{k^{2}(u+v)^{2}}{k^{2}+4 \varepsilon_{1} \varepsilon_{2}(1-u v)^{2} \xi_{0} n_{0}} .
\end{aligned}
$$

Thus the terms without $\phi^{2}$ are

$$
\begin{aligned}
& {\left[k^{2}+4 \varepsilon_{1} \varepsilon_{2}(1-u v)^{2} \xi_{0} n_{0}\right](u-v)^{2}\left(u-v+\frac{(1-u v) k\left(\varepsilon_{1}+\varepsilon_{2}\right) u}{\varepsilon_{1} \varepsilon_{2}\left[k^{2}+4 \varepsilon_{1} \varepsilon_{2}(1-u v)^{2} \xi_{0} n_{0}\right]}\right)^{2}} \\
& +\left(\frac{k^{2} u}{\varepsilon_{1} \varepsilon_{2}}\right)^{2}(u-v)^{2} \frac{(u+v)^{2}}{\left[k^{2}+4 \varepsilon_{1} \varepsilon_{2}(1-u v)^{2} \xi_{0} n_{0}\right]}
\end{aligned}
$$


Next the terms in eq (v.29) with factors $(u-v)$ and $\sin ^{2} \frac{1}{2} \phi$ are $4 u v \sin ^{2} \frac{1}{2} \phi \cdot(u-v)^{2}\left[2 k^{2}+4 \varepsilon_{1} \varepsilon_{2}(1-u v)^{2} \xi_{0} n_{0}\right]$

$+4 \varepsilon_{1} \varepsilon_{2}(u-v)^{2}\left(\frac{v^{2} n_{0}{ }^{2} \sin ^{2} \phi}{\xi_{0} n_{0}}+\frac{4 v^{2} n_{0}{ }^{2} \sin ^{4 \frac{1}{2} \phi}}{\xi_{0} n_{0}}+2(u-v)(1-u v) \cdot 2 v n_{0} \sin ^{2} \frac{1 / 2}{2}\right)$

$+4 \varepsilon_{1} \varepsilon_{2} \cdot 4 u v \sin ^{2} \frac{1}{2} \phi \cdot 2(u-v)(1-u v)\left[\frac{k\left(\varepsilon_{1}+\varepsilon_{2}\right) u}{4 \varepsilon_{1}{ }^{2} \varepsilon_{2}{ }^{2}}+2 u v n_{0} \sin ^{2} \frac{1}{2} \phi\right]$

Here the second line may be written as

$$
\begin{aligned}
4 \varepsilon_{1} \varepsilon_{2}(u-v)^{2} & =4 v n_{0} \sin ^{2} \frac{1}{2} \phi\left(\frac{v n_{0}}{\xi_{0} n_{0}} \cos ^{2} \frac{1}{2} \phi+\frac{v n_{0}}{\xi_{0} n_{0}} \sin ^{2} \frac{1}{\frac{1}{2}} \phi+(u-v)(1-u v)\right) \\
& =16 \varepsilon_{1} \varepsilon_{2} v n_{0}(u-v)^{2} \sin ^{2 \frac{1}{2} \phi}\left(v\left(1+u^{2}\right)+(u-v)(1-u v)\right) \\
& =16 \varepsilon_{1} \varepsilon_{2} v n_{0}(u-v)^{2} \sin ^{2} \frac{1}{\frac{1}{2}} \phi\left(u\left(1+v^{2}\right)\right) \\
& =16 \varepsilon_{1} \varepsilon_{2}(u-v)^{2} u v \sin ^{2} \frac{1}{\frac{1}{2} \phi} .
\end{aligned}
$$

Thus the terms in eq (V.29) with factors $(u-v)$ and $\sin ^{2} \frac{1}{2} \phi$ are 


$$
\begin{aligned}
& \text { 4uvsin }{ }^{2} \frac{1}{2} \phi \cdot(u-v)^{2}\left[2 k^{2}+4 \varepsilon_{1} \varepsilon_{2}(1-u v)^{2} \xi_{0} n_{0}+4 \varepsilon_{1} \varepsilon_{2}\right] \\
& +4 u v \sin ^{2} \frac{1}{2} \phi \cdot 2(u-v)(1-u v) \cdot 4 \varepsilon_{1} \varepsilon_{2}\left[2 v n_{0} \sin ^{2} \frac{1}{\frac{1}{2} \phi}+\frac{k\left(\varepsilon_{1}+\varepsilon_{2}\right) u}{4 \varepsilon_{1}{ }^{2} \varepsilon_{2}{ }^{2}}\right] .
\end{aligned}
$$

Here we write

$$
\begin{aligned}
(1-u v)^{2} \xi_{0} n_{0}+1 & =2(1-u v)^{2} \xi_{0} n_{0}+1-(1-u v)^{2} \xi_{0} n_{0} \\
& =2(1-u v)^{2} \xi_{0} n_{0}+\xi_{0} n_{0}\left[\left(1+u^{2}\right)\left(1+v^{2}\right)-(1-u v)^{2}\right] \\
& =2(1-u v)^{2} \xi_{0} n_{0}+\xi_{0} n_{0}(u+v)^{2} .
\end{aligned}
$$

Thus we have, for the terms with factors $(u-v)$ and $\sin ^{2} \frac{1}{2} \phi$,

$$
\begin{aligned}
& 2 \cdot 4 u v \sin ^{2} \frac{1}{2} \phi \cdot(u-v)^{2}\left[2 k^{2}+4 \varepsilon_{1} \varepsilon_{2}(1-u v)^{2} \xi_{0} n_{0}\right] \\
& +4 u v \sin ^{2} \frac{1}{2} \phi \cdot(u-v)^{2} \cdot 4 \varepsilon_{1} \varepsilon_{2}(u+v)^{2} \xi_{0} n_{0} \\
& +2 \cdot 4 u v \sin ^{2} \frac{1}{2} \phi \cdot(u-v) \cdot(1-u v) \frac{k\left(\varepsilon_{1}+\varepsilon_{2}\right) u}{\varepsilon_{1} \varepsilon_{2}} \\
& +4 u v \sin ^{2} \frac{1}{2} \phi \cdot 2(u-v)(1-u v) \cdot 4 \varepsilon_{1} \varepsilon_{2} \cdot 2 v n_{0} \sin ^{2 \frac{1}{2} \phi}
\end{aligned}
$$


$=2(u-v)\left(u-v+(1-u v) \frac{k\left(\varepsilon_{1}+\varepsilon_{2}\right) u}{\varepsilon_{1} \varepsilon_{2}\left[k^{2}+4 \varepsilon_{1} \varepsilon_{2}(1-u v)^{2} \xi_{0} n_{0}\right]}\right) 4 u v \sin ^{2}{ }^{\frac{1}{2} \phi}\left[k^{2}+4 \varepsilon_{1} \varepsilon_{2}(1-u v)^{2} \xi_{0} n_{0}\right]$

$+4 u v \sin ^{2}{ }^{2} \frac{1}{2} \phi\left[(u-v)^{2} \cdot 4 \varepsilon_{1} \varepsilon_{2}(u+v)^{2} \xi_{0} n_{0}+2(u-v)(1-u v) \cdot 4 \varepsilon_{1} \varepsilon_{2} \cdot 2 v n_{0} \sin ^{2^{2}}{ }^{2} \phi\right]$.

Finally, we have the terms in eq (v.29) without a factor $(u-v)$ :

$$
\begin{aligned}
& \operatorname{uuvsin}^{2}{ }_{\frac{1}{2} \phi}\left[\begin{array}{l}
4 \varepsilon_{1} \varepsilon_{2} \cdot \frac{4 v^{2} n_{0}{ }^{2}}{\varepsilon_{0} \varepsilon_{0}} \sin ^{4 \frac{l_{2}}{2} \phi}+k^{2} \cdot 4 u v \sin ^{2}{ }_{\frac{1}{2} \phi} \\
+4 \varepsilon_{1} \varepsilon_{2} \cdot \frac{v^{2} n_{0}{ }^{2}}{\xi_{0} n_{0}} \sin ^{2} \phi
\end{array}\right] \\
& =4 u v \sin ^{2}{ }_{\frac{1}{2} \phi}\left[4 \varepsilon_{1} \varepsilon_{2} \cdot \frac{4 v^{2} n_{0}^{2}}{\xi_{0} n_{0}} \sin ^{2}{ }_{\frac{1}{2} \phi}+k^{2} \cdot 4 u v \sin ^{2}{ }_{\frac{1}{2} \phi}\right] \\
& =16 u v \sin ^{4}{ }_{\frac{1}{2} \phi}\left[4 \varepsilon_{1} \varepsilon_{2} \frac{v^{2} n_{0}}{\xi_{0}}+k^{2} u v\right] .
\end{aligned}
$$

We must now combine the terms given in (V.30), (V.31), and (V.32). For this purpose it is convenient to designate by (V.30a) and (V.30b) the first and second terms in (V.30), and, in similar fashion, to designate by (V.37a) and (V.310) the first and second terms in (V.31). We then have 
$(v .30 a)+(v .37 a)$

$$
\begin{aligned}
& =\left[k^{2}+4 \varepsilon_{7} \varepsilon_{2}(1-u v)^{2} \xi_{0} n_{0}\right]\left\{\begin{array}{l}
\left.(u-v)\left(u-v+\frac{(1-u v) k\left(\varepsilon_{7}+\varepsilon_{2}\right) u}{\varepsilon_{7} \varepsilon_{2}\left[k^{2}+4 \varepsilon_{7} \varepsilon_{2}(1-u v)^{2} \xi_{0} n_{0}\right]}\right)\right\}^{2} \\
+4 u v \sin ^{2} \frac{1}{2} \phi
\end{array}\right. \\
& -\left[k^{2}+4 \varepsilon_{1} \varepsilon_{2}(1-u v)^{2} \xi_{0} n_{0}\right]\left(4 u v \sin ^{2} \frac{1}{2} \phi\right)^{2}
\end{aligned}
$$

Adding ( $V .32)$ to the second term in ( $V .33$ ) gives

$$
\begin{aligned}
\text { T6uvsin }{ }^{4 \frac{1}{2} \phi}\left[4 \varepsilon_{1} \varepsilon_{2} \frac{v^{2} n_{0}}{\xi_{0}}-4 \varepsilon_{1} \varepsilon_{2}(1-u v)^{2} \xi_{0} n_{0} u v\right] \\
=64 \varepsilon_{1} \varepsilon_{2} u v \sin ^{4} \frac{1 / \frac{1}{2} \phi}{v_{0}}\left[\frac{v^{2} n_{0}}{\xi_{0}}-(1-u v)^{2} \xi_{0} n_{0} u v\right],
\end{aligned}
$$

and adding this to $(V .31 \mathrm{~b})$ gives

$$
\begin{aligned}
& 64 \varepsilon_{1} \varepsilon_{2} u v \sin ^{4}{ }_{\frac{1}{2} \phi}\left[\frac{v^{2} n_{0}}{\xi_{0}}-(1-u v)^{2} \xi_{0} n_{0} u v+(u-v)(1-u v) v n_{0}\right] \\
& +4 u v \sin ^{2} \frac{1}{2} \phi(u-v)^{2} \cdot 4 \varepsilon_{1} \varepsilon_{2}(u+v)^{2} \xi_{0} n_{0}
\end{aligned}
$$

The expression in the square brackets in (V.34) may be simplified: We have there 


$$
\begin{aligned}
& v n_{0}\left[\frac{v}{\xi_{0}}-(1-u v)^{2} u \xi_{0}+(u-v)(1-u v)\right] \\
& \quad=v \xi_{0} n_{0}\left[v\left(1+u^{2}\right)^{2}-(1-u v)^{2} u+(u-v)(1-u v)\left(1+u^{2}\right)\right] \\
& =v \xi_{0} n_{0}\left[v+2 v u^{2}+v u^{4}-u+2 u^{2} v-u^{3} v^{2}+u-u^{2} v+u^{3}-u^{4} v-v+u v^{2}-v u^{2}+v^{2} u^{3}\right] \\
& =u v \xi_{0} n_{0}\left[u^{2}+2 u v+v^{2}\right] \\
& =u v \xi_{0} n_{0}(u+v)^{2}
\end{aligned}
$$

Thus the expression (V.34) may be written in the form

$$
4 \varepsilon_{1} \varepsilon_{2}\left(4 u v \sin ^{2} \frac{1}{2} \phi\right)^{2} \xi_{0} n_{0}(u+v)^{2}+4 \varepsilon_{1} \varepsilon_{2}\left(4 u v \sin ^{2} \frac{1}{2} \phi\right) \xi_{0} n_{0}(u-v)^{2}(u+v)^{2} .
$$

Adding this to ( $V .30 \mathrm{~b})$ then gives

$$
4 \varepsilon_{1} \varepsilon_{2} \xi_{0} n_{0}(u+v)^{2}\left\{\begin{array}{l}
\left(4 u v \sin ^{2} \frac{1}{2} \phi\right)\left[(u-v)^{2}+4 u v \sin ^{2}{ }_{\frac{1}{2} \phi}\right] \\
+\left(\frac{k^{2} u}{\varepsilon_{1} \varepsilon_{2}}\right)^{2} \frac{(u-v)^{2}}{4 \varepsilon_{1} \varepsilon_{2} \xi_{0} n_{0}\left[k^{2}+4 \varepsilon_{7} \varepsilon_{2}(1-u v)^{2} \xi_{0} n_{0}\right]}
\end{array}\right\} .
$$

Finally, combining (V.35) with the first term in (V.33) gives our desired form for $A C-B^{2}$, given previously by (V.29), viz., 


$$
A C-B^{2}=16 k^{2} \varepsilon_{1}^{2} \varepsilon_{2}^{2} \xi_{0}^{2} n_{0}^{2}
$$

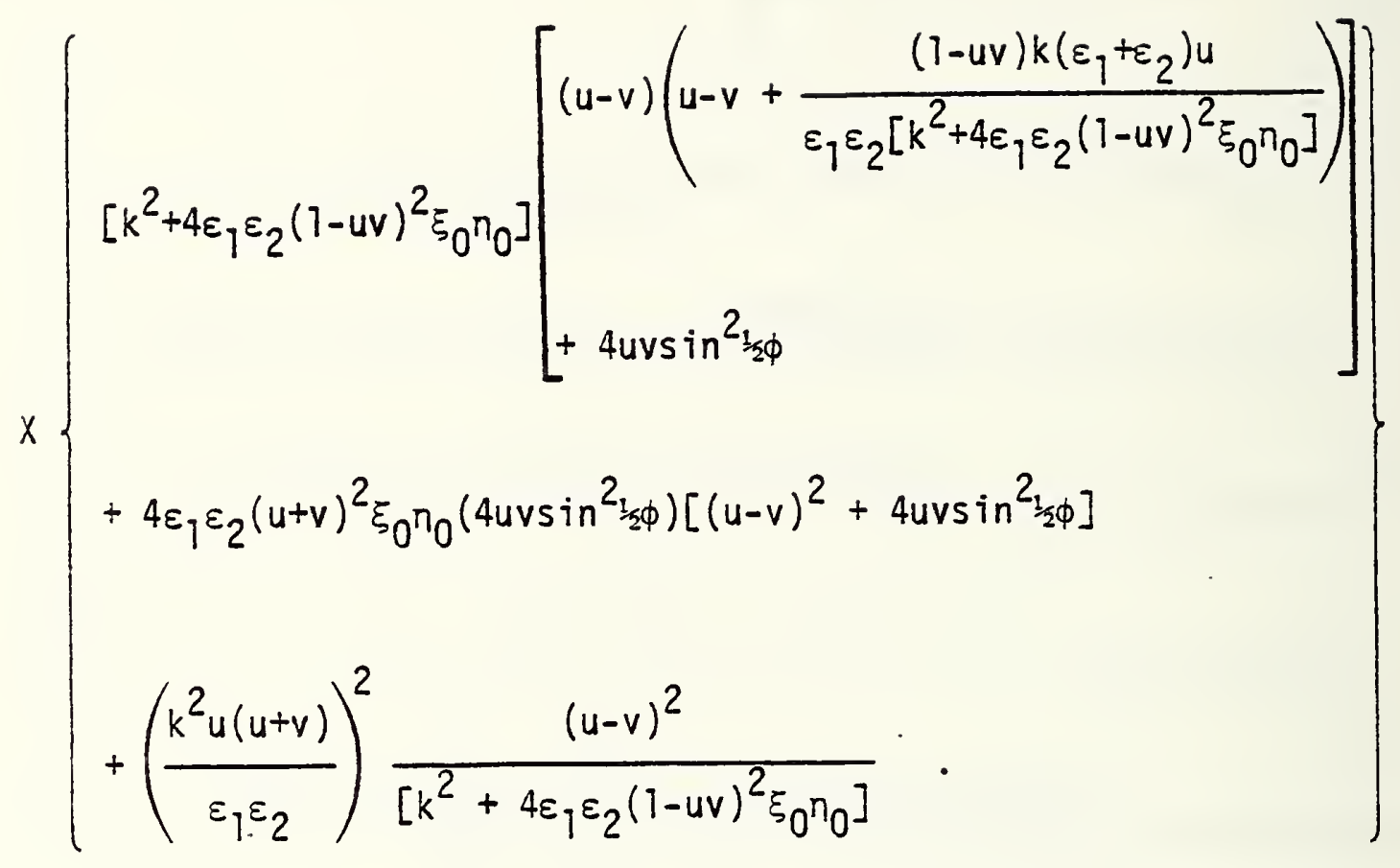

This shows that $A C-B^{2} \geqq 0$, which, along with $A \geqq 0$ and $C \geqq 0$, completes the task of writing the high energy small angle expression for in a form that is manifestly positive.

We now write our high energy small angle approximation for the numerators \{\}$_{1}$ and \{\}$_{\|}$appearing in the cross sections $d \sigma_{\perp}$ and $d \sigma_{1}$ (eggs (I.2), (I.3), (IV.9), and (IV.10)), in a form similar to that given for the numerator of the cross section for unpolarized photons in eq (VI.49) on p. 66 of [1]. There we wrote

$$
\{\}=f(v)(v-u+\sigma)^{2}+g(v) \sin ^{2} \frac{1}{2} \phi+\rho
$$

From eqs (IV.9) and (V.22) we have

$$
\{\}_{1}=f_{1}(v)(v-u)^{2}+g_{1}(v) \sin ^{2} i_{\frac{1}{2}} \phi
$$

44 
where

$$
f_{1}(v)=\frac{4 k^{2} \varepsilon_{1} \varepsilon_{2}}{\left(1+u^{2}\right)\left(1+v^{2}\right)}
$$

and

$$
g_{1}(v)=\frac{16 k^{2} \varepsilon_{1} \varepsilon_{2} u v}{\left(1+u^{2}\right)\left(1+v^{2}\right)}+\frac{64 \varepsilon_{1}{ }^{2} \varepsilon_{2}{ }^{2} v^{2} \cos ^{2} \frac{1}{2} \phi}{\left(1+v^{2}\right)^{2}},
$$

and from eqs (IV.10) and (V.25) we have

$$
\{\}_{\|}=f_{\|}(v)\left(v-u+\sigma_{\|}\right)^{2}+g_{\|}(v) \sin ^{2} \frac{1}{2} \phi+\rho_{\|}
$$

where

$$
\begin{gathered}
f_{\|}(v)=\frac{4 \varepsilon_{1}^{2} \varepsilon_{2}^{2}}{\left(1+u^{2}\right)\left(1+v^{2}\right)}\left[\frac{k^{2}}{\varepsilon_{1} \varepsilon_{2}}+4 \frac{(u v-1)^{2}}{\left(1+u^{2}\right)\left(1+v^{2}\right)}\right] \\
g_{\|}(v)=\frac{16 k^{2} \varepsilon_{1} \varepsilon_{2} u v}{\left(1+u^{2}\right)\left(1+v^{2}\right)}+\frac{64 k^{2} \varepsilon_{1} \varepsilon_{2} v^{2} \sin ^{2} \frac{1}{2} \phi}{\left(1+v^{2}\right)^{2}\left[\frac{k^{2}}{\varepsilon_{1} \varepsilon_{2}}+4 \frac{(u v-1)^{2}}{\left(1+u^{2}\right)\left(1+v^{2}\right)}\right]}
\end{gathered}
$$




$$
\sigma_{\|}=\sigma_{\|}(v)=\frac{4(u v-1)\left[2 v n_{0} \sin ^{2 \frac{1}{2} \phi}+\frac{k\left(\varepsilon_{7}+\varepsilon_{2}\right) u}{4 \varepsilon_{7}^{2} \varepsilon_{2}^{2}}\right]}{\left[\frac{k^{2}}{\varepsilon_{1} \varepsilon_{2}}+4 \frac{(u v-1)^{2}}{\left(1+u^{2}\right)\left(1+v^{2}\right)}\right]}
$$

and

$$
\rho_{\| 1}=\frac{16\left(\frac{k^{2}}{\varepsilon_{1} \varepsilon_{2}}\right)^{2} u^{4}}{\left(1+u^{2}\right)^{2}\left[\frac{k^{2}}{\varepsilon_{1} \varepsilon_{2}}+4\left(\frac{u^{2}-1}{u^{2}+1}\right)^{2}\right]} .
$$

In comparing eq (V.44) with eq (V.25) it will be noticed that in eq (v.44) we have set $v=u$ throughout. Since $\rho_{\| l}$ is of $0(1)$, it is negligible unless both $u-v=0\left(u \theta^{2}\right)$ and $\phi=0\left(\theta^{2}\right)$. We may therefore set $v=u$ in $\rho_{\| l}$ as it appears in eq ( $\left.v .25\right)$, with neglect of terms of relative order $\theta^{2}$.

The expressions (V.37) and (V.40) for the numerators \{\}$_{1}$ and \{\}$_{\|}$will be of particular use in the following section, where we determine the maxima and minimum of the cross sections $d \sigma_{\perp}$ and $d \sigma_{\|}$ in the region of sma 11 momentum transfers, $q \lesssim 0(u \theta)$ (i.e., $q=0(u \theta)$ and $\left.q=0\left(u \theta^{2}\right)\right)$. 
VI. DETERMINATION OF THE MAXIMA AND MINIMUM OF THE POLARIZED BREMSSTRAHLUNG CROSS SECTIONS $\mathrm{d}_{1}$ AND $\mathrm{do} \|$

In this section we consider the cross sections $d \sigma_{1}$ and $d \sigma_{\|}$as functions of $v$ (i.e., $\theta_{2}$ ) for fixed values of the other variables $\left(\varepsilon_{1}, \varepsilon_{2}, \theta_{1}\right.$ and $\left.\phi\right)$ and determine the value of $v$ for which these cross sections achieve their maximum and minimum values. In order to locate the extrema of the cross sections and evaluate the cross sections at these extrema we use the high energy small angle approximations for \{\}$_{1}$ and \{\}$_{\|}$given in eqs (V.37) and (V.40), and for $q^{2}$, given in eq $(V .15)$ of $[1]$, viz.,

$$
q^{2}=(v-u)^{2}+4 u v \sin ^{2} \frac{1}{2} \phi+\frac{2 \delta\left(1+u^{2}\right) u(v-u)}{\varepsilon_{2}}+\delta^{2}\left(1+u^{2}\right)^{2}
$$

where $\delta=k /\left(2 \varepsilon_{1} \varepsilon_{p}\right)$. We will follow very closely the analysis presented in detail in section VII of [1], and will therefore make frequent reference to equations and discussion given there. As discussed there on pp. 72 and 73 , we also neglect screening in this analysis in determining the location of the extrema of the cross sections, setting $F(q)=0$ in eqs (I.2) and (I.3). The determining equations for the extrema are then (see eq (VII.2a), p. 73 of [1])

$$
q^{2}\{\}_{1}^{\prime}=2\left(q^{2}\right)^{\prime}\{\}_{1}
$$

and

$$
q^{2}\{\}_{\|}^{\prime}=2\left(q^{2}\right)^{\prime}\{\}_{\|} .
$$


As in [1], we are looking for maxima and minima in the region of small $q$, i.e., $q \lesssim 0(u \theta)$, and hence also $q_{1} \lesssim 0(u \theta)$. From $q_{1}^{2}=(\underline{u}-\underline{v})^{2}=$ $(u-v)^{2}+4 u v \sin ^{2} \frac{1}{2} \phi$ we have $|u-v| \leqq q_{1}$ and $u \phi \leqq 0\left(q_{1}\right)$. Thus for $q_{1} \lesssim O(u \theta)$ we have $\frac{v-u}{u} \lesssim 0(\theta)$ and $\phi \lesssim 0(\theta)$. We therefore assume in this section, where $q \lesssim 0(u \theta)$, that $\phi \lesssim 0(\theta)$, neglecting terms of relative order $\phi^{2} \lesssim O(\theta)$ in our high energy small angle approximations for $q^{2},\{\}_{1},\{\}_{\|}$, and their derivatives with respect to $v$. This results in considerable simplification in the expressions for \{\}$_{\perp}$ and \{\}$_{\|}($eqs $(V .37)$ and $(V .40)$ ), i.e., in the eqs (V.38) and (V.39) for $f_{\perp}(v)$ and $g_{1}(v)$, and in eqs $(v .41)-(V .44)$ for $f_{\| l}(v)$, $g_{\|}(v), \quad \sigma_{\|}(v)$, and $\rho_{\|}$. Thus, neglecting terms of relative order $\phi^{2}$ in eqs (V.39) and (V.42), we now have, for $q \lesssim 0(u \theta)$,

$$
g_{1}(v)=\frac{16 k^{2} \varepsilon_{1} \varepsilon_{2} u v}{\left(1+u^{2}\right)\left(1+v^{2}\right)}+\frac{64 \varepsilon_{1}{ }^{2} \varepsilon_{2}{ }^{2} v^{2}}{\left(1+v^{2}\right)^{2}}
$$

and

$$
g_{\|}(v)=\frac{16 k^{2} \varepsilon_{1} \varepsilon_{2} u v}{\left(1+u^{2}\right)\left(1+v^{2}\right)} .
$$

Referring to eq (v.43), we see that the function $\sigma_{\|}(v)$ is of $0\left(u \theta^{2}\right)$ for $\phi \lesssim 0(\theta)$. Moreover, $\sigma_{\|}(v)$ appears in the term $\left(v-u+\sigma_{\|}\right)^{2}$ in eq $(v .40)$, where now $v-u \lesssim 0(u \theta)$. Now if, for $v-u=0(u \theta)$, we set $v=u$ in the expression (v.43) for $\sigma_{\|}(v)$, then we introduce errors of $0((v-u) / u)=0(\theta)$ relative to $\sigma_{\|}(v)$. These errors are thus of $O\left(u \theta^{3}\right)$; i.e., they are of $0\left(\theta^{2}\right)$ relative to $v-u$, and hence may be neglected. On the other hand, for $v-u=0\left(u \theta^{2}\right)$, setting $v=u$ in 
eq (V.43) introduces errors of order $\theta^{2}$ relative to $\sigma_{\|}(v)$. Once again these may be neglected. Therefore, for $q \lesssim 0(u \theta)$ we may, in eq (v.43), set $v=u$ and neglect terms of relative order $\phi^{2}$. We then obtain

$$
\sigma_{\| 1}=\frac{u\left(u^{2}-1\right)\left[\frac{2 \phi^{2}}{1+u^{2}}+\frac{k\left(\varepsilon_{1}+\varepsilon_{2}\right)}{\varepsilon_{1}{ }^{2} \varepsilon_{2}{ }^{2}}\right]}{\left[\frac{k^{2}}{\varepsilon_{1} \varepsilon_{2}}+4\left(\frac{u^{2}-1}{u^{2}+1}\right)^{2}\right]} \text {. }
$$

The expression (VI.6), valid in the region $q \lesssim O(u \theta)$, now closely resembles the expression for $\sigma$ given in eq (VI.48) on p. 66 of [1]. The two expressions are independent of $v$, and are of the same order of magnitude, viz., $O\left(u \theta^{2}\right)$.

Thus for $q \lesssim O(u \theta), f_{\perp}(v)$ and $f_{\|}(v)$ as given in eqs (v.38) and (V.41) are each of the same form as $f(v)$, given in eq (VI.44) on p. 62 of [1], all three being of $0\left(\varepsilon^{4} \xi^{2}\right)$, and $g_{1}(v)$ and $g_{\|}(v)$ as given in eqs (VI.4) and (VI.5) are each of the same form as $g(v)$, given in eq (VI.45) on p. 62 of [1], all three being of $0\left(\varepsilon^{4} \xi^{2} u^{2}\right)$. Further, as just noted, again provided that $q \leqq 0(u \theta), \sigma_{\|}$as given in eq (VI.6) is of the same order of magnitude as $\sigma$, given in eq (V.48) on p. 66 of [1], and $\rho_{\|}$as given in eq (V.44) is of the same form and order of magnitude as $\rho$, given in eq (VI.47) on p. 65 of [1]. (The fact that for large $u \gg 1, \rho$ is larger than $\rho_{\|}$by a factor $u^{2}$ is of no significance for the analysis. In both cases, they need be retained oniy when both $v-u=o\left(u \theta^{2}\right)$ and $\phi=O\left(\theta^{2}\right)$.)

It thus follows that if we define 


$$
\sigma_{1} \equiv 0
$$

and

$$
\rho_{1} \equiv 0
$$

then we can, for $q \leqslant 0(u \theta)$, write \{\}$_{\perp}$ and \{\}$_{\|}$in the form given previously for \{\} in eq (VI.49) on p. 66 of [1]:

$$
\{\}_{\perp, \|}=f_{\perp, \|}(v)\left(v-u+\sigma_{1, \|}\right)^{2}+g_{1, \|}(v) \sin ^{2} \frac{1}{2} \phi+\rho_{\perp, \|} .
$$

And, since the form and the order of magnitude of the quantities appearing here are the same as those in eq (VI.49) of [1], we can utilize without modification most of the analysis presented in sec. VII of [1]. There we defined ((VII.3) in [l])

$$
z=\frac{v-u}{u}
$$

and looked for solutions of the equation defining the extrema ((VII.2a) in [I], eqs (VI.2) and (VI.3) here), first in the region $z \lesssim 0\left(\theta^{2}\right)$, and then in the region $z=0(\theta)$. We found that for sufficientiy small $\phi$, the equation defining the extrema, considered as a function of $z$ (or $v$ ), had one solution in the region $z \lesssim 0\left(\theta^{2}\right)$, corresponding to the minimum of the cross section, and two solutions in the region $z=0(\theta)$, corresponding to the maxima of the cross section, one for $z<0 \quad(v<u)$ and one for $z>0 \quad(v>u)$. Further, we found that as $\phi$ increases, the position of the minimum moves to larger values of $v$, 
and the position of the second maximum (the one for $z>0$ ) moves to smaller values of $v$. When $\phi$ reaches a critical value, $\phi_{C}$, the minimum and the second maximum occur at the same point, which is then an inflection point of the cross section. This point occurs for $z=0\left(\theta^{4 / 3}\right)$. For $\phi>\phi_{c}$ there is only one extremum, the maximum which occurs for $v<u$. This behavior of the extrema as a function of $\phi$ was shown in figures 11 and 12 in [1]. For polarized photons, identical statements may be made for $d \sigma_{\perp}$. However, the behavior of $d \sigma_{\|}$depends on the photon energy, $k$. For $k$ satisfying (VI.25) it is the same as that of $d \sigma_{1}$, whereas for $k$ satisfying (VI.33) $d \sigma_{\|}$has a sharp dip for all values of $\phi$. (See figs. $4(a)-4(d)$ and $5(\mathrm{a})-5(\mathrm{~d})$. )

Thus for $z \leqq 0\left(\theta^{2}\right)$ and $\phi$ arbitrary (but still assuming $\phi \leqq 0(\theta)$ ), we have, directly from eq (VII.7) on p. 78 of [1], the position of the minima for the cross sections $d \sigma_{\perp}$ and $d \sigma_{\|}, z_{\text {minl }}$ and $z_{\text {minll }}$, respectively:

$z_{\min 1}=-\frac{\left(\frac{\sigma_{1} f_{1}(u)}{u}+\frac{g_{\perp}{ }^{\prime}(u)}{u} \frac{\phi^{2}}{8}\right)\left(\phi^{2}+\frac{\delta^{2}\left(1+u^{2}\right)^{2}}{u^{2}}\right)-\frac{g_{1}(u)}{u^{2}} \frac{\phi^{2}}{2}\left(\frac{\phi^{2}}{2}+\frac{\delta\left(1+u^{2}\right)}{\varepsilon_{2}}\right)}{f_{\perp}(u)\left(\phi^{2}+\frac{\delta^{2}\left(1+u^{2}\right)^{2}}{u^{2}}\right)-\frac{g_{1}(u)}{u^{2}} \frac{\phi^{2}}{2}}$

and

$$
z_{\text {minin }}=-\frac{\left(\frac{\sigma_{\|} f_{\|}(u)}{u}+\frac{g_{\|}{ }^{\prime}(u)}{u} \frac{\phi^{2}}{8}\right)\left(\phi^{2}+\frac{\delta^{2}\left(1+u^{2}\right)^{2}}{u^{2}}\right)-\frac{g_{\|}(u)}{u^{2}} \frac{\phi^{2}}{2}\left(\frac{\phi^{2}}{2}+\frac{\delta\left(1+u^{2}\right)}{\varepsilon_{2}}\right)}{f_{\|}(u)\left(\phi^{2}+\frac{\delta^{2}\left(1+u^{2}\right)^{2}}{u^{2}}\right)-\frac{g_{\|}(u)}{u^{2}} \frac{\phi^{2}}{2}}
$$


Noting from eq (VI.7) that we defined $\sigma_{1}=0$, the expression (VI.11) for $z_{\text {minl }}$ then'simplifies somewhat and may be written in the form

$$
z_{\operatorname{minL}}=-\phi^{2} \frac{\left[\frac{g_{1}{ }^{\prime}(u)}{8 u f_{1}(u)}\left(\phi^{2}+\frac{\delta^{2}\left(1+u^{2}\right)^{2}}{u^{2}}\right)-\frac{g_{1}(u)}{2 u^{2} f_{\perp}(u)}\left(\frac{\phi^{2}}{2}+\frac{\delta\left(1+u^{2}\right)}{\varepsilon_{2}}\right)\right]}{\left[\frac{\delta^{2}\left(1+u^{2}\right)^{2}}{u^{2}}-\left(\frac{g_{1}(u)}{2 u^{2} f_{\perp}(u)}-1\right) \phi^{2}\right]} .
$$

The expression (VI.12) for $z_{\text {minll }}$ may be written in the closely similar form

$$
z_{\text {min } \|}=-\frac{\left(\frac{g_{\|}{ }^{\prime}(u) \phi^{2}}{8 u f_{\|}(u)}+\frac{\sigma_{\|}}{u}\right)\left(\phi^{2}+\frac{\delta^{2}\left(1+u^{2}\right)^{2}}{u^{2}}\right)-\frac{g_{\|}(u) \phi^{2}}{2 u^{2} f_{\|}(u)}\left(\frac{\phi^{2}}{2}+\frac{\delta\left(1+u^{2}\right)}{\varepsilon_{2}}\right)}{\left[\frac{\delta^{2}\left(1+u^{2}\right)^{2}}{u^{2}}-\left(\frac{g_{\|}(u)}{2 u^{2} f_{\|}(u)}-1\right) \phi^{2}\right]} .
$$

We consider first the expression (VI.13) for $z_{\text {minl }}$. Noting from eqs (VI.4) and (V.38) that

$$
\frac{g_{1}(u)}{2 u^{2} f_{\perp}(u)}=\frac{2 k^{2}+8 \varepsilon_{1} \varepsilon_{2}}{k^{2}} \text {, }
$$


we define, in analogy with eq (VII.10) on p. 80 of [1],

$$
\begin{aligned}
\phi_{01} & =\frac{\delta\left(1+u^{2}\right)}{u}\left(\frac{g_{1}(u)}{2 u^{2} f_{1}(u)}-1\right)^{-\frac{1}{2}} \\
& =\frac{\delta\left(1+u^{2}\right)}{u}\left(\frac{k^{2}}{k^{2}+8 \varepsilon_{1} \varepsilon_{2}}\right)^{\frac{1}{2}} .
\end{aligned}
$$

Further, from eq (VI.4) we have

$$
g_{1}^{\prime}(v)=\frac{16 k^{2} \varepsilon_{1} \varepsilon_{2} u}{\left(1+u^{2}\right)}\left(\frac{1-v^{2}}{\left(1+v^{2}\right)^{2}}\right)+128 \varepsilon_{1}{ }^{2} \varepsilon_{2}^{2}\left(\frac{v}{1+v^{2}}\right)\left(\frac{1-v^{2}}{\left(1+v^{2}\right)^{2}}\right)
$$

and thus, with $f_{1}(u)$ as given by eq (v.38),

$$
\frac{g_{1}^{\prime}(u)}{u f_{1}(u)}=4\left(\frac{1-u^{2}}{1+u^{2}}\right)\left(\frac{k^{2}+8 \varepsilon_{1} \varepsilon_{2}}{k^{2}}\right) \text {. }
$$

Substituting eqs (VI.15), (VI.16), and (VI.17) in (VI.13), we may write the expression for $z_{\text {minl }}$ in the form

$$
z_{\text {minL }}=\phi^{2} \frac{\left[\frac{1}{2}\left(\frac{u^{2}-1}{u^{2}+1}\right)\left(\phi^{2}+\frac{\delta^{2}\left(1+u^{2}\right)^{2}}{u^{2}}\right)+2\left(\frac{k^{2}+4 \varepsilon_{1} \varepsilon_{2}}{k^{2}+8 \varepsilon_{1} \varepsilon_{2}}\right)\left(\frac{\phi^{2}}{2}+\frac{\delta\left(1+u^{2}\right)}{\varepsilon_{2}}\right)\right]}{\left(\phi_{0 \perp}^{2}-\phi^{2}\right)}
$$


As noted in [1] p. 80 in connection with $z_{\min }$, we observe here in identical fashion that for $\phi \lesssim O(\theta)$ we have, in general, $z_{\operatorname{min\perp }} \lesssim 0\left(\theta^{2}\right)$ as originally assumed. (Since $\sigma_{1}=0$, we have $z_{\text {minl }}=0\left(\phi^{2}\right.$ ) from eq (VI.18).) Again as observed for eq (VII.9) in [1], so also in eq (VI.18) given here, the denominator is zero for $\phi=\phi_{01}$, so that this equation is clearly invalid for $\phi \geqq \phi_{01}$. In fact, as noted in [1], it becomes invalid for $\phi$ somewhat less than $\phi_{01}$. Once $\phi$ is sufficiently close to $\phi_{01}$ that $\phi_{01}^{2}-\phi^{2}$ is less than $0\left(\theta^{2}\right)$, then $z_{\text {minl }}$ is larger than $0\left(\theta^{2}\right)$, and the assumptions made in deriving eq (VI.18) no longer hold. As shown in [1], as $\phi$ approaches $\phi_{01}$ (from below), the position of the minimum, $z_{\text {minl }}$, increases from $O\left(\theta^{2}\right)$ to $O\left(\theta^{4 / 3}\right)$. When $\phi$ reaches the critical value $\phi_{c l}\left(\phi_{C l}<\phi_{01}\right)$, the minimum fuses with the second maximum of the cross section, becoming an inflection point. For $\phi>\phi_{c \perp}$ the cross section $d^{3} \sigma_{\perp}$ no longer has a minimum. For $\theta_{1}=1^{\circ}, \varepsilon_{1}=140 \mathrm{MeV}$, and $k=95 \mathrm{MeV}$ we find, from a careful computer analysis of the cross section as a function of $v$ and $\phi$ (see fig $6(a))$,

$$
\phi_{C \perp}=0.3645^{\circ}
$$

From eq (VI.15) we have, for these same values of $\theta_{1}, \varepsilon_{1}$, and $k$,

$$
\phi_{01}=0.4293^{\circ} .
$$


Having thus far examined in detail the extremum (the minimum) of the cross section $d^{3} \sigma_{1}$ in the region $z \leqq 0\left(\theta^{2}\right)$, we now look for extrema (also solutions of eq (VI.2)) of this cross section in the region $z=0(\theta)$. Again we follow the analys is given in [1], pp. 81-93. In contrast to the case $z=0\left(\theta^{2}\right)$, we now find two extrema. These correspond to the two maxima of the cross section $d^{3} \sigma_{1}$. In zeroth order, i.e., neglecting terms of relative $0(\theta)$ as well as those of $0\left(\theta^{2}\right)$, we have, directly from eq (VII.14) on p. 83 of [1],

$$
z_{\max 1}^{(0)}= \pm \frac{\delta\left(1+u^{2}\right)}{u}\left[1-\left(\frac{\phi}{\phi_{01}}\right)^{2}\right]^{\frac{1}{2}} .
$$

The full solution for the maxima of the cross section $d^{3} \sigma_{1}$ (i.e., with neglect only of terms of relative $0\left(\theta^{2}\right)$ ) is, from eq (VII.32) on p. 93 of $[1]$,

$z_{\operatorname{max\perp }}= \pm \frac{\delta\left(1+u^{2}\right)}{u}\left[1-\left(\frac{\phi}{\phi_{0 \perp}}\right)^{2}\right]^{\frac{1}{2}}+\frac{u f_{\perp}^{\prime}(u)}{4 f_{\perp}(u)} \frac{\delta^{2}\left(1+u^{2}\right)^{2}}{u^{2}}\left[1-\left(\frac{\phi}{\phi_{0 \perp}}\right)^{2}\right]-\frac{1}{2} \lambda_{\perp}{ }^{(2)}$.

Here $f_{\perp}(u)$ and $f_{\perp}^{\prime}(u)=\left.\frac{\partial f_{\perp}(v)}{\partial v}\right|_{v=u}$ are given by eq $(v .38)$, from which 


$$
\frac{f_{\perp}^{\prime}(u)}{f_{\perp}(u)}=-\frac{2 u}{1+u^{2}}
$$

and, from eqs (VII.20) and (VII.21) on p. 88 of [1], (and noting from eq (VI.7) that $\sigma_{1}=0$ ),

$$
\lambda_{\perp}{ }^{(2)}=\frac{3 g_{\perp}{ }^{\prime}(u)}{8 u f_{\perp}(u)} \phi^{2}-\frac{g_{\perp}(u) f_{\perp}^{\prime}(u)}{2 u f_{\perp}^{2}(u)} \phi^{2}-\frac{u f_{\perp}^{\prime}(u)}{2 f_{\perp}(u)}\left(\phi^{2}+\frac{\delta^{2}\left(1+u^{2}\right)^{2}}{u^{2}}\right) .
$$

As was the case for $z_{\text {minl }}$, derived under the assumption that $z=0\left(\theta^{2}\right)$, we note that here too the solution is valid only for $\phi<\phi_{01}$. And since we assume now that $z=0(\theta)$ we must again have

$$
\phi_{01}^{2}-\phi^{2}=0\left(\theta^{2}\right)
$$

or

$$
\phi_{0 \perp}-\phi=0(\theta)
$$

Again we see that the solutions given in eqs (VI.19) and (VI.20) break down as $\phi$ approaches $\phi_{01}$.

We have now found, for sufficiently small $\phi$, the minimum of the cross section $d^{3} \sigma_{1}$, given by eq (VI.18), and the two maxima, given by eq (VI.20), or, to zeroth order, by eq (VI.19). We see that the form of the cross section $d^{3} \sigma_{1}$ in the region of small momentum transfers 
$(q \lesssim O(u \theta))$ and its behavior in this region as a function of $v$ and $\phi$ are very similar to that of the cross section for unpolarized photons discussed in [1]. This similarity holds both in the region of the minimum $\left(z=0\left(\theta^{2}\right)\right)$ and in the region of the maxima (where $z=0(\theta)$ ) (see figs. $7(a)-7(d))$.

We now return to our consideration of the extrema of the cross section $d^{3} \sigma_{\|}$. Given the similarity of the expressions (VI.11) and (VI.12) for the positions of the minima for the cross sections $d^{3} \sigma_{\perp}$ and $d^{3} \sigma_{\|}$, it might be expected that the analysis just given for $d^{3} \sigma_{1}$, paralleling that presented in [1] for the cross section for unpolarized photons, could be repeated without modification for $d^{3} \sigma_{\|}$. The essential factor in that analysis, as may be seen from eqs (VI.13), (VI.16), and (VI.18), is the factor

$$
\frac{g_{1}(u)}{2 u^{2} f_{1}(u)}-1
$$

which appears in the denominator of $z_{\text {minl }}$ and in the numerator of $z_{\operatorname{max1}}$ (eqs (VI.19) and (VI.20)). However, implicit in the definition (VI.16) for $\phi_{\text {ol }}$ is the assumption that

$$
\frac{g_{\perp}(u)}{2 u^{2} f_{\perp}(u)}-1>0 \text {. }
$$

This is clearly true from eq (VI.15), and was also the case for $\frac{g(u)}{2 u^{2} f(u)}-1$ as given in [1] p. 82, eq. (VII.17a). However, the corresponding term for $d^{3} \sigma_{\|}$is, from eqs (VI.5) and (V.41), 


$$
\begin{aligned}
\frac{g_{\|}(u)}{2 u^{2} f_{\|}(u)}-1 & =\frac{2 k^{2}}{k^{2}+4 \varepsilon_{1} \varepsilon_{\dot{2}}\left(\frac{u^{2}-1}{u^{2}+1}\right)^{2}}-1 \\
& =\frac{k^{2}-4 \varepsilon_{1} \varepsilon_{2}\left(\frac{u^{2}-1}{u^{2}+1}\right)^{2}}{k^{2}+4 \varepsilon_{1} \varepsilon_{2}\left(\frac{u^{2}-1}{u^{2}+1}\right)^{2}} .
\end{aligned}
$$

Thus if

$$
k^{2}>4 \varepsilon_{1} \varepsilon_{2}\left(\frac{u^{2}-1}{u^{2}+1}\right)^{2} \equiv k_{0}^{2},
$$

we can again define, in analogy with eq (VI.16),

$$
\begin{aligned}
\phi_{\text {O\|l }} & =\frac{\delta\left(1+u^{2}\right)}{u}\left(\frac{g_{\|}(u)}{2 u^{2} f_{\|}(u)}-1\right)^{-\frac{1}{2}} \\
& =\frac{\delta\left(1+u^{2}\right)}{u}\left[\frac{k^{2}+4 \varepsilon_{1} \varepsilon_{2}\left(\frac{u^{2}-1}{u^{2}+1}\right)^{2}}{k^{2}-4 \varepsilon_{1} \varepsilon_{2}\left(\frac{u^{2}-1}{u^{2}+1}\right)^{2}}\right]^{\frac{1}{2}} .
\end{aligned}
$$


Then paralleling the steps followed in going from eq (VI.13) to eq (VI.18) we have, from eq (VI.5),

$$
g_{\|}^{\prime}(v)=\frac{16 k^{2} \varepsilon_{1} \varepsilon_{2} u}{\left(1+u^{2}\right)}\left(\frac{1-v^{2}}{\left(1+v^{2}\right)^{2}}\right),
$$

and thus, with $f_{\|}(v)$ as given by eq $(V .41)$,

$$
\frac{g_{\|}^{\prime}(u)}{u f_{\|}(u)}=4\left(\frac{1-u^{2}}{1+u^{2}}\right)\left(\frac{k^{2}}{k^{2}+4 \varepsilon_{1} \varepsilon_{2}\left(\frac{u^{2}-1}{u^{2}+1}\right)^{2}}\right) .
$$

Substituting eqs (VI.6), (VI.24), (VI.26), and (VI.27) in (VI.14) we may then write the expression for $z_{\text {minll }}$ in the form

$$
=\frac{\frac{1}{2}\left(\frac{u^{2}-1}{u^{2}+1}\right)\left[\left(k^{2}-4 \varepsilon_{1} \varepsilon_{2}\right) \phi^{2}-\frac{2 k\left(\varepsilon_{1}+\varepsilon_{2}\right)}{\varepsilon_{1} \varepsilon_{2}}\left(u^{2}+1\right)\right]\left(\phi^{2}+\frac{\delta^{2}\left(1+u^{2}\right)^{2}}{u^{2}}\right)+2 k^{2} \phi^{2}\left(\frac{\phi^{2}}{2}+\frac{\delta\left(1+u^{2}\right)}{\varepsilon_{2}}\right)}{\left[k^{2}-4 \varepsilon_{1} \varepsilon_{2}\left(\frac{u^{2}-1}{u^{2}+1}\right)^{2}\right]\left(\phi_{\text {oll }}^{2}-\phi^{2}\right)}
$$

The comments made in connection with $z_{\text {minl }}$, following eq (VI.18), are equally applicable to $z_{\text {minll }}$ for the present case in which the photon energy is sufficiently large that the inequality (VI.25) is satisfied. 
Thus we have, in general, $z_{\text {minll }} \lesssim O\left(\theta^{2}\right)$ as originally assumed. Further, the denominator in eq (VI.28) is zero for $\phi=\phi_{\text {oll }}$, so that the equation is clearly invalid for $\phi \geqq \phi_{\text {oll }}$. Again, as noted in [1], it becomes invalid for $\phi$ somewhat less than $\phi_{01}$. Once $\phi$ is sufficientiy close to $\phi_{01}$ that $\phi_{01}^{2}-\phi^{2}$ is less than $0\left(\theta^{2}\right)$, then $z_{\text {minll }}$ is larger than $O\left(\theta^{2}\right)$, and the assumptions made in deriving eq (VI.28) no longer hold. As $\phi$ approaches $\phi_{\text {oll }}$ (from below), the position of the minimum, $z_{\text {minll }}$, increases from $0\left(\theta^{2}\right)$ to $0\left(\theta^{4 / 3}\right)$. When $\phi$ reaches the critical value $\phi_{c l l}\left(\phi_{c l l}<\phi_{\text {oll }}\right)$, the minimum fuses with the second maximum of the cross section, becoming an inflection point. (see fig. $5(f)$. ) for $\phi>\phi_{c l l}$ the cross section $d^{3} \sigma_{\| l}$ no longer has a minimum. Again following the analysis given in [1], pp. 81-93 for the extrema of the cross section in the region $z=0(\theta)$, we now find two extrema, corresponding to the two maxima of the cross section $d^{3} \sigma_{\|}$, as we just did for $d^{3} \sigma_{\perp}$ (eqs (VI.10) and (VI.20)). In zeroth order, i.e., neglecting terms of relative $O(\theta)$ as well as those of $0\left(\theta^{2}\right)$, we have, directly from eq (VII.14) on p. 83 of [1],

$$
z_{\max \|}^{(0)}= \pm \frac{\delta\left(1+u^{2}\right)}{u}\left[1-\left(\frac{\phi}{\phi_{0 \| l}}\right)^{2}\right]^{\frac{1}{2}}
$$

The full solution for the maxima of the cross section $d^{3} \sigma_{\|}$(i.e., with neglect only of terms of relative $0\left(\theta^{2}\right)$ ) is, from eq (VII.32) on p. 93 of [1], 
$z_{\max \|}= \pm \frac{\delta\left(1+u^{2}\right)}{u}\left[1-\left(\frac{\phi}{\phi 0_{\|}}\right)^{2}\right]^{\frac{1}{2}}+\frac{u f_{\|}^{\prime}(u)}{4 f_{\| 1}(u)} \frac{\delta^{2}\left(1+u^{2}\right)^{2}}{u^{2}}\left[1-\left(\frac{\phi}{\phi_{\text {oll }}}\right)^{2}\right]-\frac{1}{2} \lambda_{\|}^{(2)}$.

Here $f_{\| l}(u)$ and $f_{\|\|}^{\prime}(u)=\left.\frac{\partial f_{\| I}(v)}{\partial v}\right|_{v=u}$ are given by eq (v.41), from which

$$
\frac{f_{\| 1}^{\prime}(u)}{f_{\| I}(u)}=-\frac{2 u}{1+u^{2}}\left[\frac{k^{2}-4 \varepsilon_{1} \varepsilon_{2}\left(\frac{u^{2}-1}{u^{2}+1}\right)^{2}}{k^{2}+4 \varepsilon_{1} \varepsilon_{2}\left(\frac{u^{2}-1}{u^{2}+1}\right)^{2}}\right],
$$

and, from eqs (VII.20) and (VII.21) on p. 88 of [1],

$\lambda_{\|}{ }^{(2)}=\frac{3 \sigma_{\|}}{u}+\frac{3 g_{\|}{ }^{\prime}(u)}{8 u f_{\|}(u)} \phi^{2}-\frac{g_{\|}(u) f_{\|}{ }^{\prime}(u)}{2 u f_{\|}{ }^{2}(u)} \phi^{2}-\frac{u f_{\| \prime}{ }^{\prime}(u)}{2 f_{\|\|}(u)}\left(\phi^{2}+\frac{\delta^{2}\left(1+u^{2}\right)^{2}}{u^{2}}\right)$.

As was the case for $z_{\text {minll }}$, derived under the assumption that $z=0\left(\theta^{2}\right)$, we note that here too the solution is valid only for $\phi<\phi_{\text {oll }}$. And since we assume now that $z=0(\theta)$ we must again have

$$
\phi_{\text {oll }}^{2}-\phi^{2}=0\left(\theta^{2}\right)
$$

or

$$
\phi_{\text {oll }}-\phi=0(\theta) \text {. }
$$


We have now found, for sufficiently small $\phi$, and assuming the condition (VI.25), the minimum of the cross section $d^{3} \sigma_{\|}$, given by eq (VI.28), and the two maxima, given by eq (VI.30), or, to zeroth order, by eq (VI.29). We see that, assuming the condition (VI.25), the form of the cross section $d^{3} \sigma_{\|}$in the region of small momentum transfers $(q \lesssim O(u \theta))$ and its behavior in this region as a function of $v$ and $\phi$ are very similar to that of the cross section $d^{3} \sigma_{1}$ as well as to that of the cross section for unpolarized photons discussed in [1]. This similarity holds both in the region of the minimum $\left(z=0\left(\theta^{2}\right)\right)$ and in the region of the maxima (where $z=0(\theta)$ ). (See figs. $8(a)-8(d)$.)

We have considered the cross section $d^{3} \sigma_{\|}$assuming the condition (VI.25) and noted its similarity to the cross section $d^{3} \sigma_{\perp}$ as well as to the cross section summed over polarizations, $d^{3} \sigma$, under this condition. We now consider the case in which (VI.25) does not hold, viz., when

$$
k^{2}<4 \varepsilon_{1} \varepsilon_{2}\left(\frac{u^{2}-1}{u^{2}+1}\right)^{2} \equiv k_{0}^{2} .
$$

It should be noted that for most situations of experimental interest, the condition (VI.33) is the more likely to occur: In general we have

$$
u=p_{1} \sin \theta_{1} \approx p_{1} \theta_{1} \gg 1
$$

Thus

$$
\left(\frac{u^{2}-1}{u^{2}+1}\right)^{2} \approx 1
$$


From (VI.33) we then have, approximately,

$$
k^{2}<4 \varepsilon_{1} \varepsilon_{2}=4 \varepsilon_{1}\left(\varepsilon_{1}-k\right)
$$

from which

$$
\left(k+2 \varepsilon_{1}\right)^{2}<8 \varepsilon_{1}^{2}
$$

or

$$
\frac{k}{\varepsilon_{1}}<2(\sqrt{2}-1) \approx 0.8
$$

Thus, if $p_{1} \theta_{1} \gg 1$, then for most of the spectrum $\left(0<\frac{k}{\varepsilon_{1}}<0.8\right)$ the condition (VI.33) holds. In this case, we see from eq (VI.24) that

$$
\frac{g_{\|}(u)}{2 u^{2} f_{\|}(u)}-1=\frac{k^{2}-4 \varepsilon_{1} \varepsilon_{2}\left(\frac{u^{2}-1}{u^{2}+1}\right)^{2}}{k^{2}+4 \varepsilon_{1} \varepsilon_{2}\left(\frac{u^{2}-1}{u^{2}+1}\right)^{2}}<0 .
$$

We can not then define $\sigma_{\text {oll }}$ as in eq (VI.26). We may, however, retain all of the expressions for $z_{\operatorname{minll}}$ and $z_{\operatorname{maxll}}$ if we now define 


$$
\begin{aligned}
\bar{\phi}_{\text {oll }} & =\frac{\delta\left(1+u^{2}\right)}{u}\left(1-\frac{g_{\|}(u)}{2 u^{2} f_{\| 1}(u)}\right)^{-\frac{1}{2}} \\
& =\frac{\delta\left(1+u^{2}\right)}{u}\left[\frac{4 \varepsilon_{1} \varepsilon_{2}\left(\frac{u^{2}-1}{u^{2}+1}\right)^{2}+k^{2}}{4 \varepsilon_{1} \varepsilon_{2}\left(\frac{u^{2}-1}{u^{2}+1}\right)^{2}-k^{2}}\right],
\end{aligned}
$$

The expression (VI.28) for $z_{\text {minll }}$ is then modified only in that the term $\phi_{\text {oll }}^{2}$ must be replaced by $-\bar{\phi}_{\text {oll }}^{2}$. The numerator of (VI.28) is thus unchanged, and the term $\phi_{\text {oll }}^{2}-\phi^{2}$ in the denominator is replaced by

$$
-\left(\bar{\phi}_{\text {oll }}^{2}+\phi^{2}\right)
$$

In the region of small momentum transfers $(q \lesssim 0(u \theta)$, and thus also $\phi \lesssim 0(\theta)$ ) we now find $z_{\text {minll }}$ of $0\left(\theta^{2}\right)$ for all $\phi$; the denominator does not become smaller as $\phi$ increases and the modified expression for $z_{\text {minll }}$ does not break down at some critical angle. Similarly, the expressions (VI.29) and (VI.30) for $z_{\text {maxll }}$ need only be modified by replacing $\phi_{\text {oll }}^{2}$ by $-\bar{\phi}_{\text {oll }}^{2}$. The modified expressions for $z_{\max \|}$ are then of $O(\theta)$ for all $\phi$; they do not break down at some critical angle. In summary, then, for photon energies such that the condition (VI.33) holds, the cross section $d^{3} \sigma_{\|}$has, for al1 $\phi$, one minimum in the region $z=0(\theta)$, provided, of course, that we remain in the region of sma11 momentum transfers. This difference in the behavior of $d^{3} \sigma_{\|}$ and $d^{3} \sigma_{1}$ for photon energies satisfying the condition (VI.33), shown 
in figs. $9(a)-9(d)$, is clearly significant for the polarization: For $\phi>\phi_{c \perp}, d^{3} \sigma_{\perp}$ has no sharp dip, as may be seen in figs. $g(c)$ and $g(d)$, whereas $d^{3} \sigma_{\|}$has a sharp dip regardless of the values of $\phi$. (We are of course still assuming that $\phi$ is small, of $0(\theta)$, so that we are still in the region of small momentum transfers.) Then in this region of the dip in $d^{3} \sigma_{\|}$the polarization, defined in eq (II.1), is very large, i.e., the tagged photons are highly polarized perpendicular to the production plane (see figs. $3(a)-3(h)$ ). 
VII. COULOMB CORRECTIONS TO THE DIFFERENTIAL CROSS SECTIONS FOR POLARIZED BREMSSTRAHLUNG

The analysis presented in this report thus far has dealt exclusively with the first Born approximation differential cross sections for polarized bremsstrahlung, $\mathrm{d} \sigma_{1}$ and $d \sigma_{\|}$. In particular we have considered the behavior of these cross sections in the region of small momentum transfers.

As noted in [1], Sec. IX, the Coulomb correction to the differential cross section for bremsstrahlung at high energies has been calculated specifically in this region of small momentum transfers. In connection with the cross section for unpolarized photons it was first given for a pure Coulomb field (no screening) by Bethe and Maximon [18], and later for arbitrary screening by 01 sen, Maximon, and Wergeland [19]. In both cases it was found that the entire effect of the Coulomb correction is simply to multiply the Born approximation cross section by a factor which, al though it varies throughout the region of small momentum transfers, is always of order unity. This work was extended to the case of polarized photons by 01 sen and Maximon [16], who showed that the same multiplicative factor applies to each of the completely differential cross sections for polarized photons, $d^{3} \sigma_{1}$ and $d^{3} \sigma_{\|}$. This factor, denoted by $|A|^{2}$ in [19] and by $R$ in [18], is given for the case of no screening on $p .123$ of [1]. Thus also for the cross sections $d^{3} \sigma_{\perp}$ and $d^{3} \sigma_{\|}$, the Coulomb corrections do not fill in the sharp dips, as they might if they were additive. Moreover, since the same multiplicative factor applies to both of the completely differential 
cross sections, $d^{3} \sigma_{1}$ and $d^{3} \sigma_{\|}$, it does not enter in the polarization, $P$, eq (II.1). However, once we integrate over some region of angles, as is required in the analysis of most experiments, Coulomb corrections do indeed enter the expression for the polarization.

It must be recalled that in the analysis presented in references [18], [19], and [16], there have been neglected throughout, not only terms of relative order $1 / \varepsilon^{2}$, as in the present report, but terms of relative order $l / \varepsilon$ as well. Thus if one wishes to evaluate the Coulomb corrections to the differential cross section to the same level of accuracy as that pursued here for the Born approximation, the analyses given earlier in [18], [19], and [16] must be extended to include the terms of relative order $l / \varepsilon$. 


\section{REFERENCES}

[1] Leonard C. Maximon, Arlette de Miniac, and Thierry Anie1, Tagged Photons, An analysis of the bremsstrahlung differential cross section in the range of interest for a tagged photon system, National Bureau of Standards Report NBSIR 81-2262, Apri1 1981.

[2] Nuclear Physics Research with Microtrons Using Superconducting Linacs. July 1980 Report of the Nuclear Physics Lab., University of Illinois, Urbana, I11. (unpublished).

[3] Ahrens, J., Borchert, H., Czock, K. H., Eppler, H. B., Gimm, H., Gundrum, H., Kröning, M., Riehn, P., Sita Ram. G., Zieger, A., and Ziegler, B., Nuc1. Phys. A251, 479 (1975).

Borchert, H., Ziegler, B., Gimm, H., Zieger, A., Hughes, R., and Ahrens, J., Description du double spectromètre Compton du MPI de Mayence, Comptes Rendus des journées d'études de Saclay, 14-18 mars 1977.

[4] Arends, J., Eyink, J., Hartmann, H., Hegerath, A., Mecking, B., Nöldeke, G., and Rost, H., The tagged photon beam at the $500 \mathrm{MeV}$ synchrotron at Bonn, Comptes Rendus des journées d'études de Saclay, 14-18 mars 1977. This material is also available as a report of the Physikalisches Institut of Bonn University, preprint no. BONN-HE-78-19 (Apri1 1979).

[5] Christophe Tzara, Comptes Rendus Acad. Sci. 239, 44 (1954).

[6] Jacques Miller, Polarization of bremsstrahlung, CEN Saclay Note CEA-655, 1957.

[7] D. Jamnik and P. Axe], Phys. Rev. 117, 194 (1960). This article also gives other references to ear $\overline{1 i e r}$ experiments.

[8] Katsufusa Shoda, A new arrangement for experiments using polarized bremsstrahlung, L.A.L. Report 1135, Apri1 1965 (Laboratoire de 1 'Accélérateur Linéaire, Université de Paris, B.P. 5, Orsay, France).

[9] F. F. Liu, Phys. Rev. 138, B1443 (1965).

[10] A. M. Nathan, R. Starr, R. M. Laszewski, and P. Axe1, Phys. Rev. Lett. $\underline{42}, 221$ (1979). 
[11] K. Wienhard, R. Schneider, K. Ackermann, K. Bangert, U. E. P. Berg, G. Junghans, W. Naatz, and R. Stock, Photonuclear reactions with linearly polarized photons, in Abstracts of contributed papers to the International Conference on Nuclear Physics with Electromagnetic Interactions, Mainz, Germany, June 5-9, 1979.

K. Ackermann, K. Bangert, U. E. P. berg, K. Kobras, W. Naatz, R. Schneider, R. Stock, and K. Wienhard, pp. 3-9 of Tätigkeitsbericht 1979, Kernphysik, Strahlenzentrum, Justus-Liebig-Universität Giessen, D-63 Giessen, West Germany.

K. Ackermann, K. Bangert, U. E. P. Berg, C. Bläsing, K. Kobras, W. Naatz, D. Rück, R. K. M. Schneider, R. Stock, and K. Wienhard, Large bound El-strength in $T=1$ sd-shell nuclei, p. 274 of Vol. 1 Abstracts of the Proceedings of the International Conference on Nuclear Physics, August 24-30, 1980, Berkeley, California.

K. Wienhard, R. K. M. Schneider, K. Ackermann, K. Bangert, U. E. P. Berg, and R. Stock, A study of ${ }^{16} 0(\gamma, p)^{15} \mathrm{~N}$ with 1 inearly polarized photons, p. 47 of Workshop on nuclear physics with real and virtual photons, from collective states to quarks in nuclei, Noyember 25-28, 1980.

U. E. P. Berg, K. Ackermann, K. Bangert, C. Bläsing, K. Kobras, K. Naatz, D. Rück, R. K. M. Schneider, R. Stock, and K. Wienhard, Nuclear resonance fluorescence measurements with linearly polarized bremsstrahlung photons, p. 49 of Workshop on nuclear physics with real and virtual photons, from collective states to quarks in nuclei, November 25-28, 1980.

[12] Michael M. May, Phys. Rev. 84, 265 (1951). Note that in eq (1) on p. 266 of this article the sign of the term with factor $q^{2}$ is incorrect -- it should be negative.

[13] R. L. Gluckstern, M. H. Hu11, Jr., and G. Breit, Science 114, 480 (1951); Phys. Rev. 90, 1026 (1953).

[14] H. Kolbenstyedt and Haakon 01sen, Nuovo Cimento 22, 610 (1961); note Sec. 2 and Appendix A.

[15] Haakon 01sen and L. C. Maximon, Nuovo Cimento 24, 186 (1962).

[16] Haakon 01sen and L. C. Maximon, Phys. Rev. 114, 887 (1959); note Eq (4.9), p. 892, which does not contain any correlations between two momenta and only one polarization.

[17] Haakon 01sen and L. C. Maximon, Phys. Rev. Lett. 13, 112 (1964).

[18] H. A. Bethe and L. C. Maximon, Phys. Rev. 93, 768 (1954).

[19] Haakon 01sen, L. C. Maximon, and Harald Wergeland, Phys. Rev. 106, 27 (1957). 


\section{FIGURE CAPTIONS}

Fig. 1 Momenta of the initial and final electrons, $\mathrm{p}_{1}$ and $\mathrm{p}_{2}$, and photon momentum and polarization, $\underline{k}$ and $\underline{e}$, in the system with $z$-axis in the direction of $\underline{k}$, and $\underline{p}_{1}$ in the $x-z$ plane.

Fig. 2(a) The cross section for unpolarized photons, $\frac{1}{z^{2}} d \sigma$ $\left(=\frac{1}{z^{2}} d^{3} \sigma / d \Omega_{p_{2}} d \Omega_{k} d k\right.$, eq (IV.1), p. 22 of [1]), and the perpendicular and parallel components of the momentum transfer, $q_{1}$ and $q_{2}$, as functions of $\theta_{2}$ for $\varepsilon_{1}=140 \mathrm{MeV}$, $k=95 \mathrm{MeV}, \theta_{1}=1^{\circ}$, and $\phi=0^{\circ}$.

2(b) The same as in 2(a), except for the cross section, which is for photons polarized perpendicular to the production plane, $\frac{1}{z^{2}} d \sigma_{1}\left(=\frac{1}{z^{2}} d^{3} \sigma_{1} / d \Omega_{p_{2}} d \Omega_{k} d k\right.$, eq (I.2), p.3).

2(c) The same as in 2(a), except for the cross section, which is for photons polarized parallel to the production plane, $\frac{1}{z^{2}} d \sigma_{\|}\left(=\frac{1}{z^{2}} d^{3} \sigma_{\|} / d \Omega_{p_{2}} d \Omega_{k} d k\right.$, eq (I.3), p.4).

Fig. 3(a) The polarization, $P=\left(d \sigma_{\perp}-d \sigma_{\|}\right) /\left(d \sigma_{\perp}+d \sigma_{\|}\right)$as a function of $\theta_{2}$, for $\varepsilon_{1}=140 \mathrm{MeV}, k=95 \mathrm{MeV}, \theta_{1}=1^{\circ}$, and several values of $\phi$, viz., $\phi=0^{\circ}, 0.07^{\circ}, 0.02^{\circ}, 0.03^{\circ}$, and $0.1^{\circ}$.

3(b) The same as in 3(a), except that $\phi=0^{\circ}, 0.5^{\circ}$, and $1^{\circ}$. 
Fig. 3(c) The same as in $3(\mathrm{a})$, except that $\phi=2^{\circ}, 6^{\circ}$, and $10^{\circ}$.

3(d) The same as in 3(a), except that $\phi=7^{\circ}$ and $30^{\circ}$.

3(e) The same as in 3(a), except that $k=30 \mathrm{MeV}$.

$3(f)$ The same as in $3(b)$, except that $k=30 \mathrm{MeV}$.

$3(\mathrm{~g})$ The same as in $3(\mathrm{c})$, except that $k=30 \mathrm{MeV}$.

$3(\mathrm{~h})$ The same as in $3(\mathrm{~d})$, except that $k=30 \mathrm{MeV}$.

Fig. 4(a) The cross section, $\frac{1}{z^{2}} d \sigma_{1}\left(=\frac{1}{z^{2}} d^{3} \sigma_{1} / d \delta_{p_{2}} d \Omega_{k} d k\right)$, for photons polarized perpendicularly to the production plane, as

a function of $\theta_{2}$, for $k<k_{0}$. (From eq (VI.25), $k_{0}^{2}=4 \varepsilon_{1} \varepsilon_{2}\left(\left(u^{2}-1\right)\left(\left(u^{2}+1\right)\right)^{2}\right.$.) We choose $\varepsilon_{7}=140 \mathrm{MeV}$, $k=95 \mathrm{MeV}, \theta_{1}=1^{\circ}$, and $\phi=0^{\circ}, 0.25^{\circ}, 0.5^{\circ}$, and $0.75^{\circ}$.

4 (b) The same as $4(a)$, except that $k=130 \mathrm{MeV}$, so that $k>k_{0}$, and $\phi=0^{\circ}, 2^{\circ}, 4^{\circ}$, and $6^{\circ}$.

4(c) The same as $4(a)$, except that we plot the cross section, $\frac{1}{z^{2}} d \sigma_{\|}\left(=\frac{1}{z^{2}} d^{3} \sigma_{\|} / d \delta \varepsilon_{p_{2}} d s z_{k} d k\right)$, for photons polarized para17el to the production plane.

4(d) The same as 4(b), except that we plot the cross section, $\frac{1}{z^{2}} d \sigma_{\|}\left(=\frac{1}{z^{2}} d^{3} \sigma_{\|} / d \Omega \varepsilon_{p_{2}} d \Omega \Omega_{k} d k\right)$, for photons polarized paral1e1 to the production prane.

Note that following the individual figures $4(a)-4(d)$ we present these four figures on a single page for easy comparison. 
Fig. 5(a) The positions, $z=(v-u) / u$, of the maxima, the minimum, and the points of inflection of the cross section $\frac{1}{z^{2}} d \sigma_{1} \quad\left(=\frac{1}{z^{2}} d^{3} \sigma_{1} / d \Omega_{p_{2}} d \Omega_{k} d k\right)$ as a function of the azimuthal angle $\phi$, for $k<k_{0}$. We choose $\varepsilon_{1}=140 \mathrm{MeV}, k=95 \mathrm{MeV}$, and $\theta_{1}=1^{\circ}$.

5 (b) The same as 5(a), except that $k>k_{0}$. (We choose $k=130 \mathrm{MeV}$. ) Note that in this figure the ranges of $\phi$ and $z$ are $0^{\circ} \leqq \phi \leqq 40^{\circ}$, and $-1.0 \leqq z \leqq 0.2$

$5(c)$ The same as 5(b), except that the ranges of $\phi$ and $z$ are $0^{\circ} \leqq \phi \leqq 10^{\circ}$ and $-0.2 \leqq z \leqq 0.2$.

5(d) The same as 5(a), except that we consider the cross section $\frac{1}{z^{2}} d \sigma_{\|}\left(=\frac{1}{z^{2}} d^{3} \sigma_{\|} / d \Omega_{p_{2}} d \Omega_{k} d k\right)$.

$5(e)$ The same as $5(b)$, except that we consider the cross section $\frac{1}{z^{2}} d \sigma_{\|} \quad\left(=\frac{1}{z^{2}} d^{3} \sigma_{\|} / d \Omega_{p_{2}} d \Omega_{k} d k\right)$.

$5(f)$ The same as $5(\mathrm{c})$, except that we consider the cross section $\frac{1}{z^{2}} d \sigma_{\|} .\left(=\frac{1}{z^{2}} d^{3} \sigma_{\|} / d \Omega_{p_{2}} d \Omega_{k} d k\right)$.

NOTE: Only in Fig. 5(d), pertaining to the cross section do with $k<k_{0}$, do the minimum and second maximum remain as $\phi$ increases. 
Fig. $6(a)$ The positions, $z=(v-u) / u$, of the minimum and the second maximum of the cross section $\frac{1}{z^{2}} d \sigma_{1}\left(=\frac{1}{z^{2}} d^{3} \sigma_{1} / d \Omega_{p_{2}} d \Omega_{k} d k\right)$ as a function of the azimuthal angle, $\phi$, for $k<k_{0}$. Here $\varepsilon_{1}=140 \mathrm{MeV}, k=95 \mathrm{MeV}$, and $\theta_{1}=1^{\circ}$.

$6(b)$ The same as in $6(a)$, except that $k>k_{0}$. (We choose $k=130 \mathrm{MeV}$ ).

6 (c) The same as in $6(\mathrm{~b})$, except that we consider the cross section $\frac{1}{z^{2}} d \sigma_{\|}\left(=\frac{1}{z^{2}} d^{3} \sigma_{\|} / d \Omega_{p_{2}} d \Omega_{k} d k\right)$.

Fig. $7(a)$ The cross sections $\frac{1}{z^{2}} d \sigma\left(=\frac{1}{z^{2}} d^{3} \sigma / d \Omega_{p_{2}} d \Omega_{k} d k\right)$ and $\frac{1}{z^{2}} d \sigma_{1}\left(=\frac{1}{z^{2}} d^{3} \sigma_{1} / d \Omega_{p_{2}} d \Omega_{k} d k\right)$ as functions of $\theta_{2}$, for $\varepsilon_{1}=140 \mathrm{MeV}, k=95 \mathrm{MeV}, \theta_{1}=1^{\circ}$, and $\phi=0^{\circ}$.

7 (b) The same as in $7(a)$, except that $\phi=0.2^{\circ}$.

7 (c) The same as in $7(a)$, except that $\phi=0.4^{\circ}$.

$7(d)$ The same as in 7(a), except that $\phi=0.6^{\circ}$. Note that following the individual figures $7(a)-7(d)$ we present these four figures on a single page for easy comparison.

Fig. 8(a) The cross sections $\frac{1}{z^{2}} d \sigma_{1}\left(=\frac{1}{z^{2}} d^{3} \sigma_{1} / d \Omega_{p_{2}} d \Omega_{k} d k\right)$ and $\frac{1}{z^{2}} d \sigma_{\|}\left(=\frac{1}{z^{2}} d^{3} \sigma_{\|} / d \Omega_{p_{2}} d \Omega_{k} d k\right)$ as functions of $\theta_{2}$, for $k>k_{0}$. Here $\varepsilon_{1}=140 \mathrm{MeV}, k=130 \mathrm{MeV}, \theta_{1}=7^{\circ}$, and $\phi=0^{\circ}$.

$8(b)$ The same as in $8(a)$, except that $\phi=0.2^{\circ}$.

$8(c)$ The same as in $8(a)$, except that $\phi=0.4^{\circ}$. 
Fig. 8(d) The same as in $8(a)$, except that $\phi=0.6^{9}$.

Note that following the individual figures $8(a)-8(d)$ we present these four figures on a single page for easy comparison.

Fig. $9(a)$ The cross sections $\frac{1}{z^{2}} d \sigma_{1}\left(=\frac{1}{z^{2}} d^{3} \sigma_{1} / d \Omega_{p_{2}} d \Omega_{k} d k\right)$ and $\frac{1}{z^{2}} d \sigma_{\|}\left(=\frac{1}{z^{2}} d^{3} \sigma_{\|} / d \Omega_{p_{2}} d \Omega_{k} d k\right)$ as functions of $\theta_{2}$, for $k<k_{0}$. Here $\varepsilon_{1}=140 \mathrm{MeV}, k=95 \mathrm{MeV}, \theta_{1}=1^{\circ}$, and $\phi=0^{\circ}$.

9 (b) The same as in $9(a)$, except that $\phi=0.2^{\circ}$.

9 (c) The same as in $9(a)$, except that $\phi=0.4^{\circ}$.

9 (d) The same as in $9(a)$, except that $\phi=0.6^{\circ}$. Note that following the individual figures $9(a)-9(d)$ we present these four figures on a single page for easy comparison.

NOTE: As illustrated also in fig. 5(d) for $k<k_{0}$, the minimum and second maximum for $d \sigma_{\|}$remain as $\phi$ increases. 


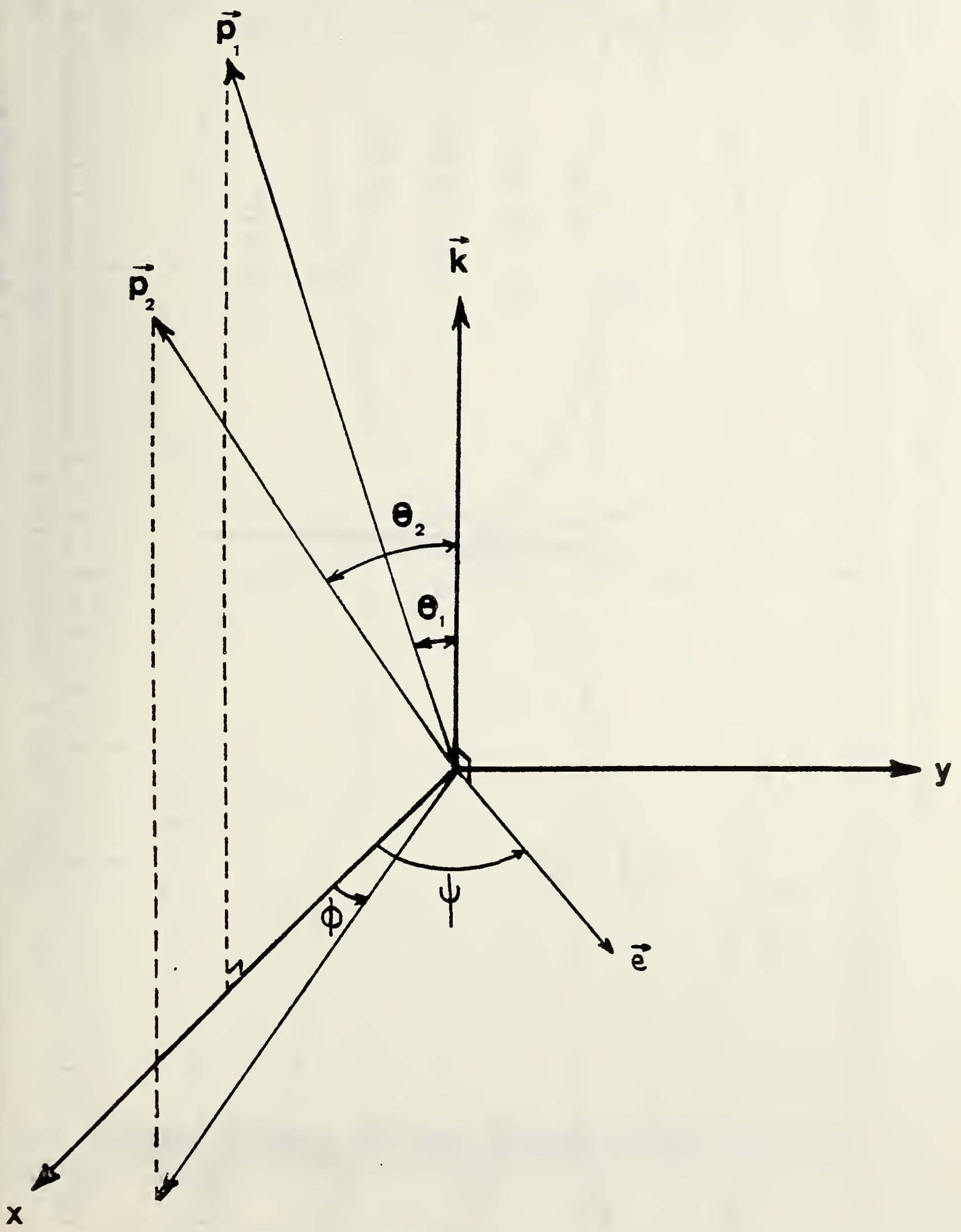

FIG. 1 


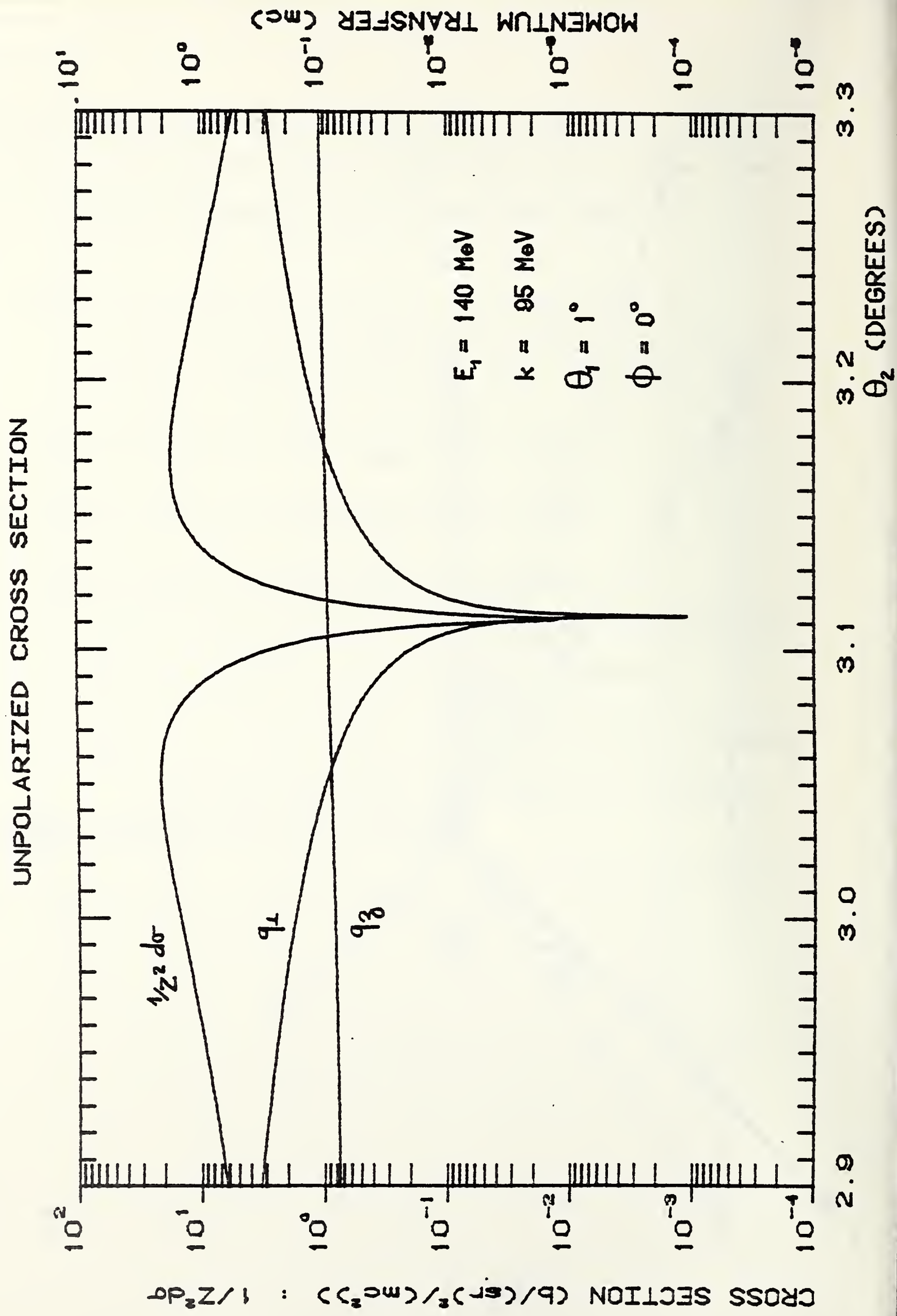




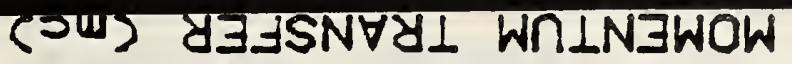

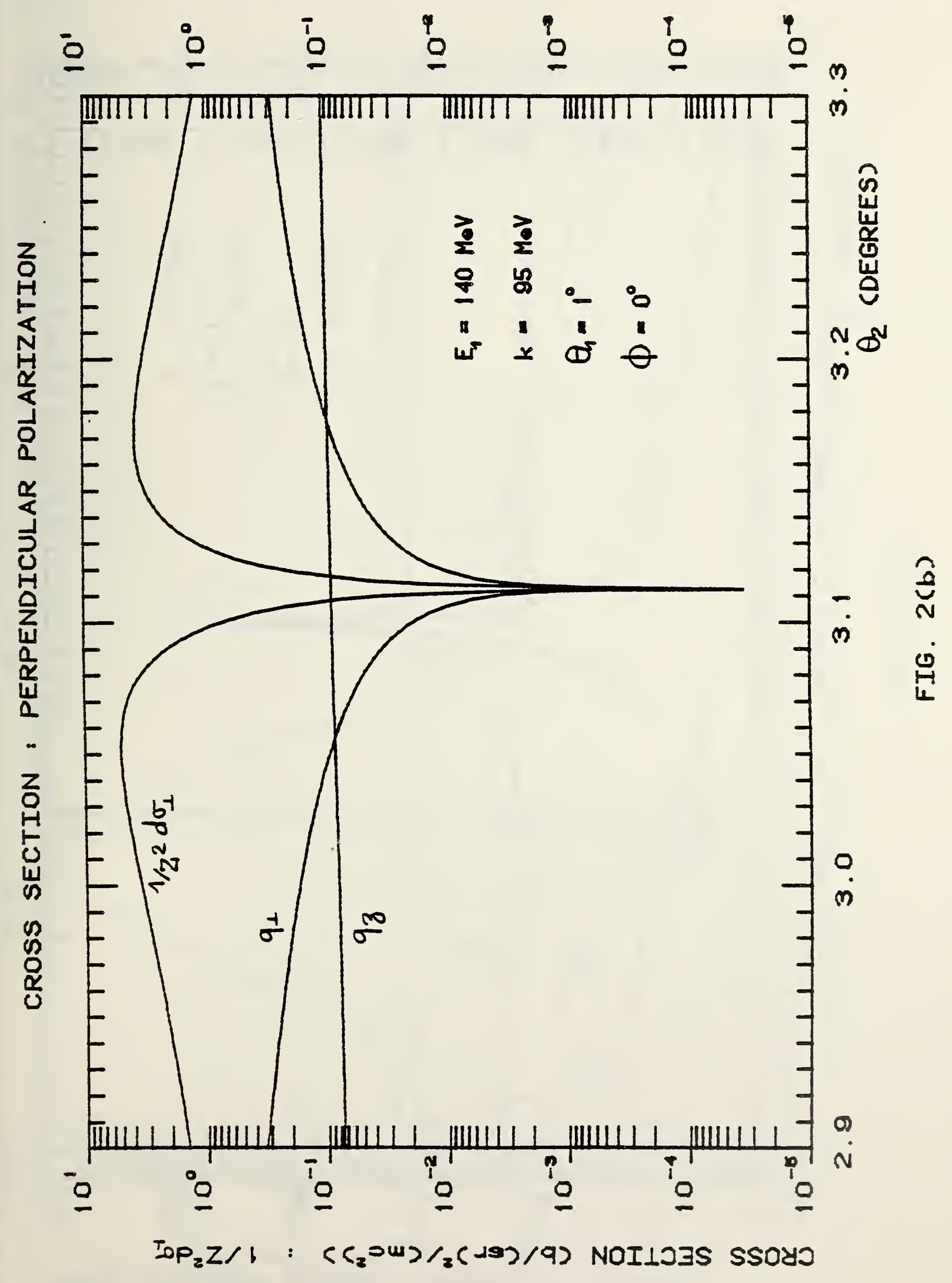




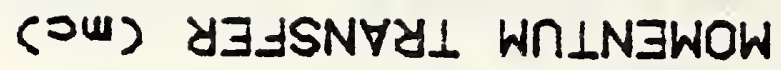

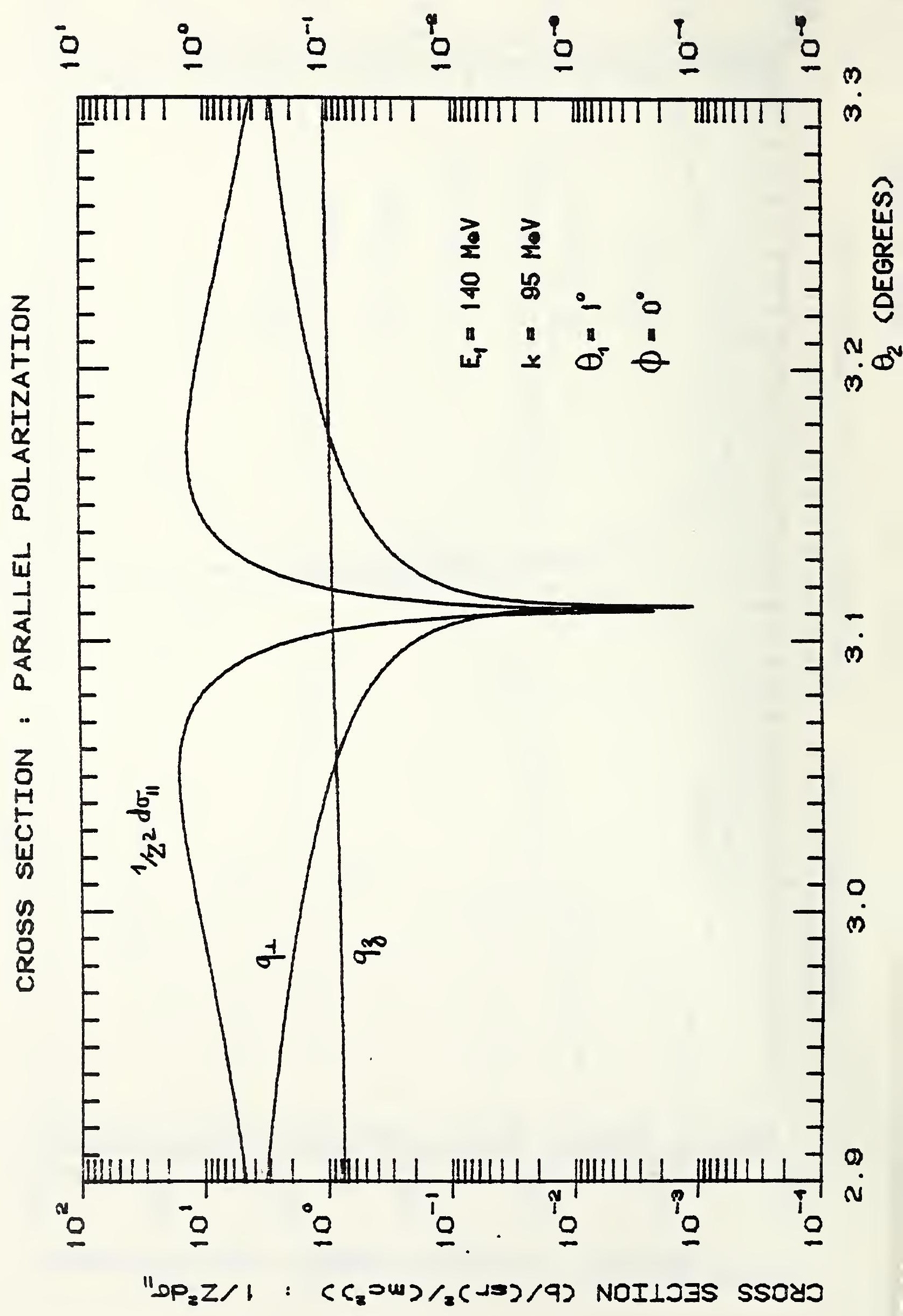




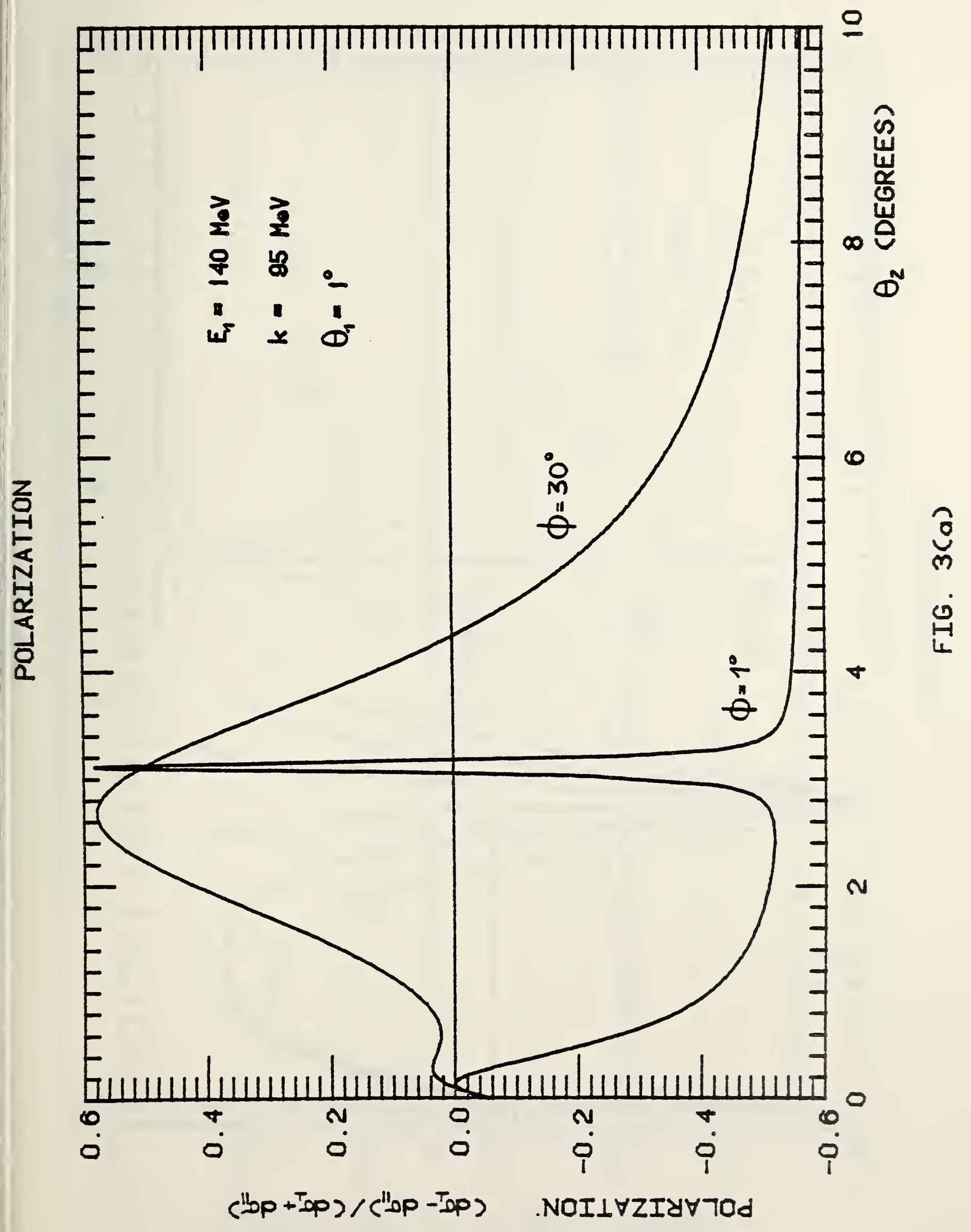




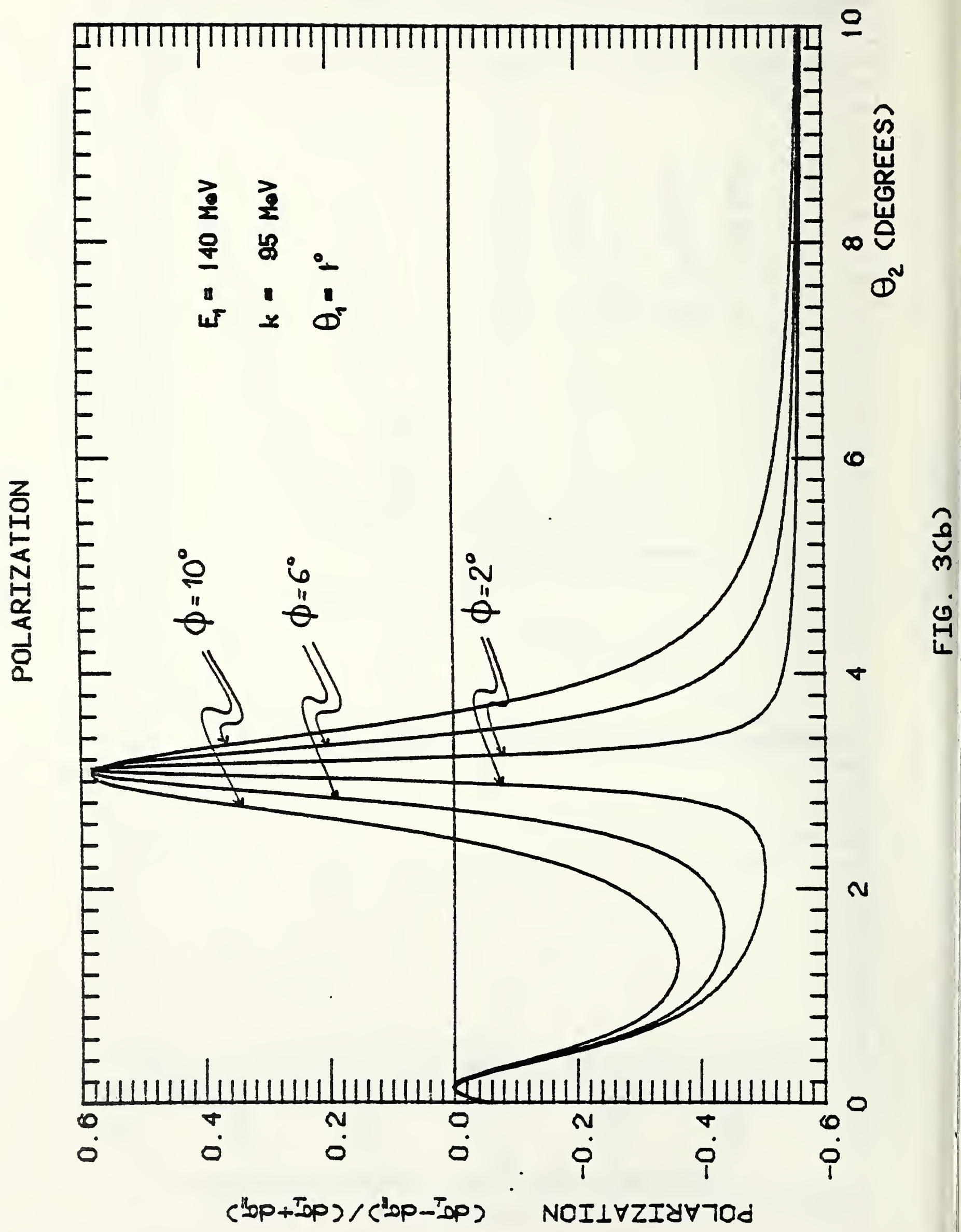




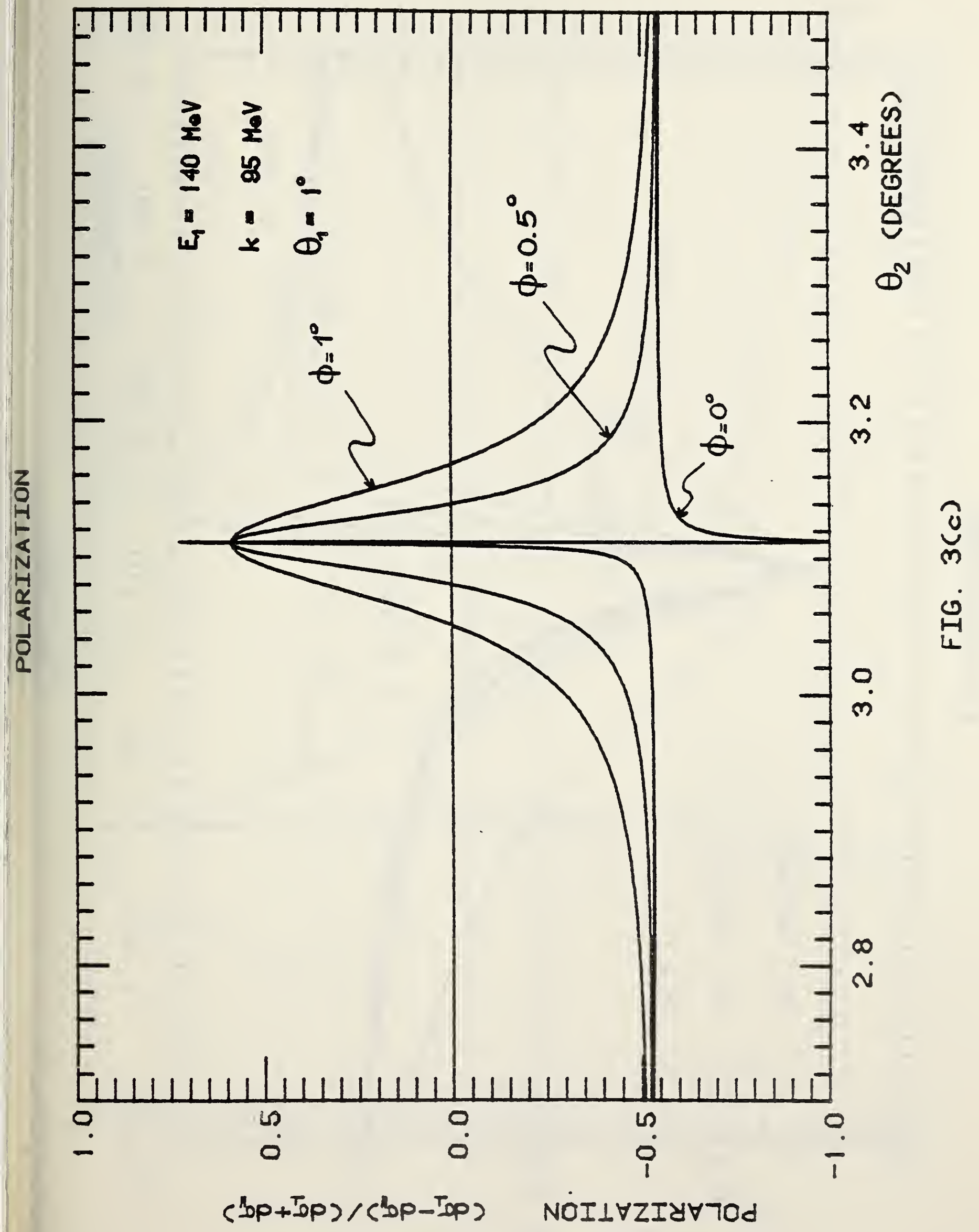




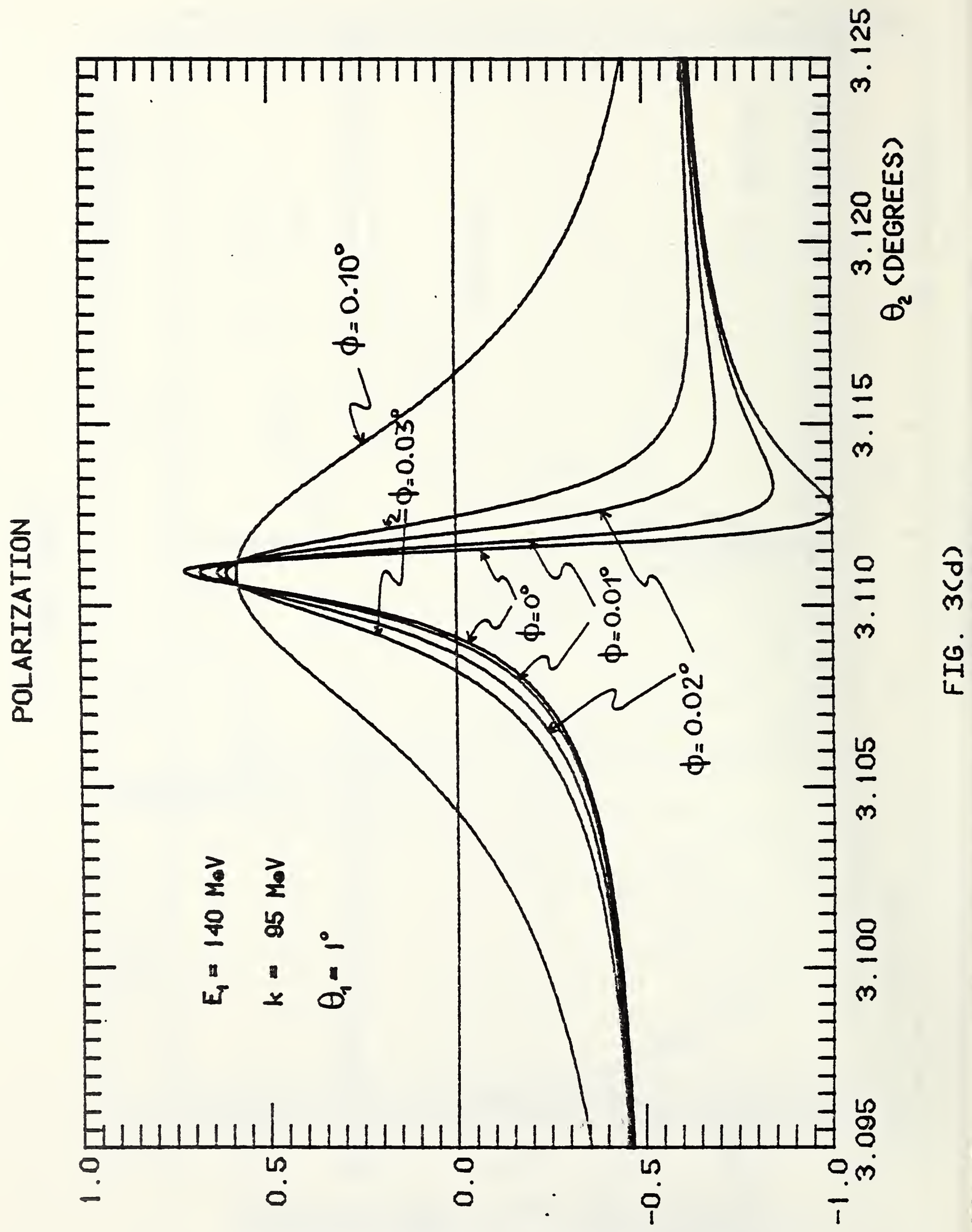

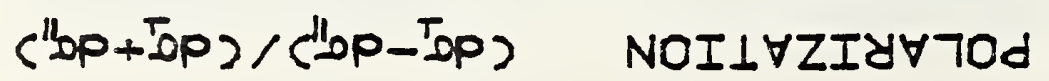




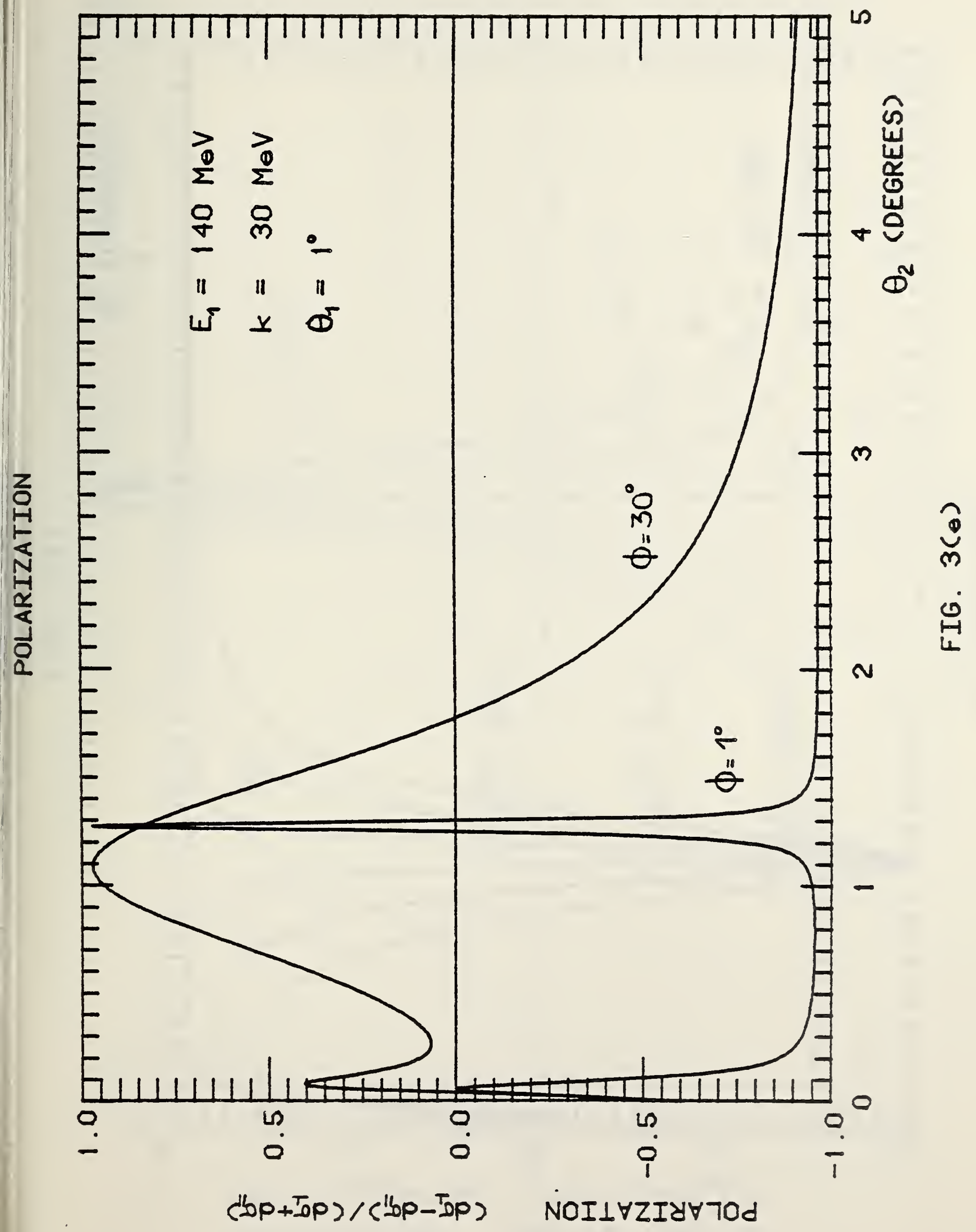




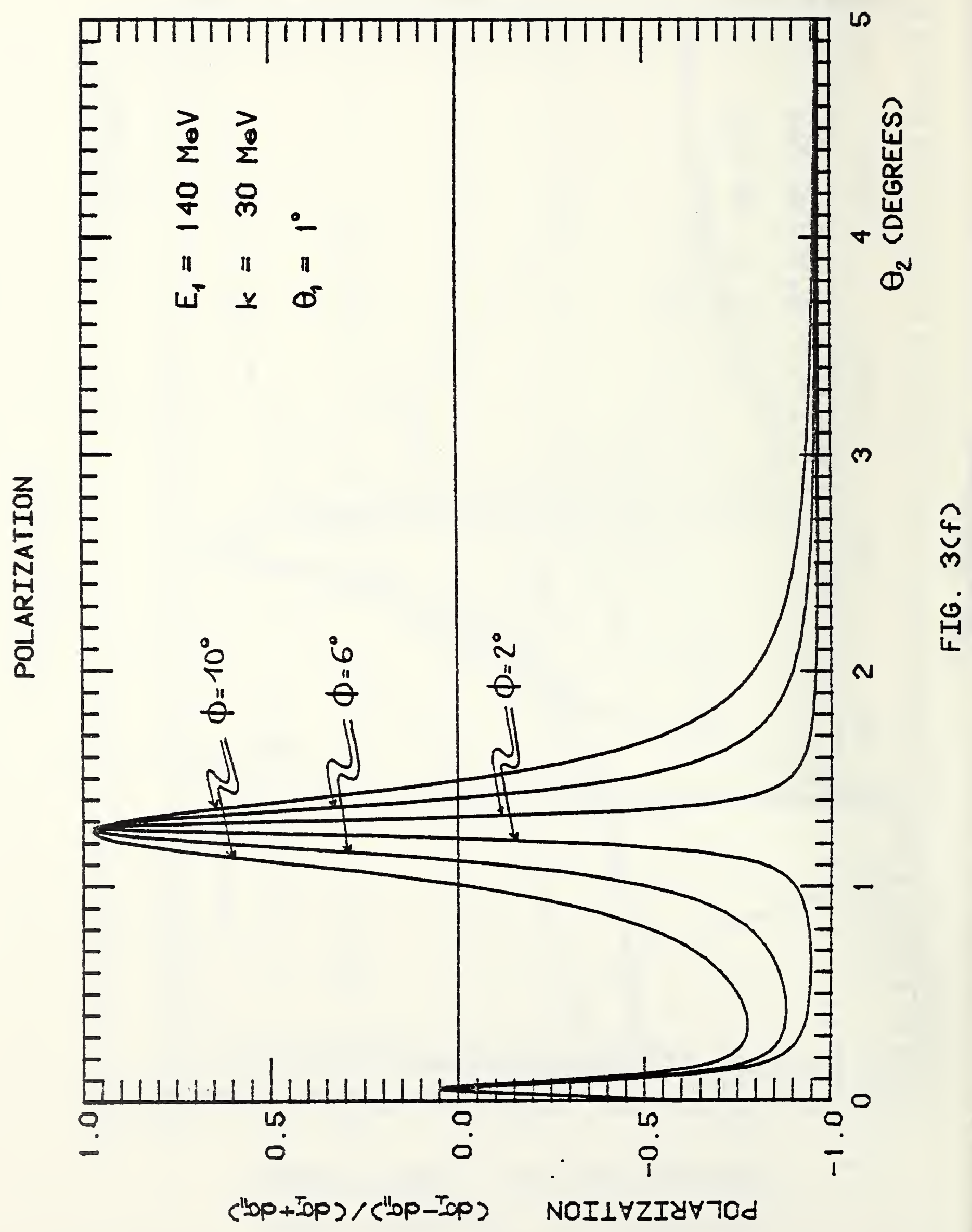




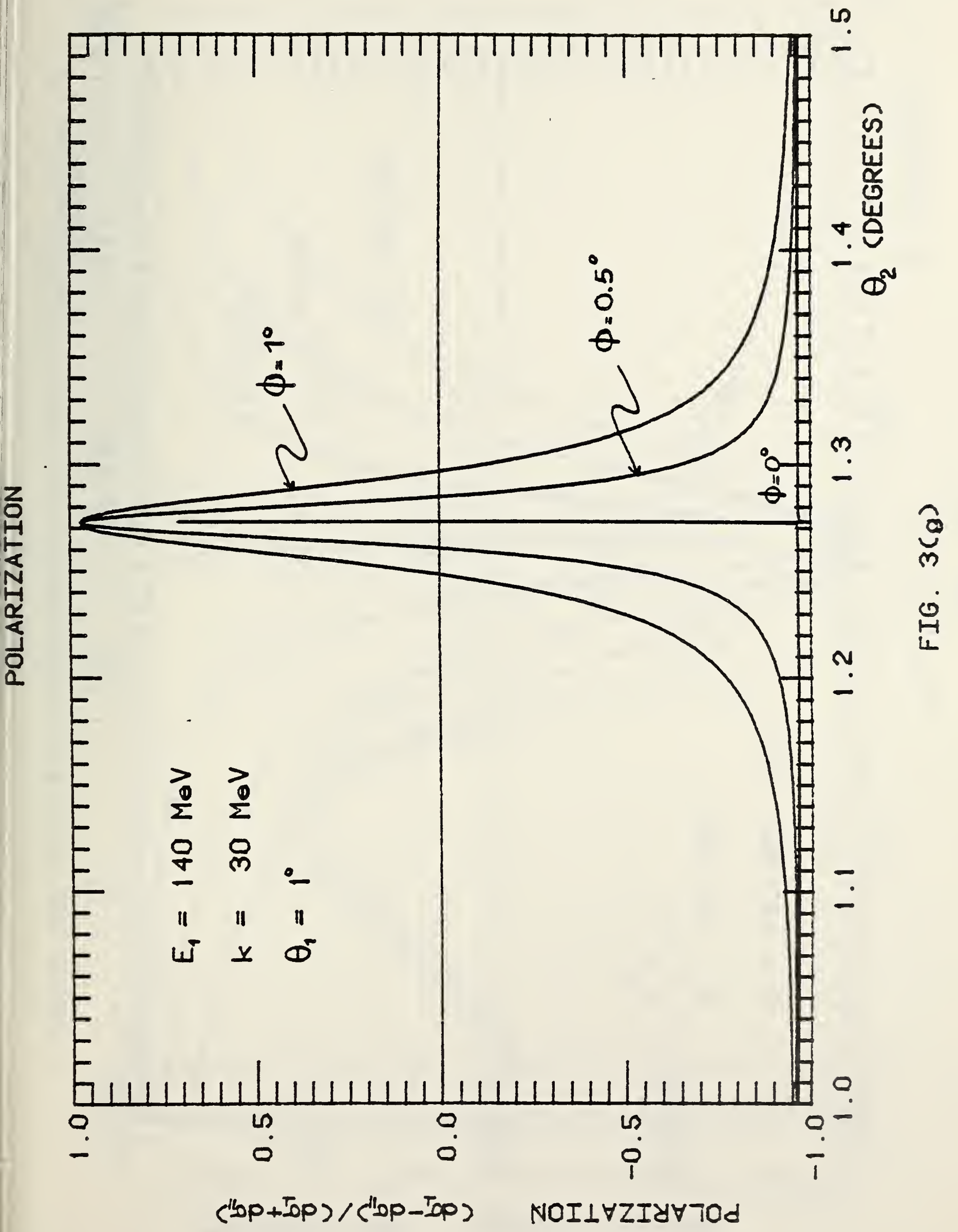




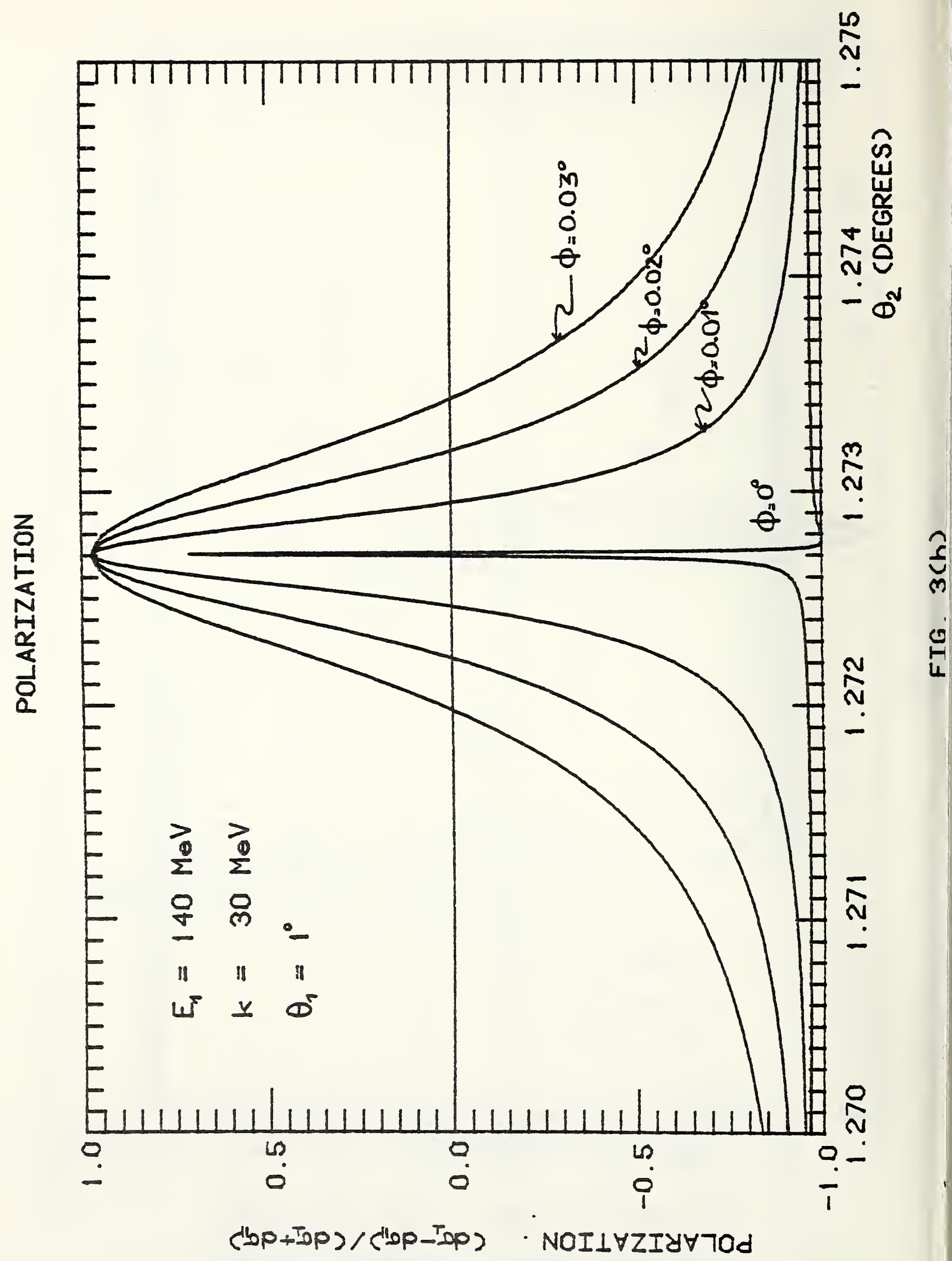




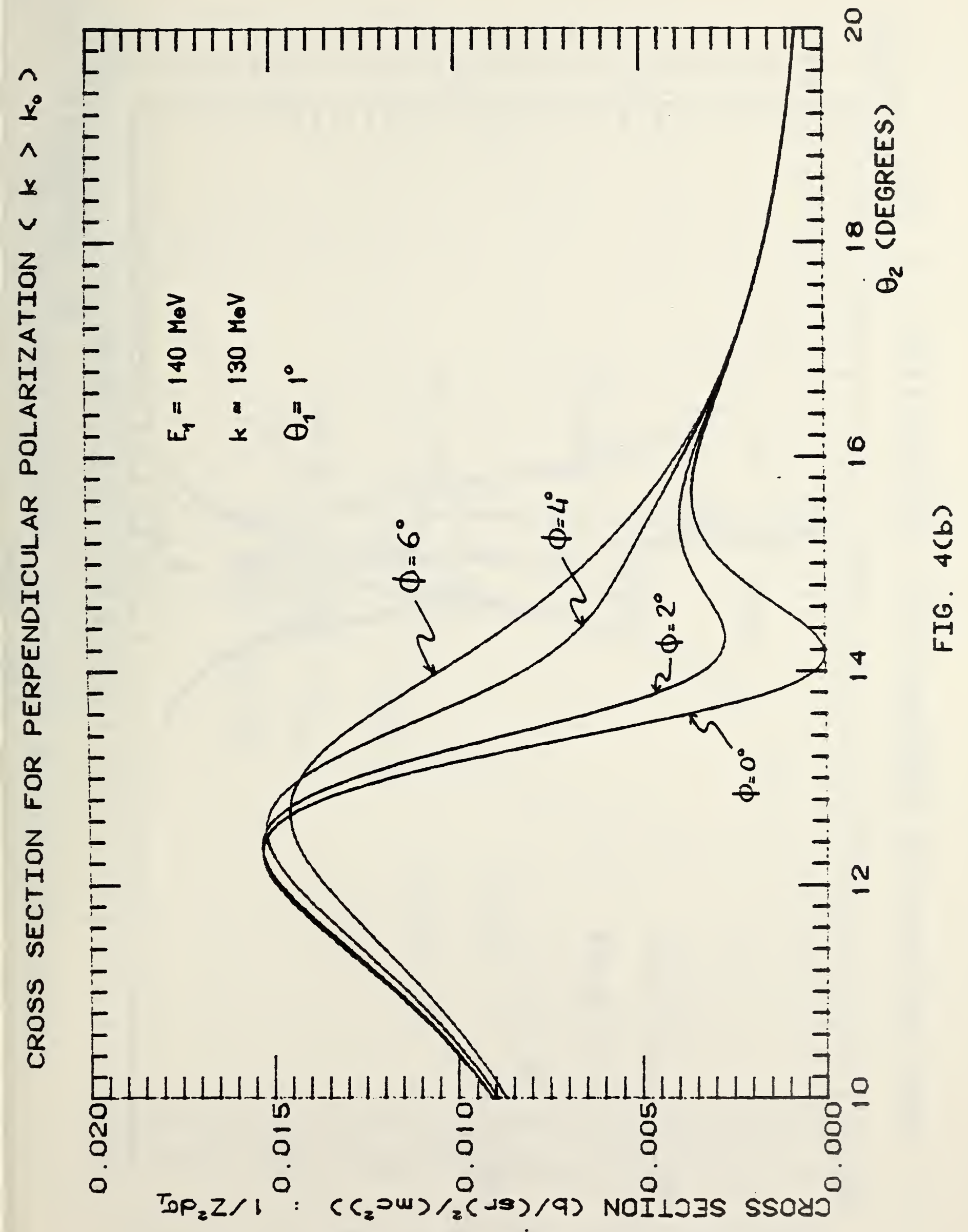




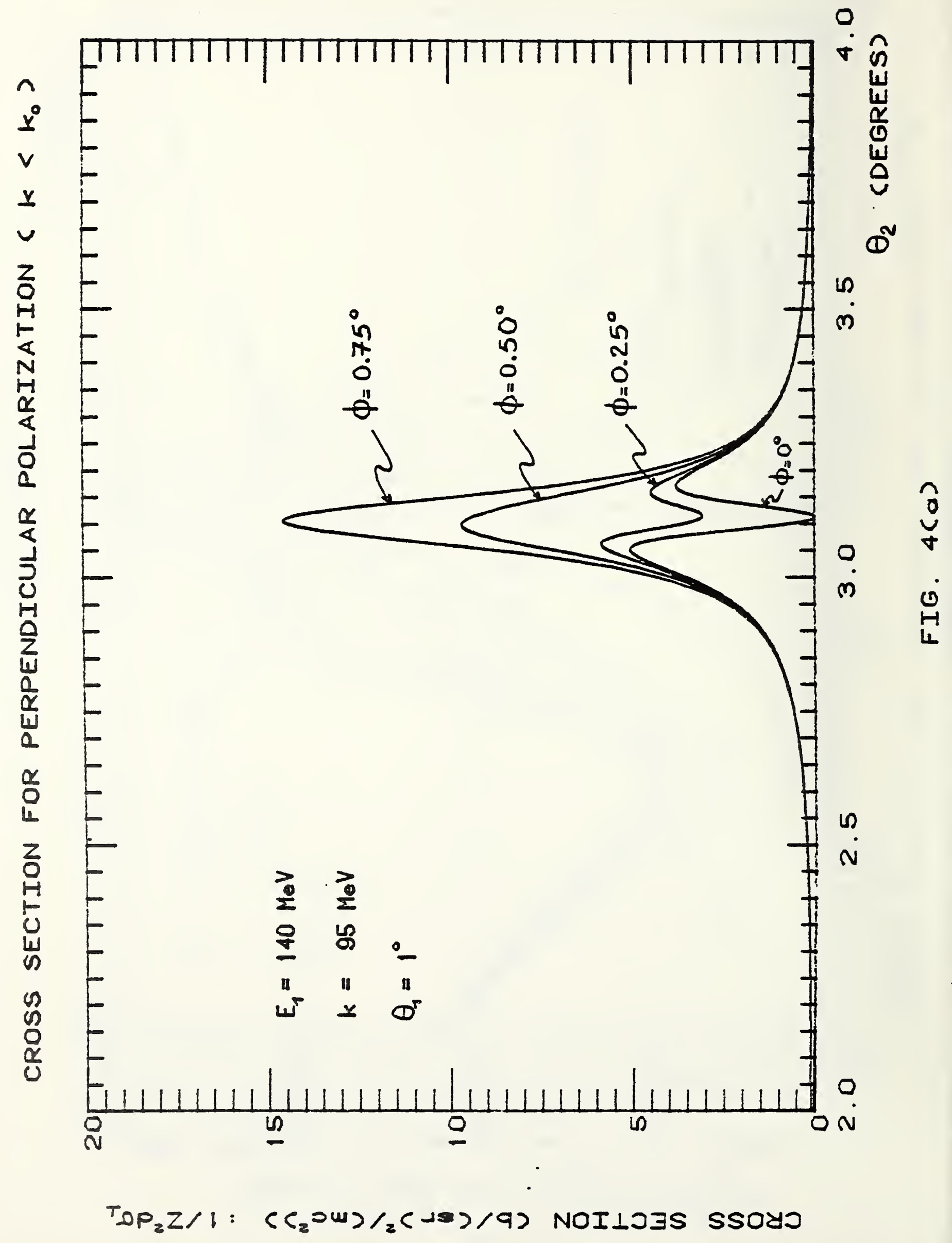




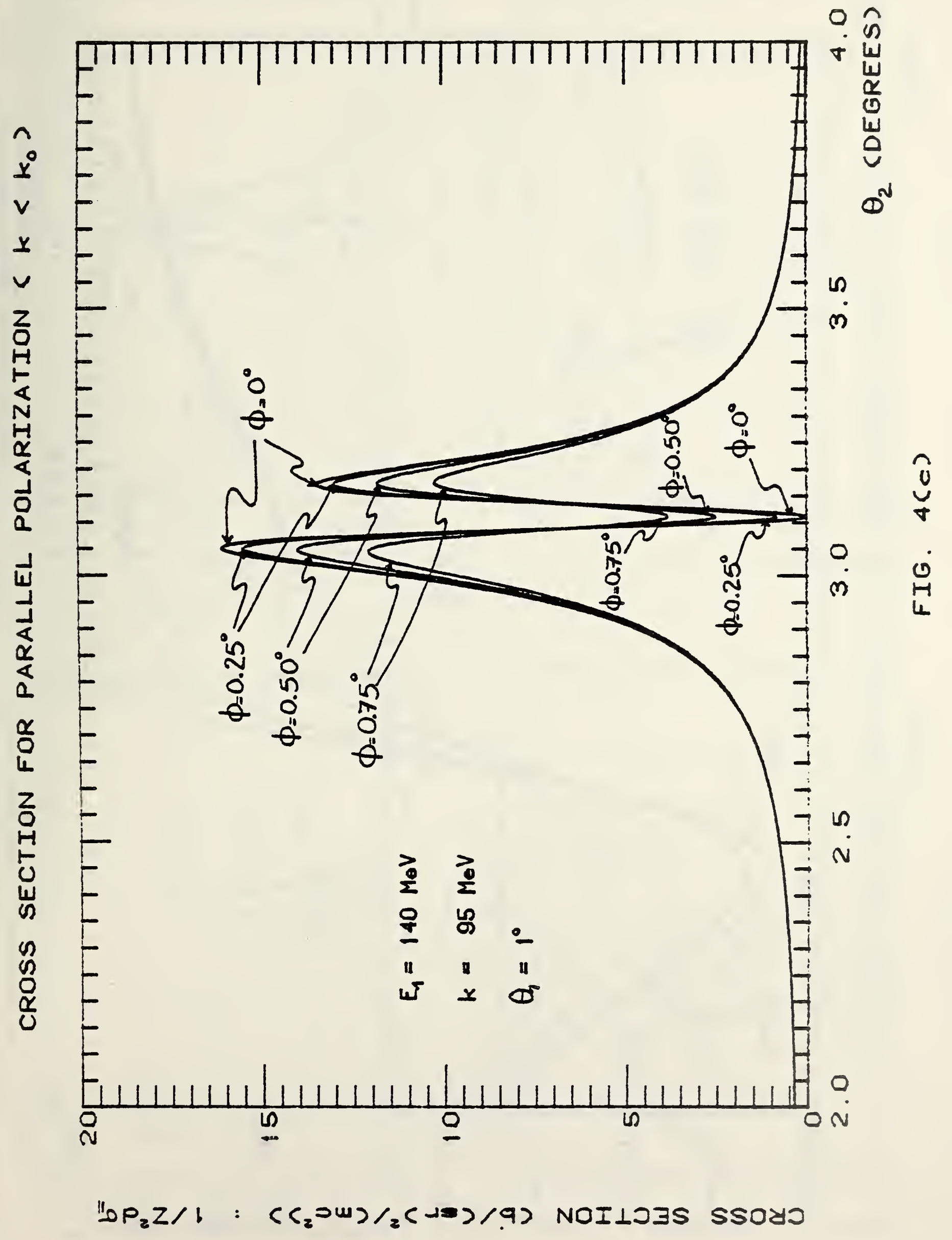




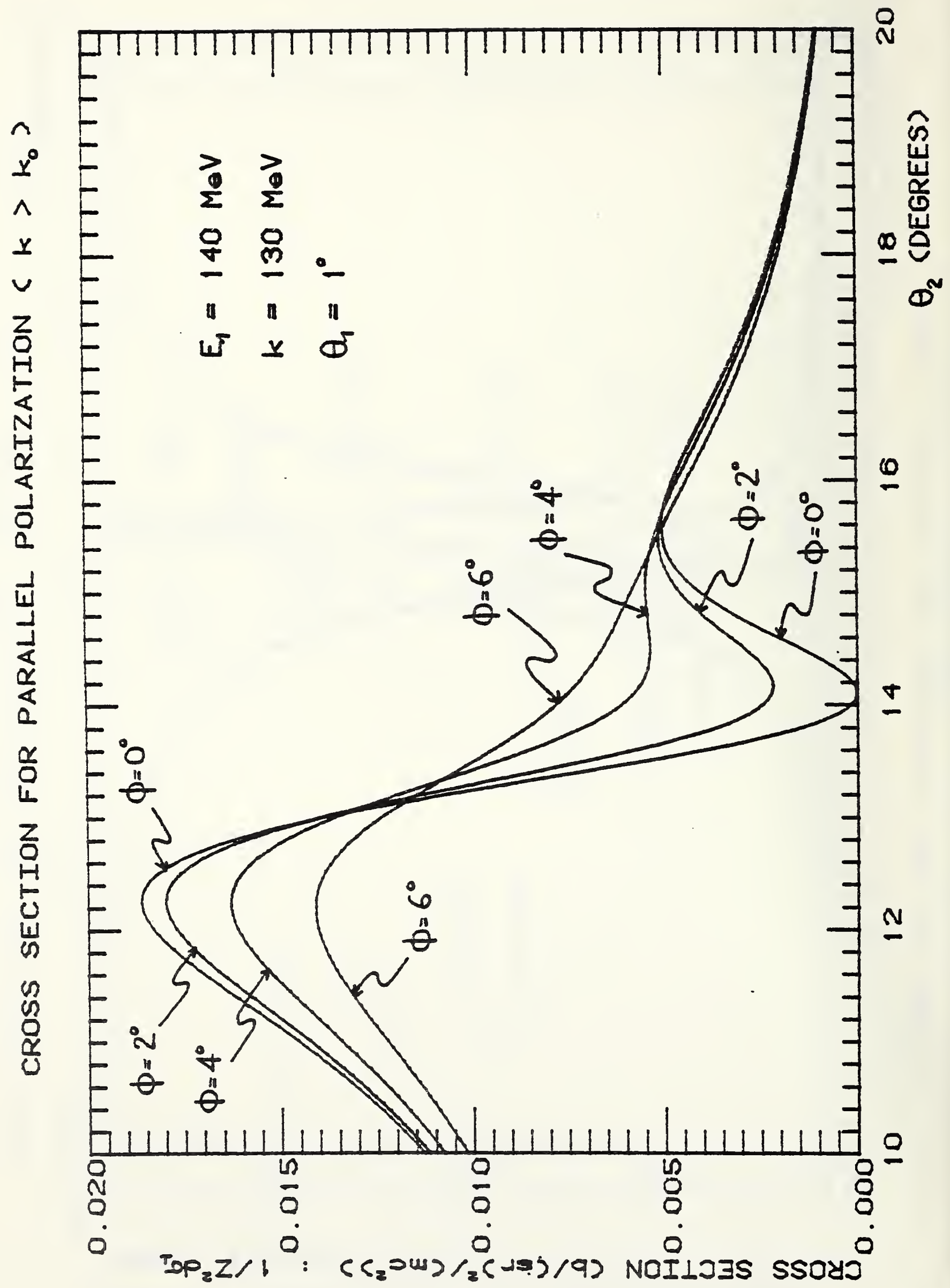




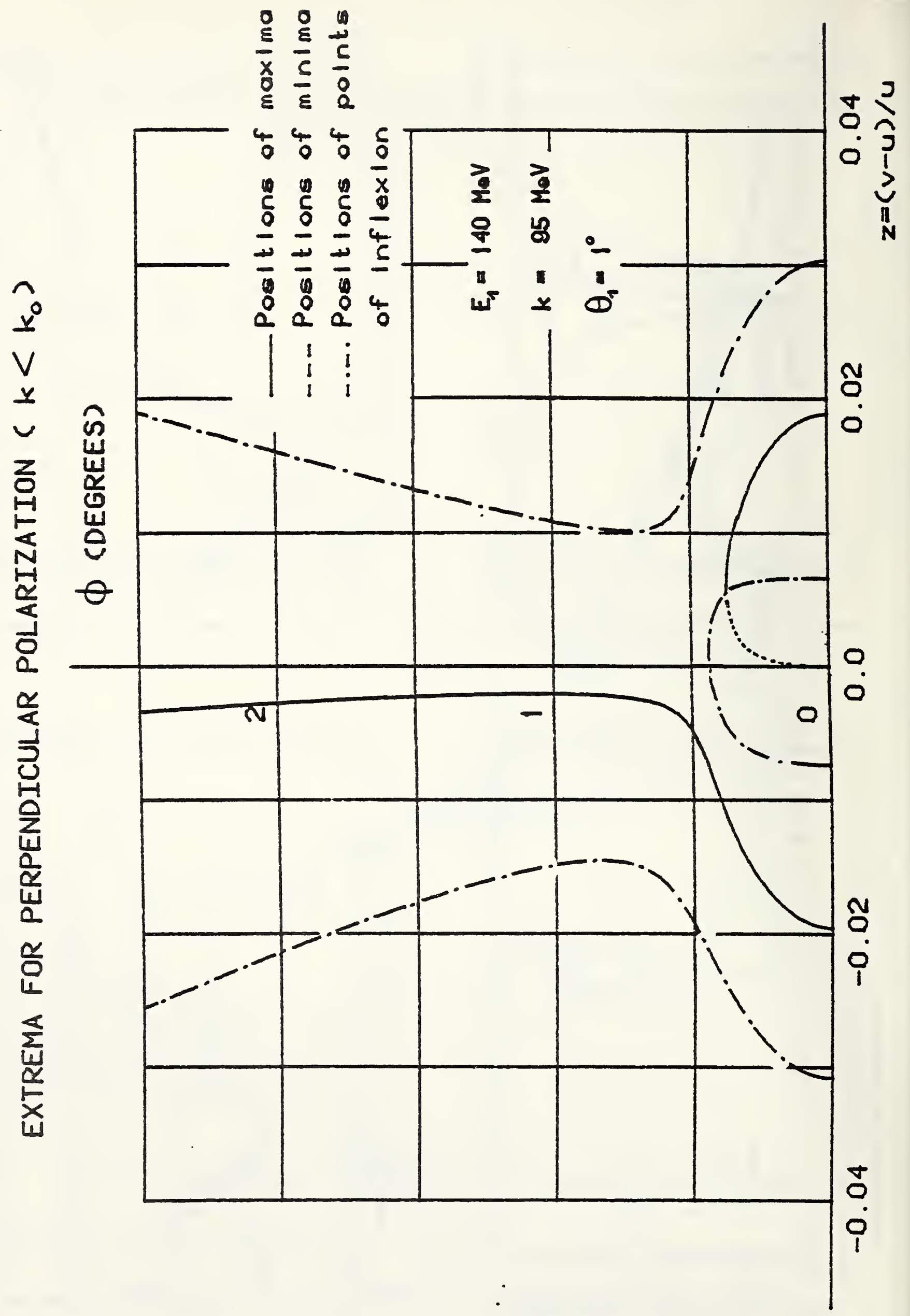




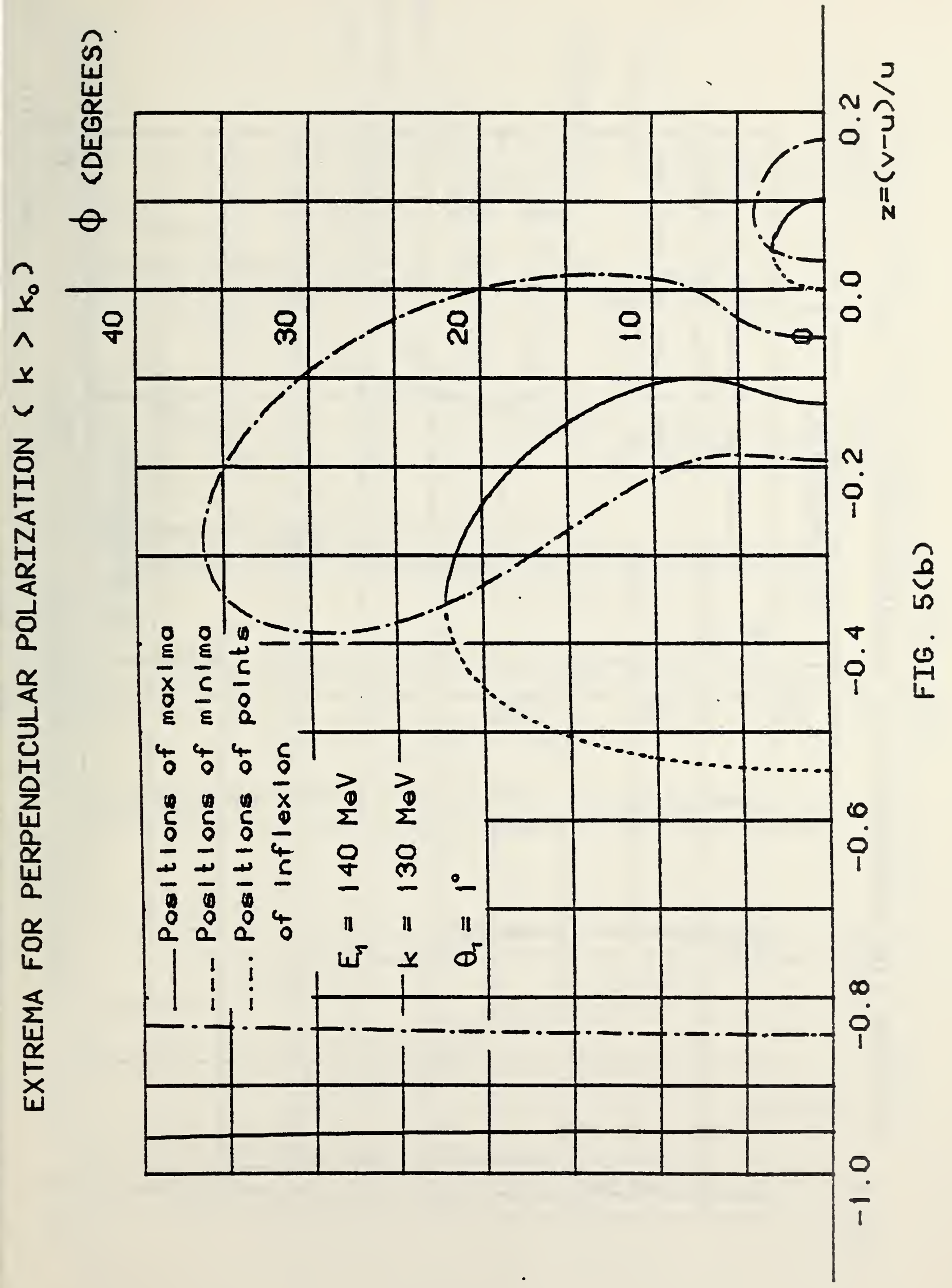




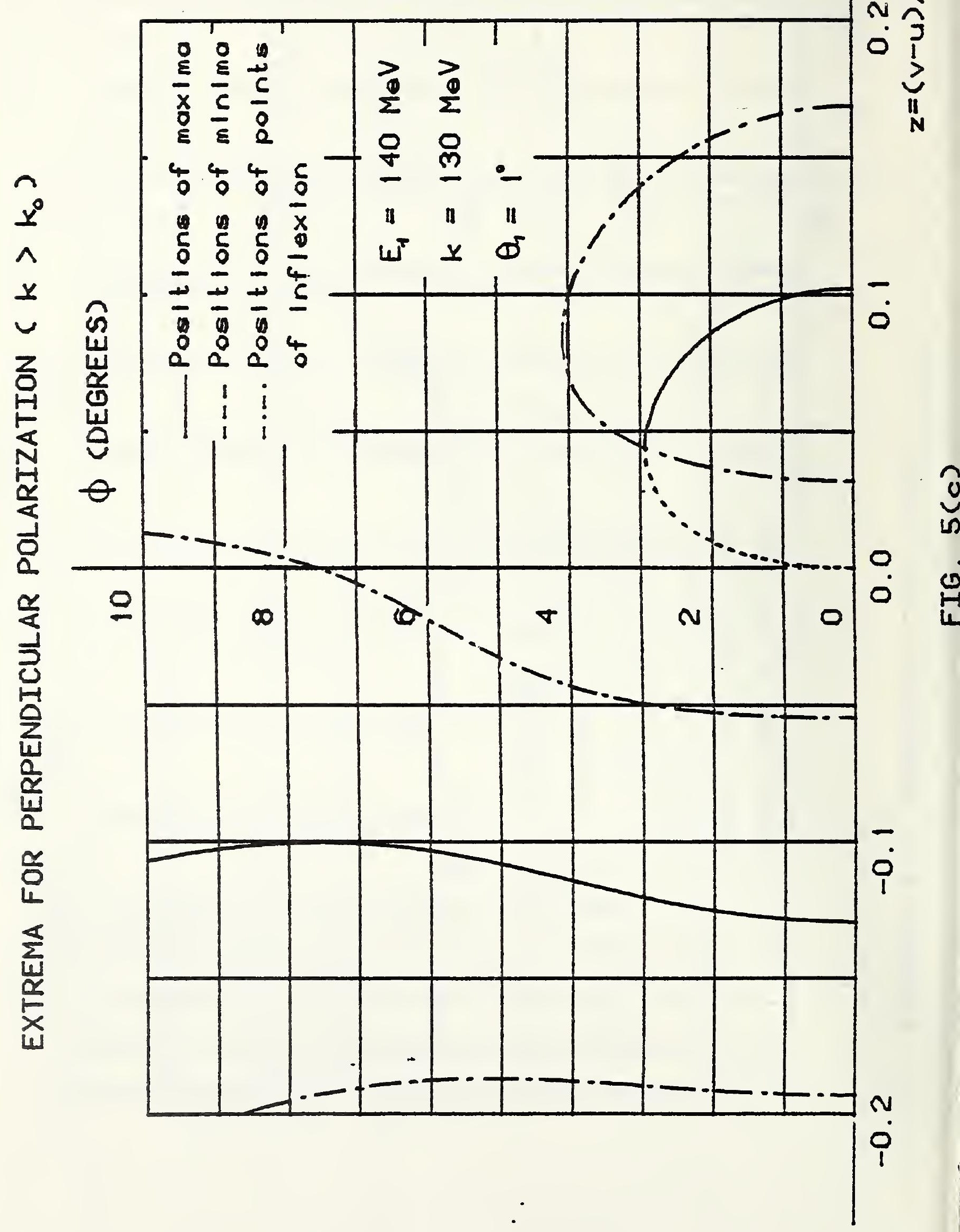




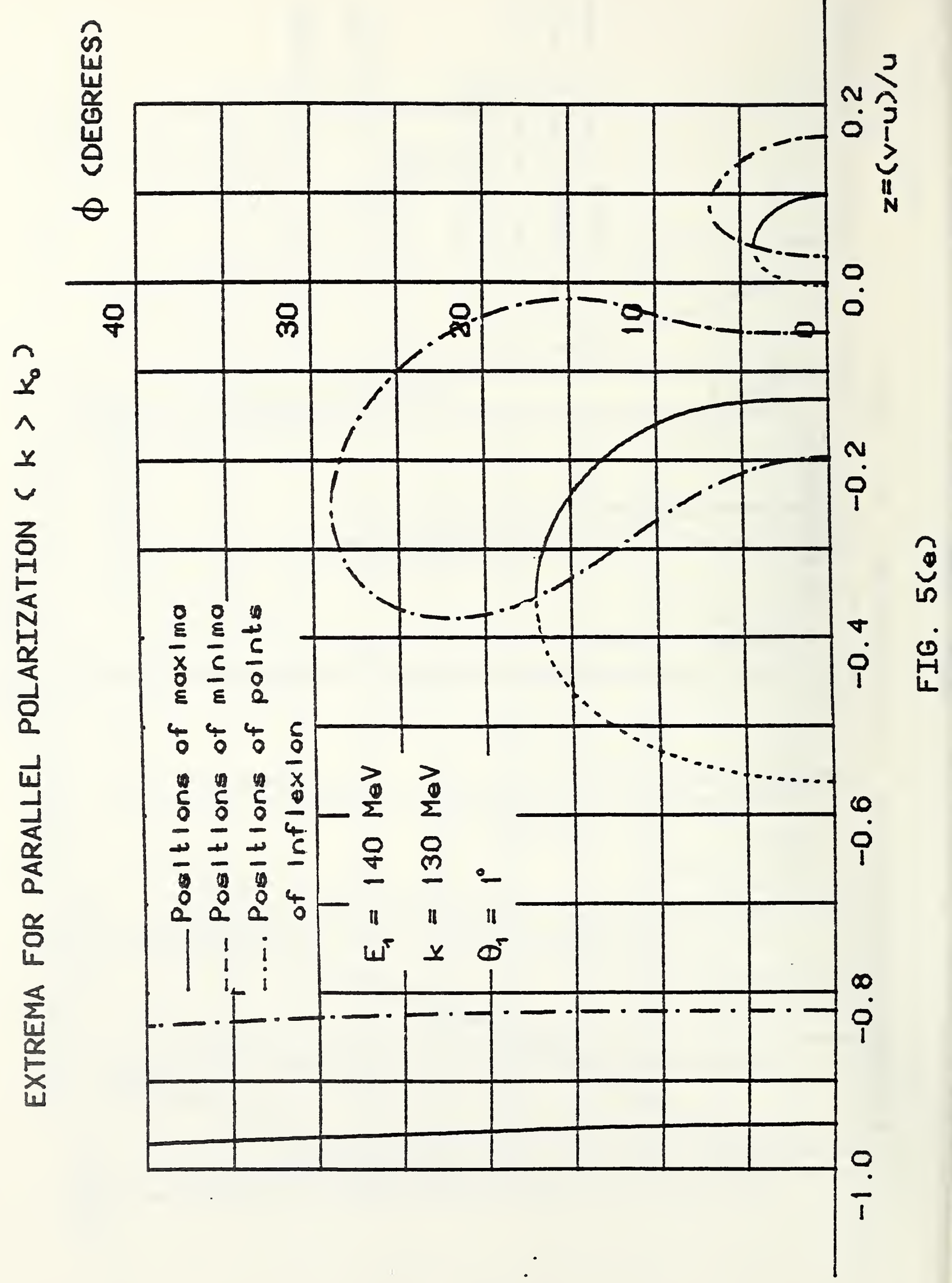




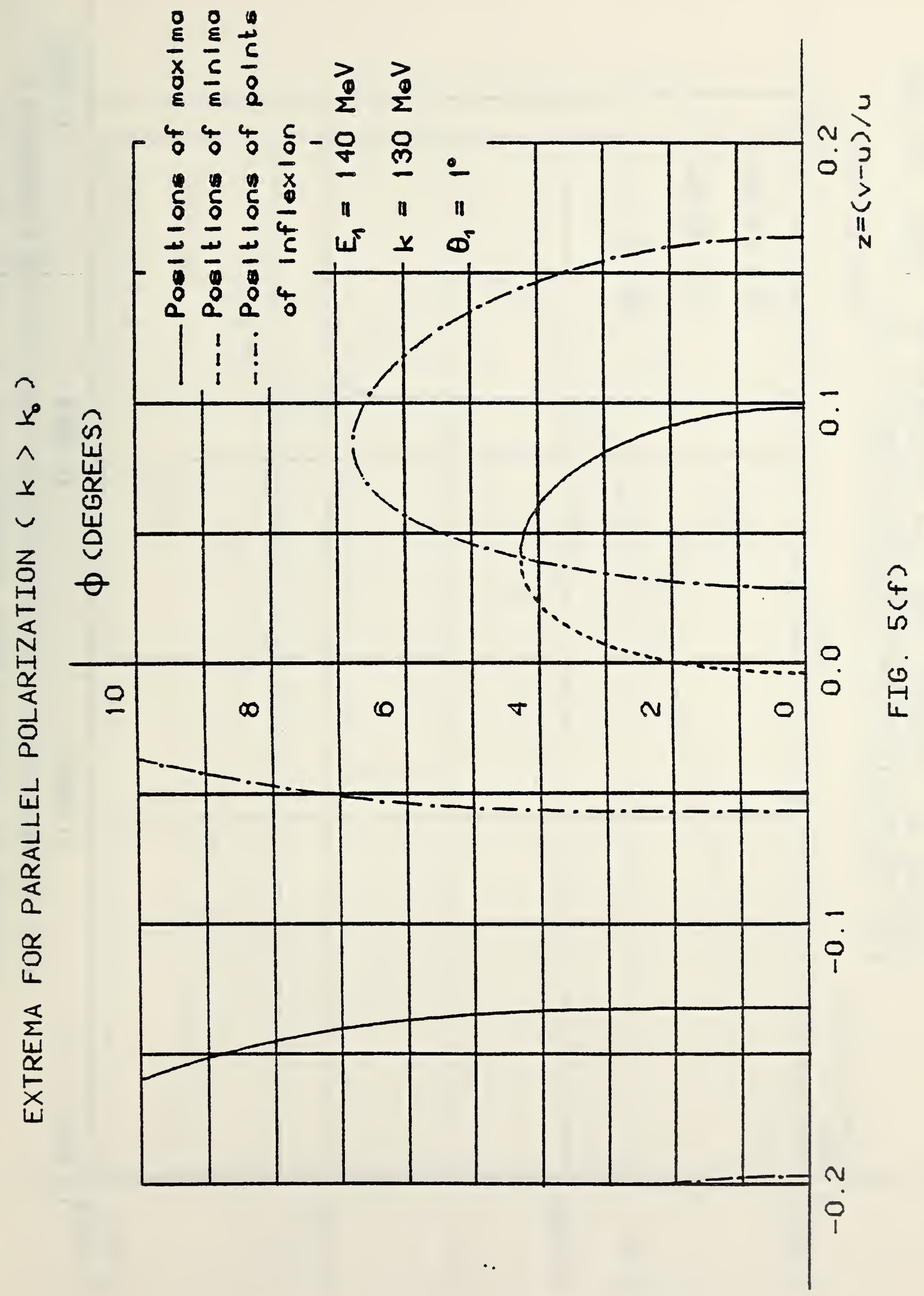




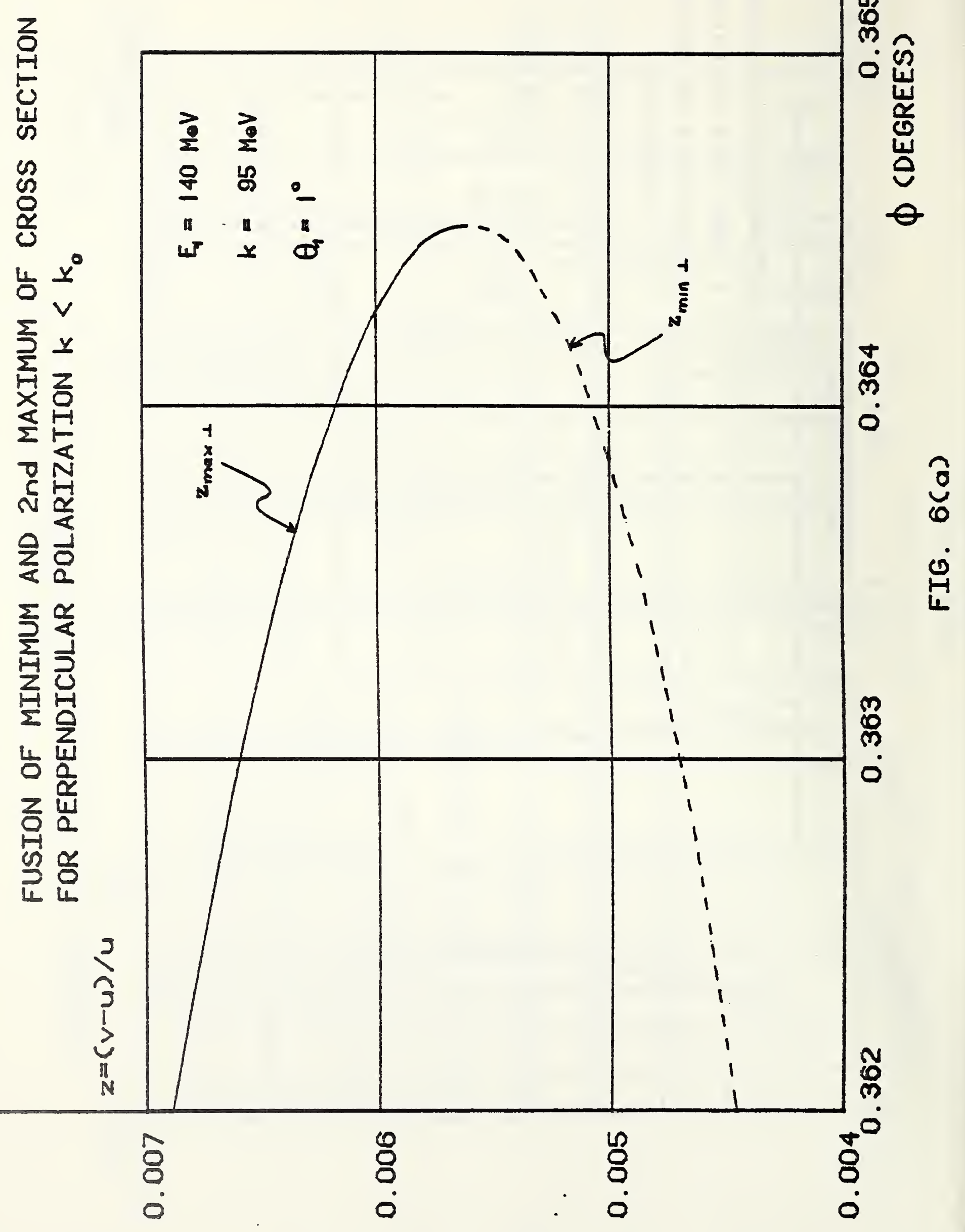




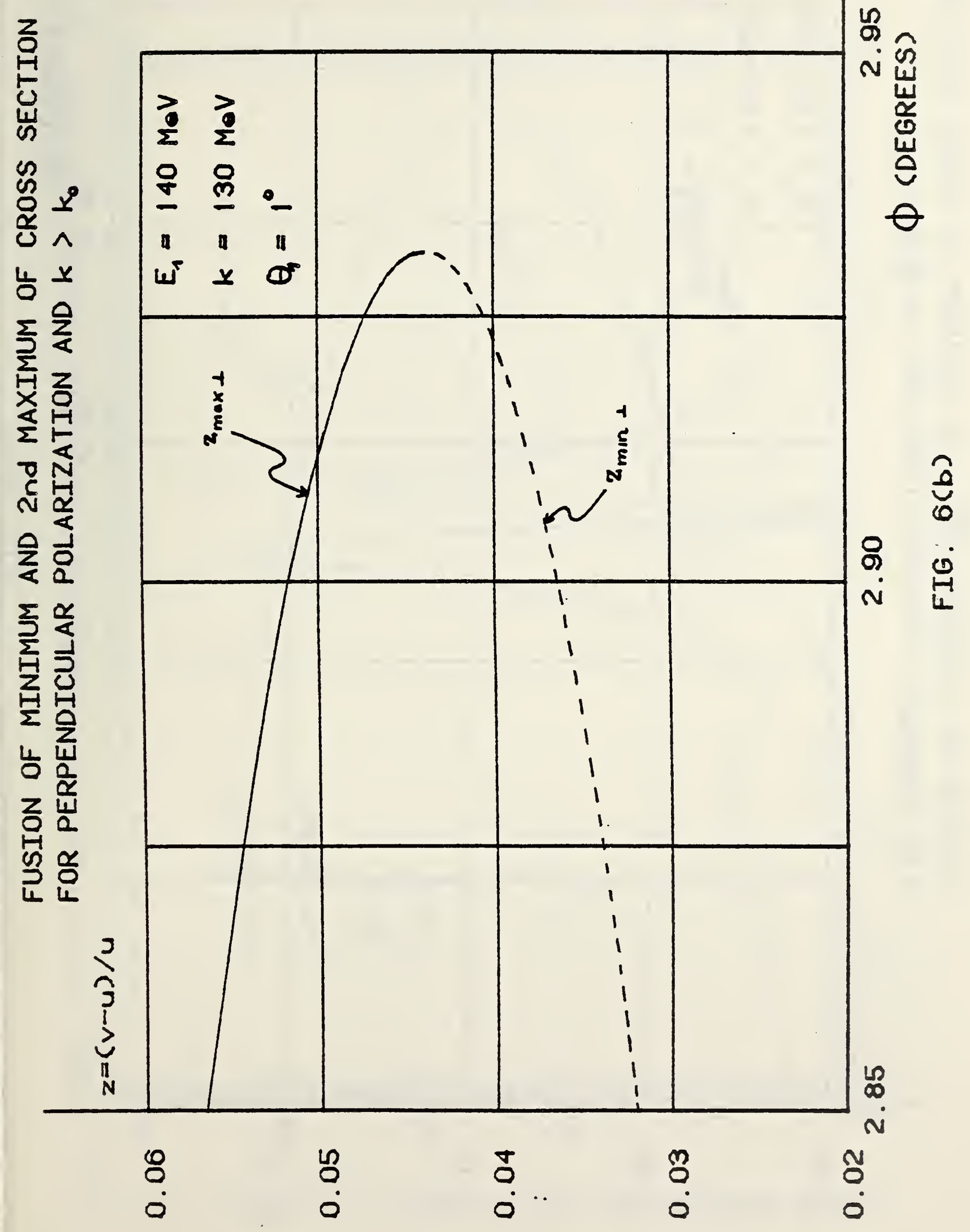




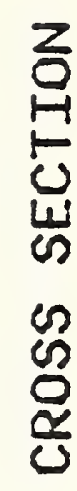

$\frac{1}{0} \uparrow$

$\sum_{\sum} \hat{y}$

范余

은

$2 \frac{1}{N}$
$\sum \frac{\alpha}{4}$
$\sum_{H} \frac{1}{0}$
0

严步

岁 $\frac{J}{\widehat{\alpha}}$

공

占产

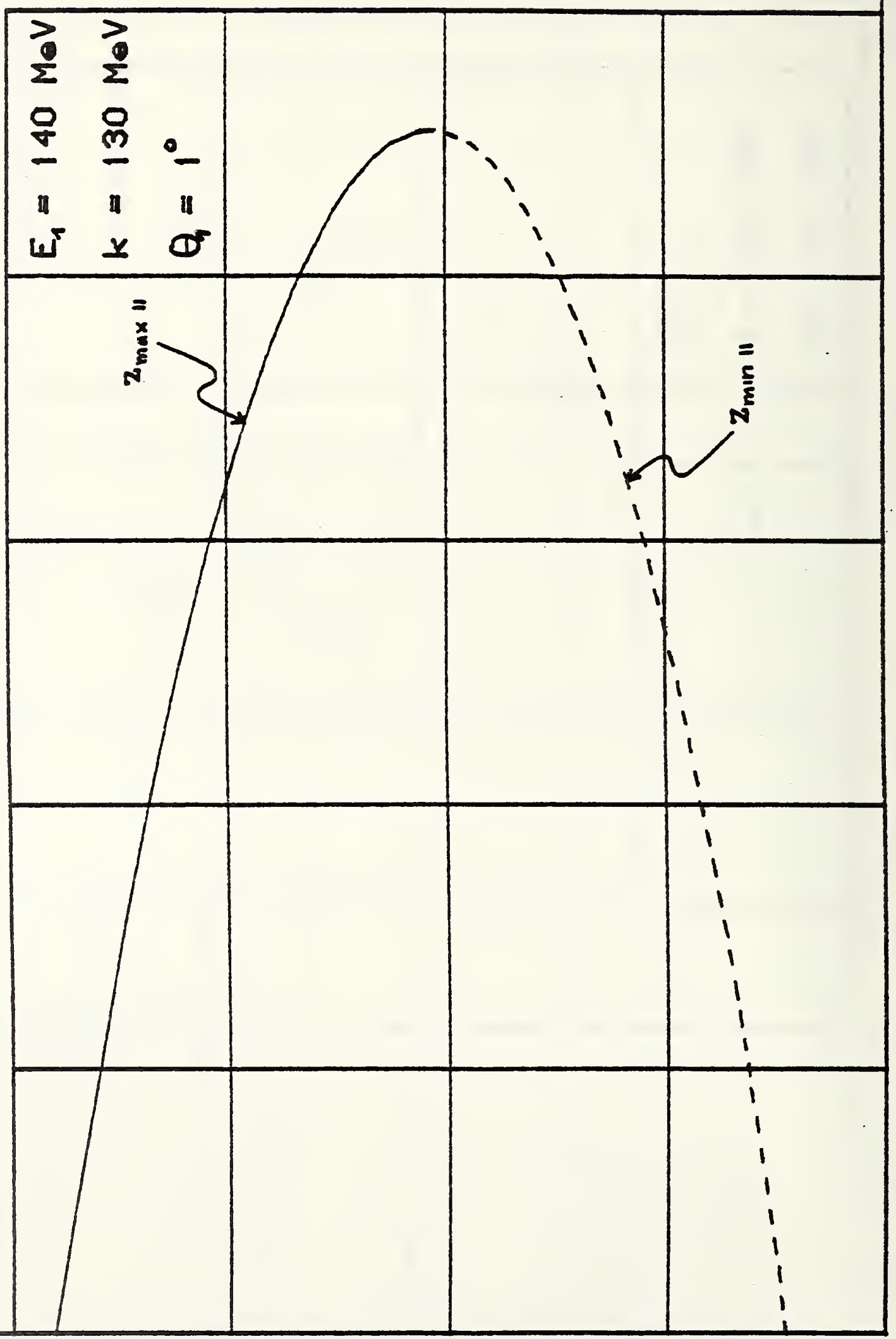

m

$\nabla$

岁

N

$\dot{\nabla}$

$\stackrel{0}{\sim}$

운

$\frac{10}{8}$

$\stackrel{0}{\circ}$

Lீ
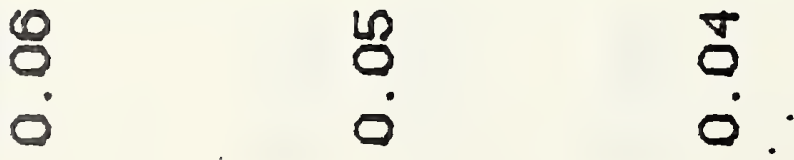

:

ปั 


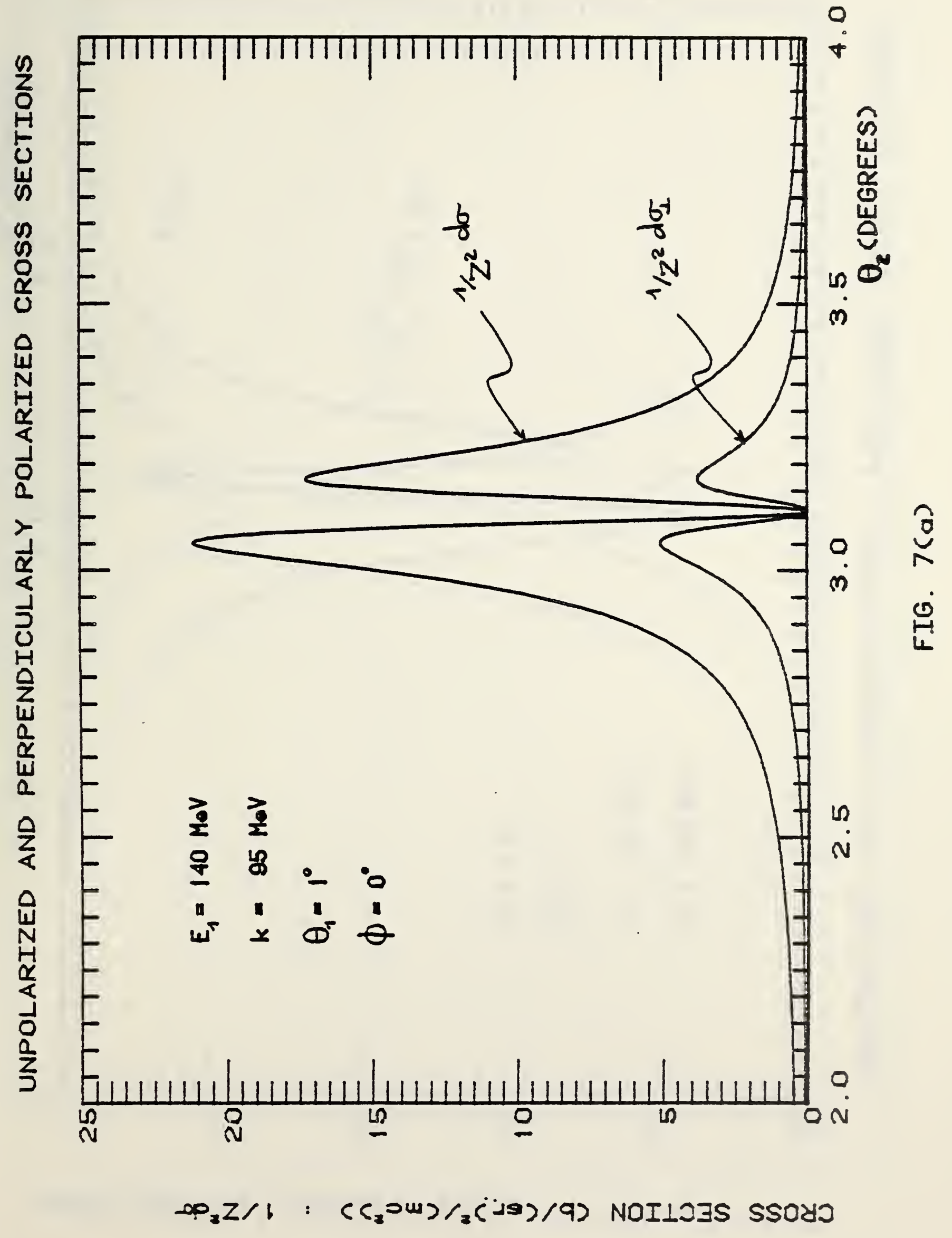




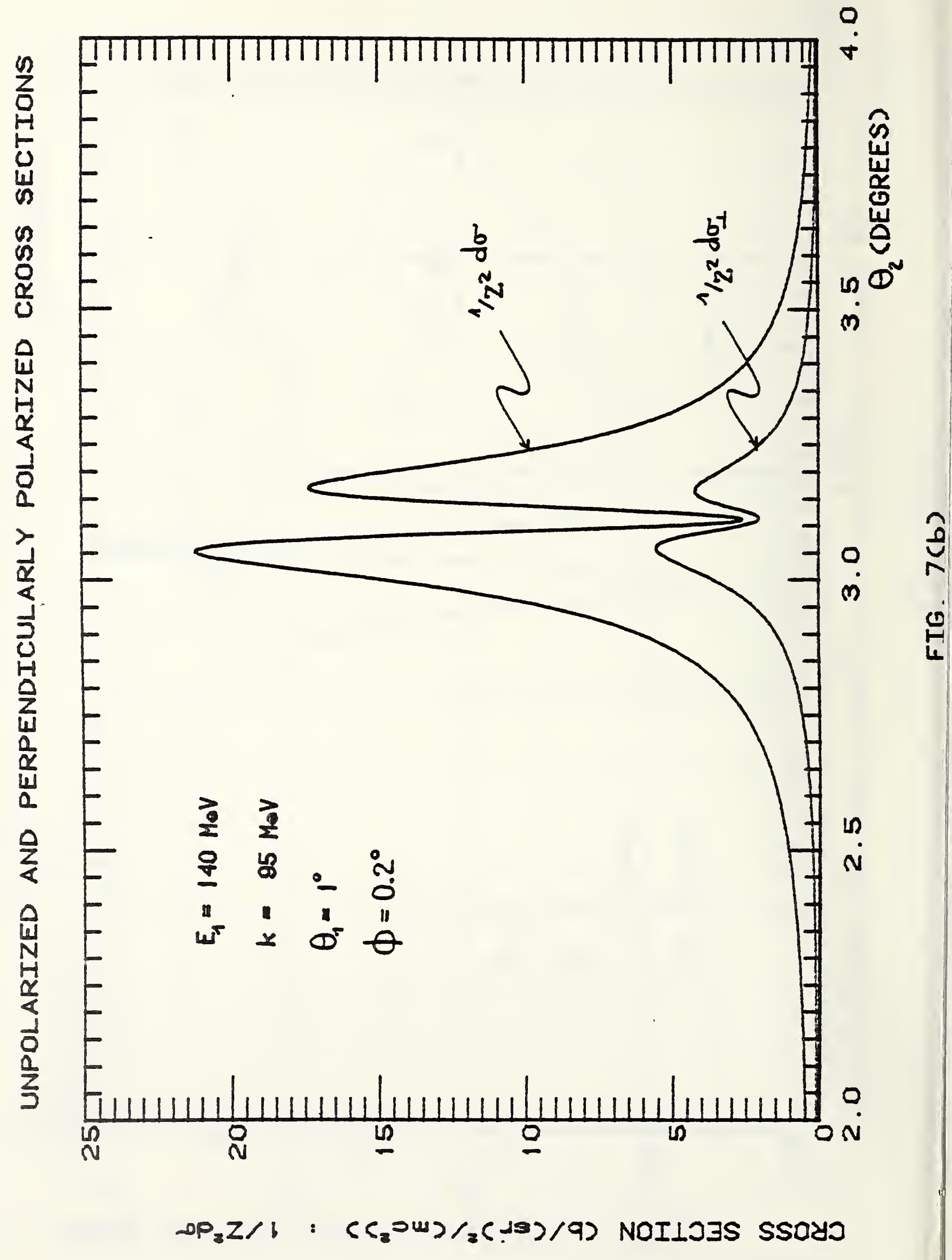




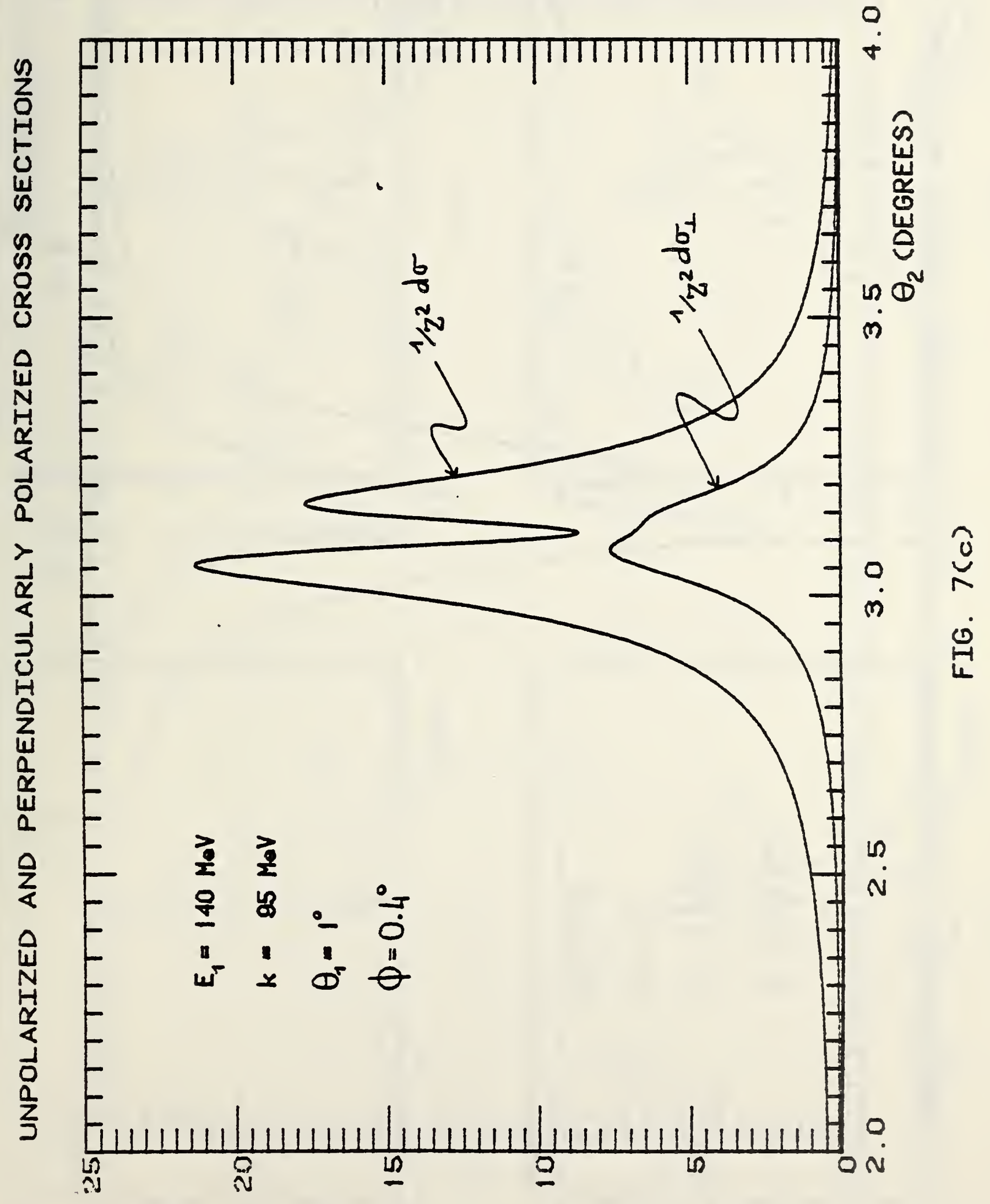

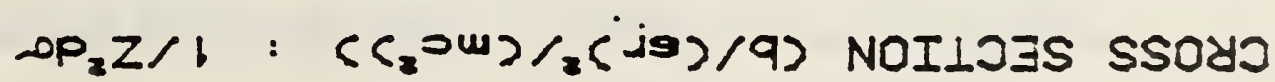




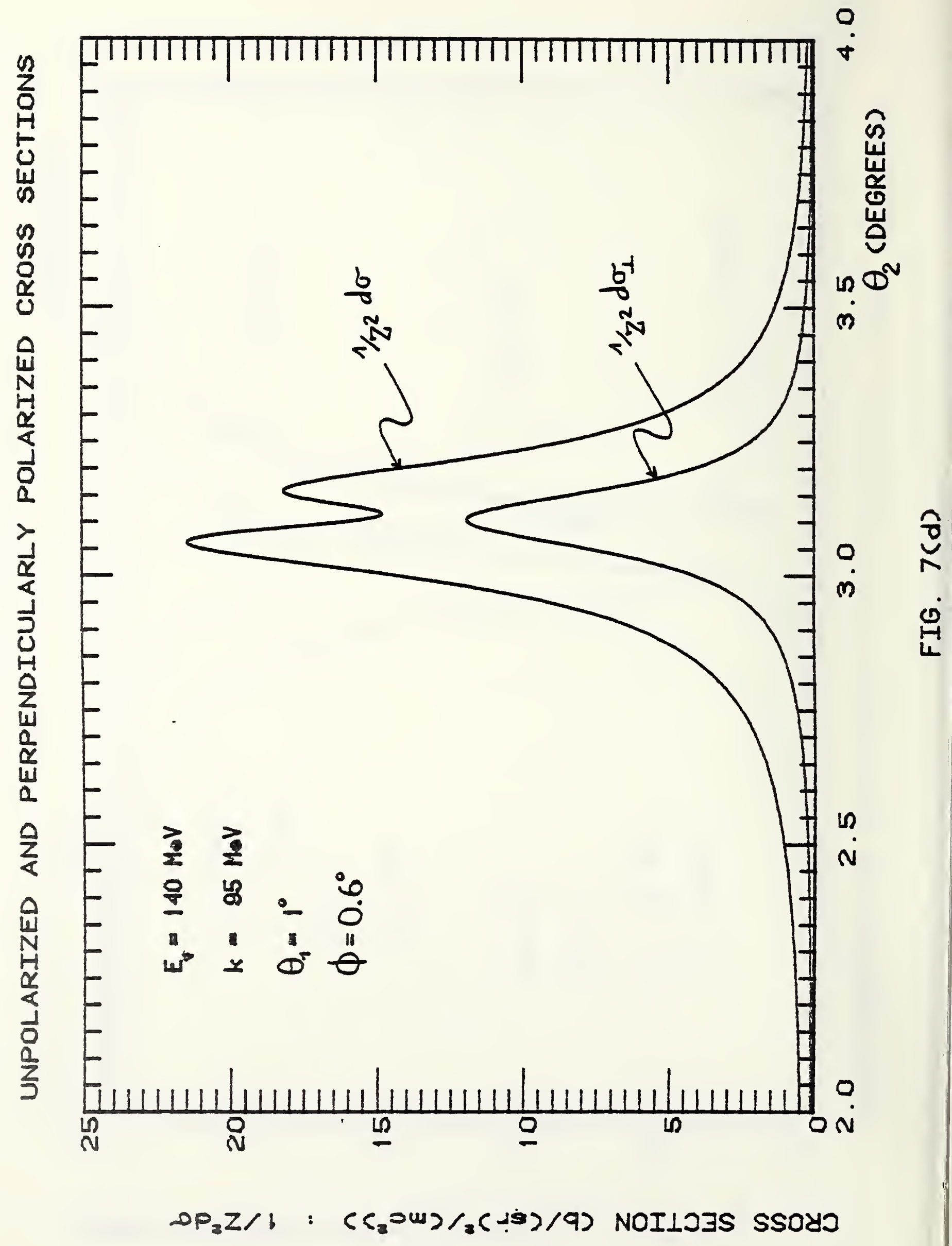



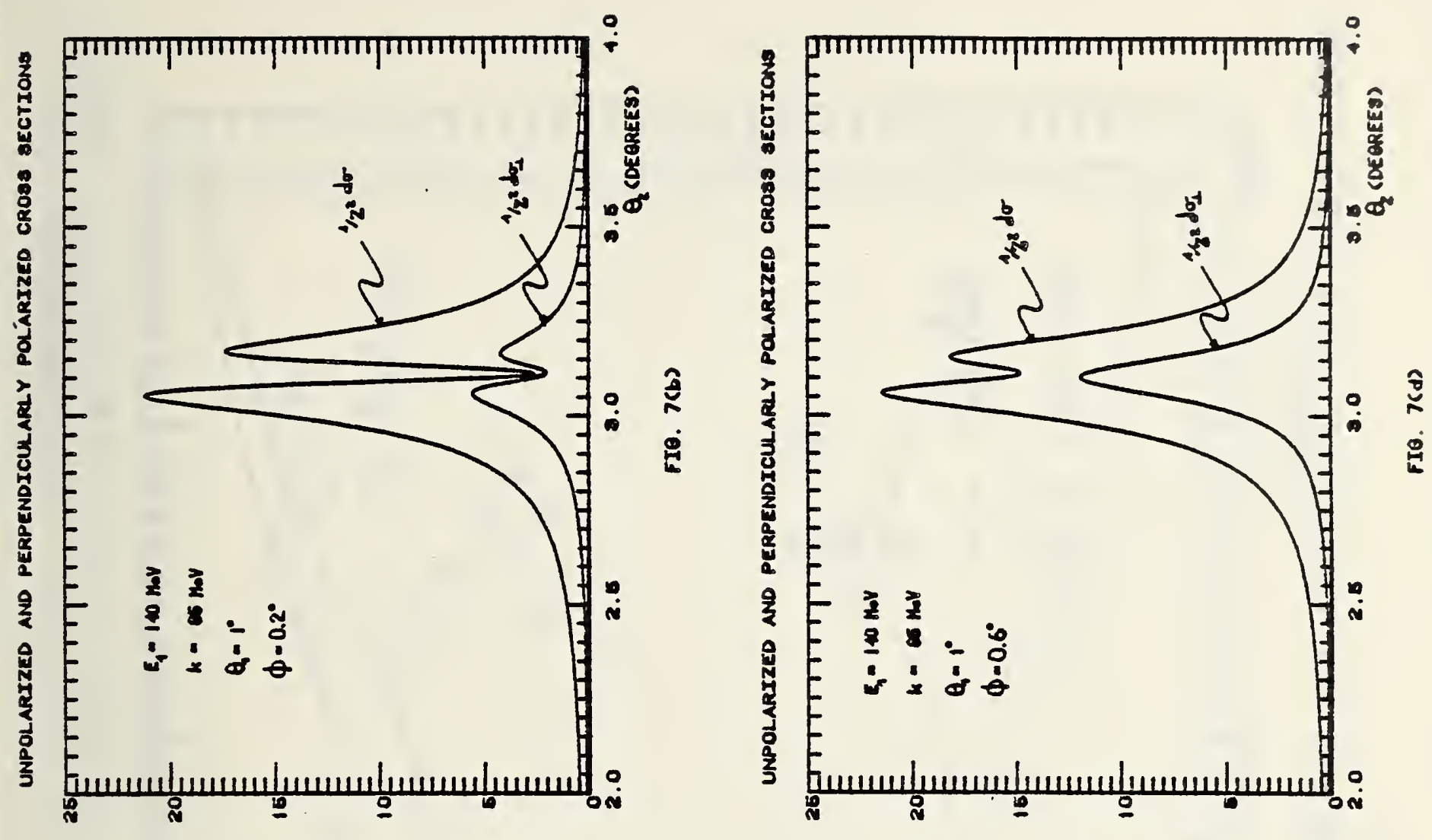

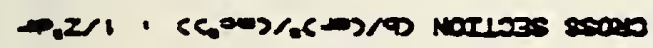

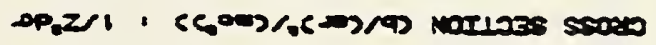
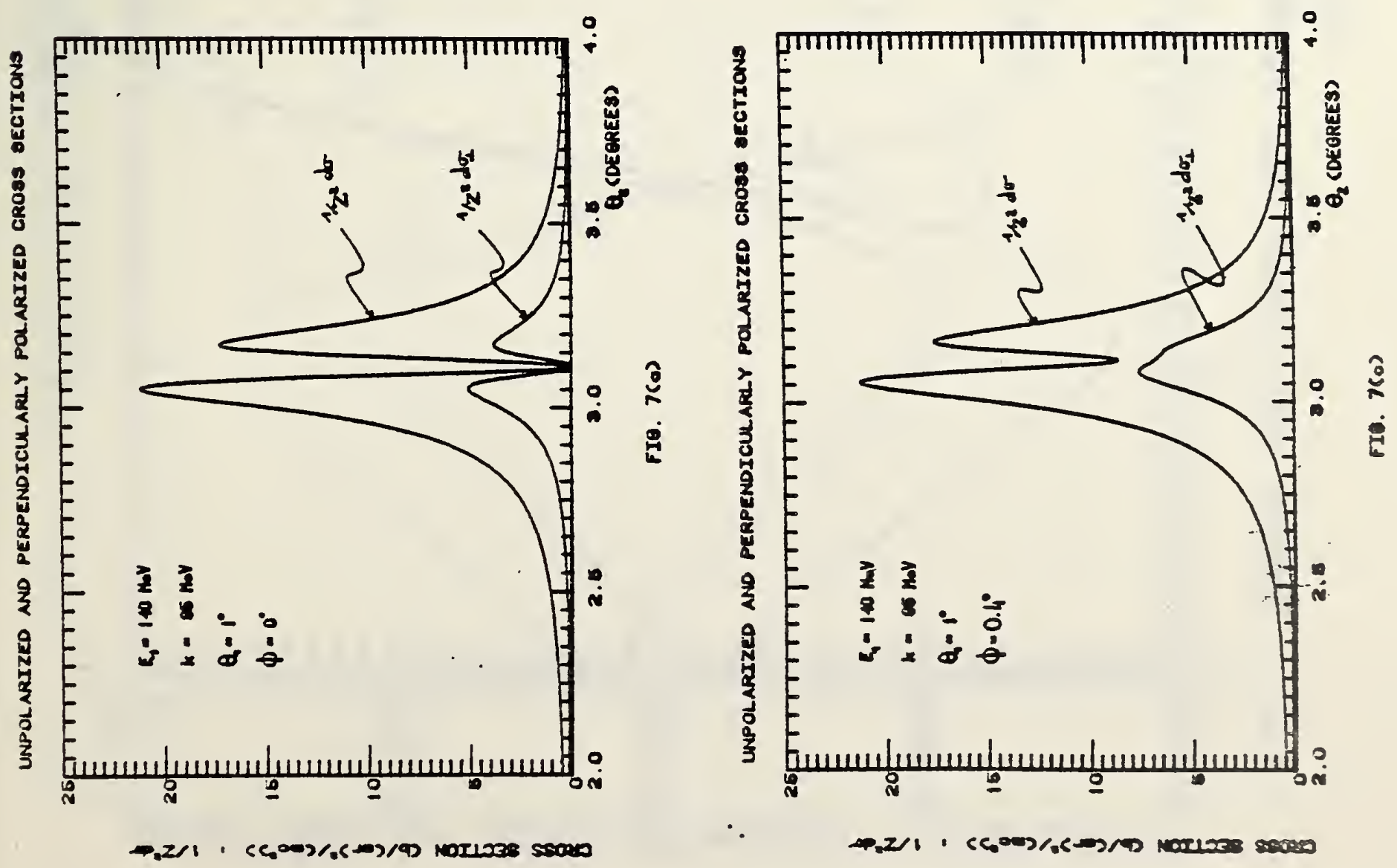


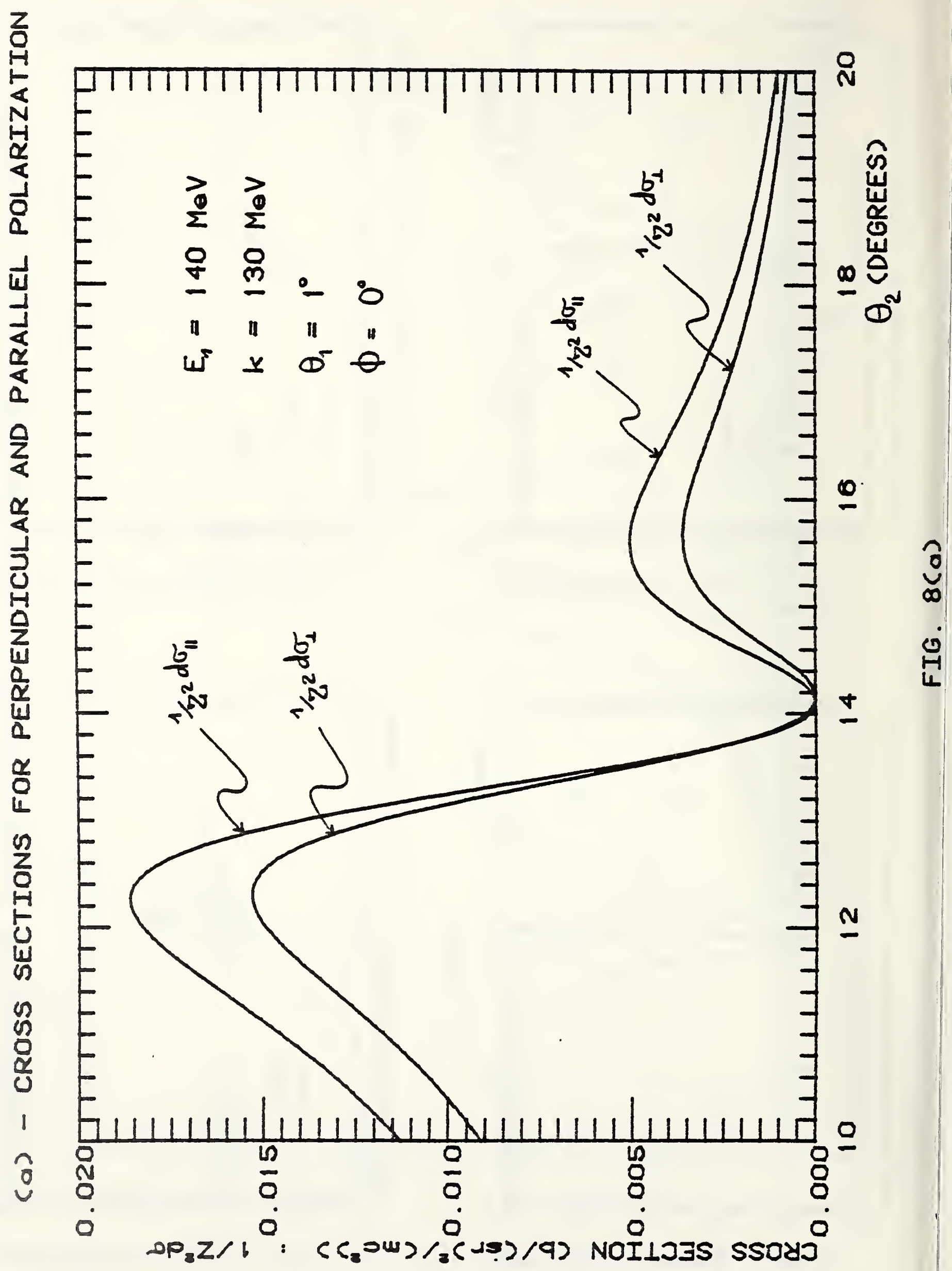




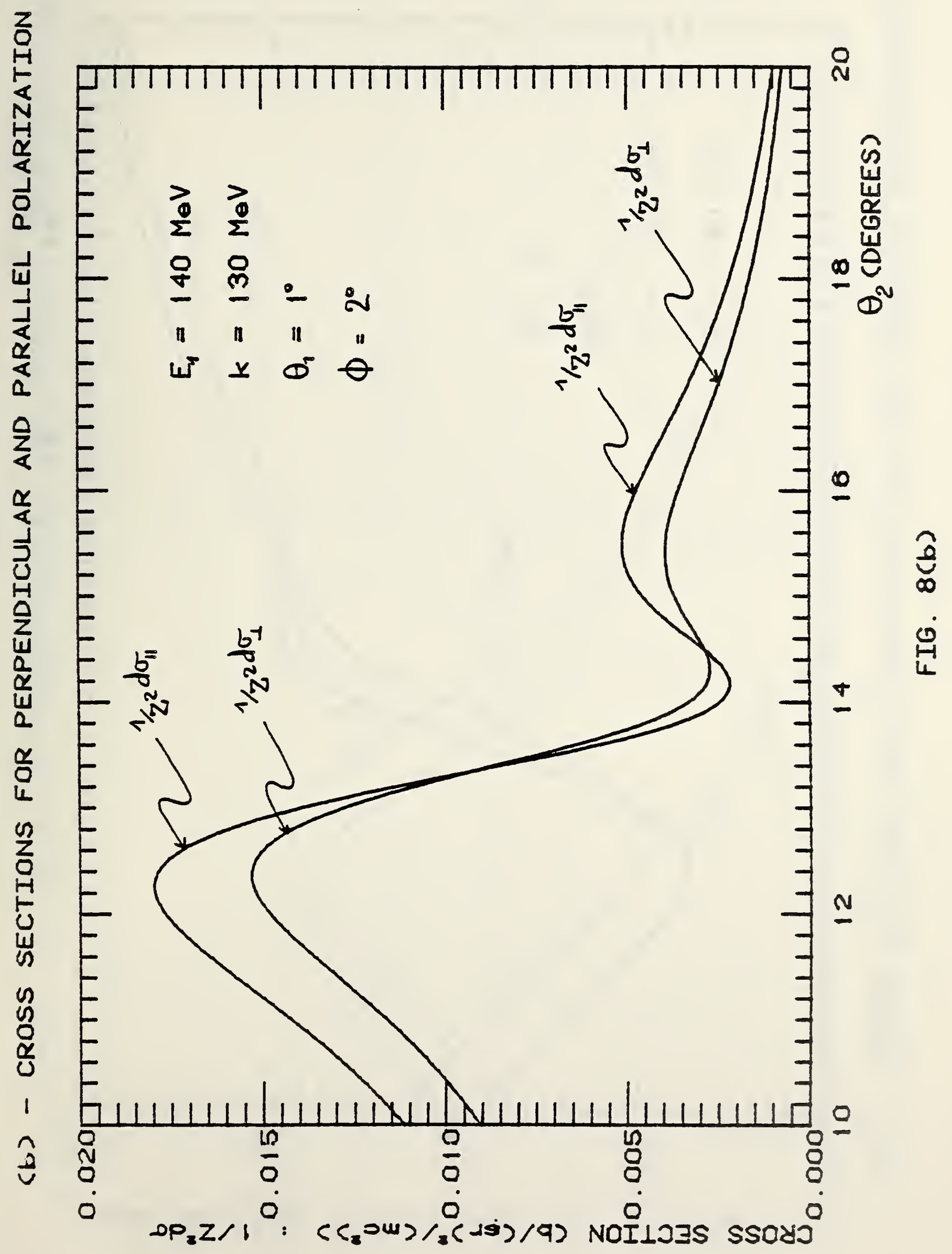




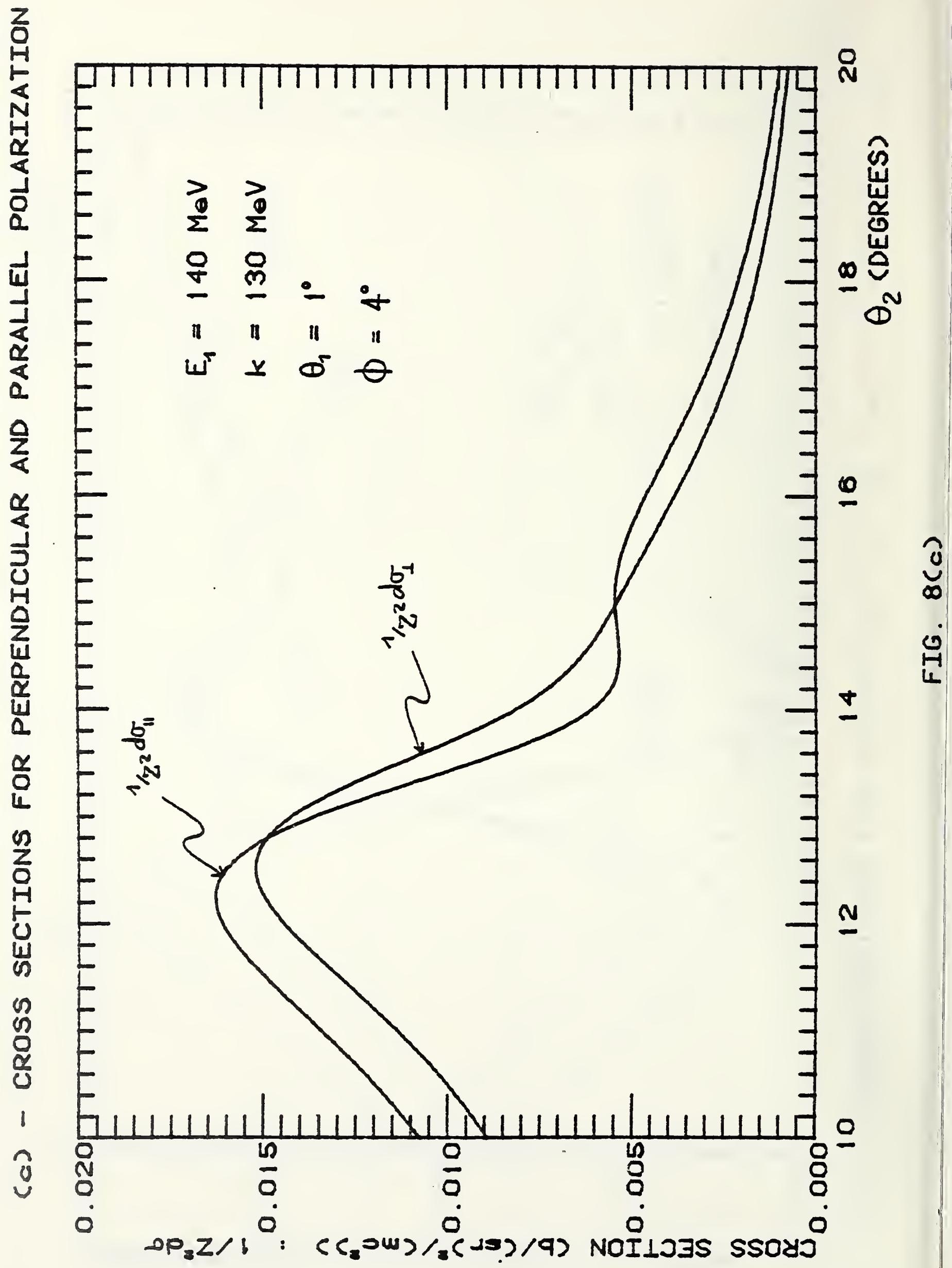




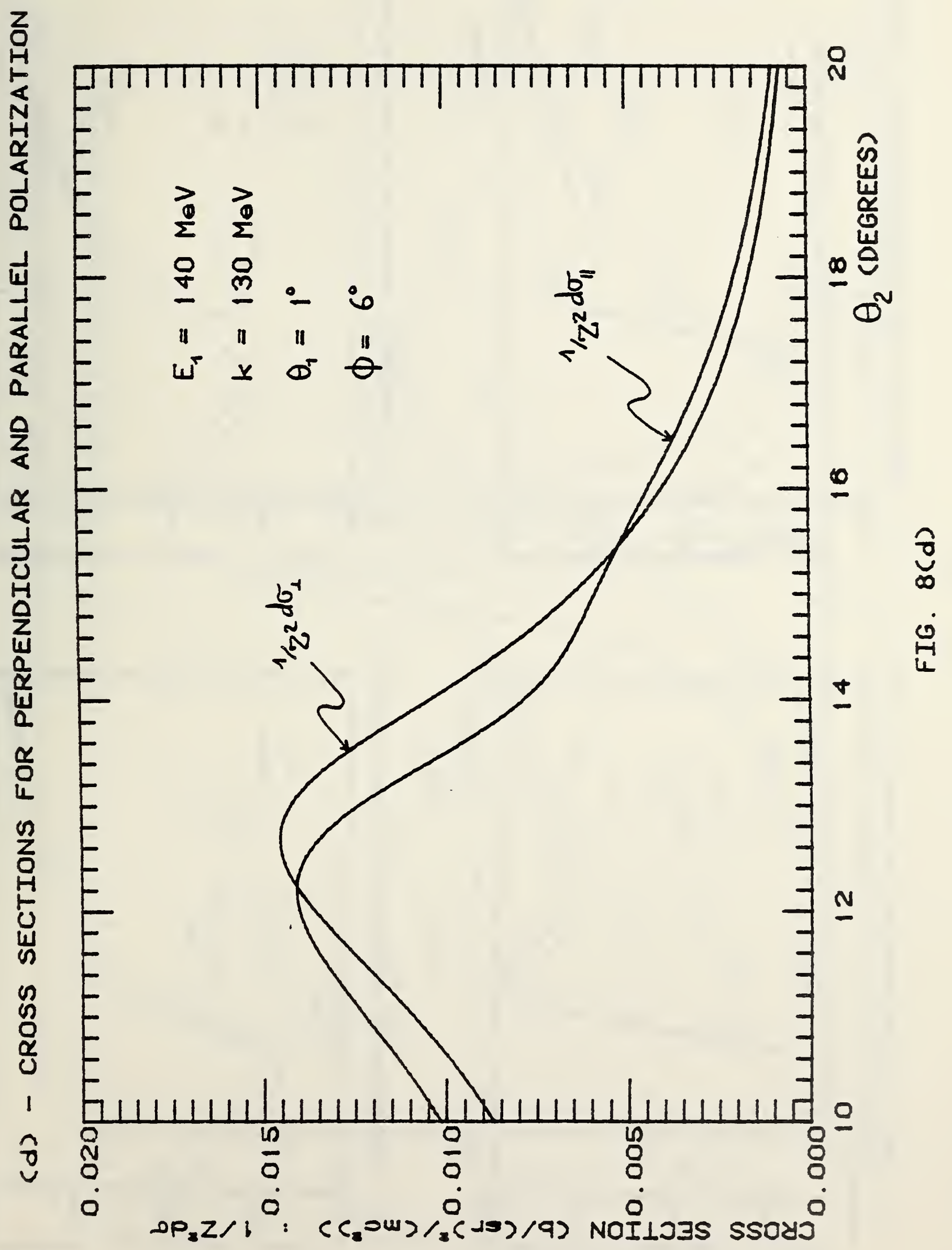



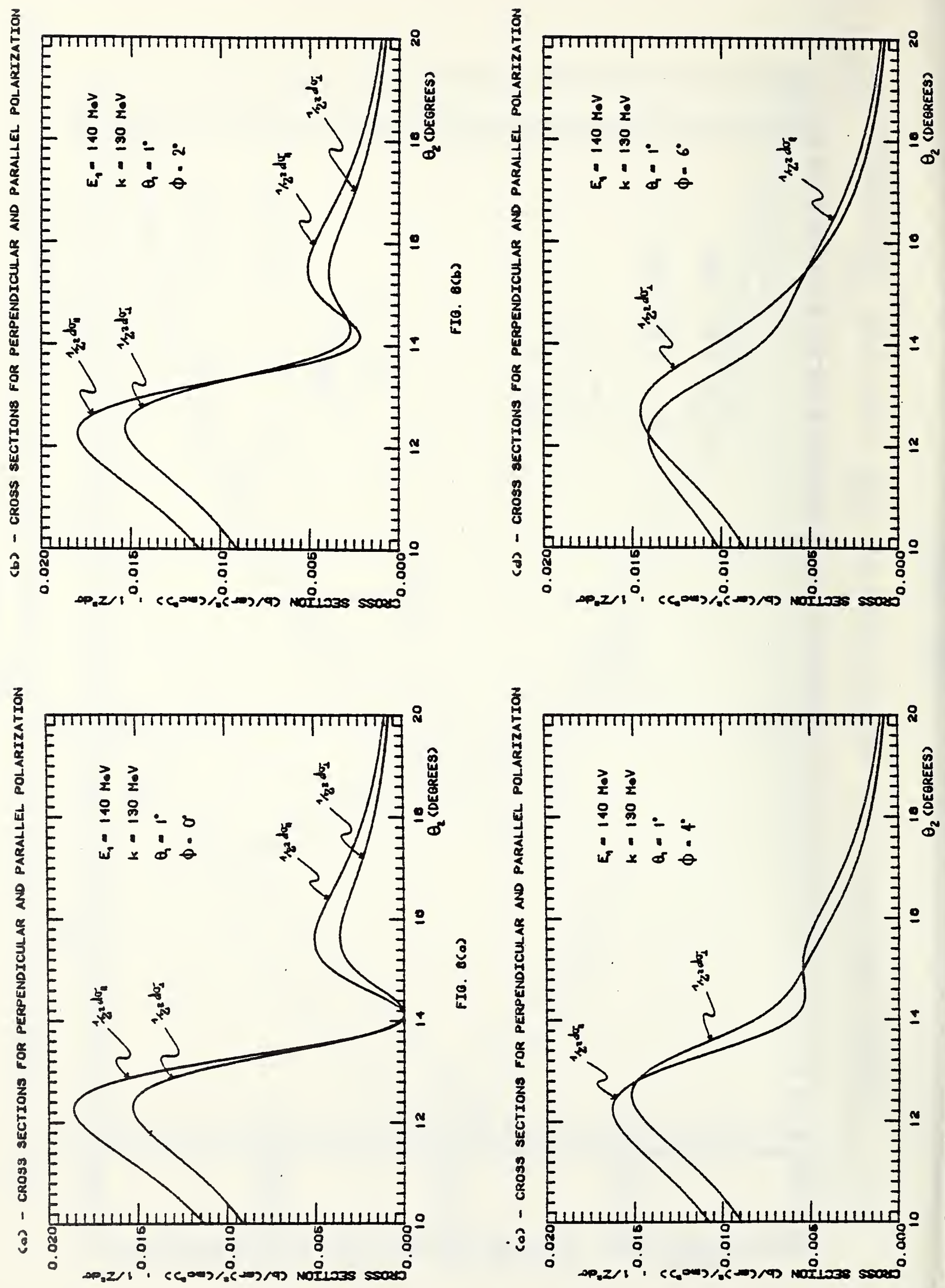


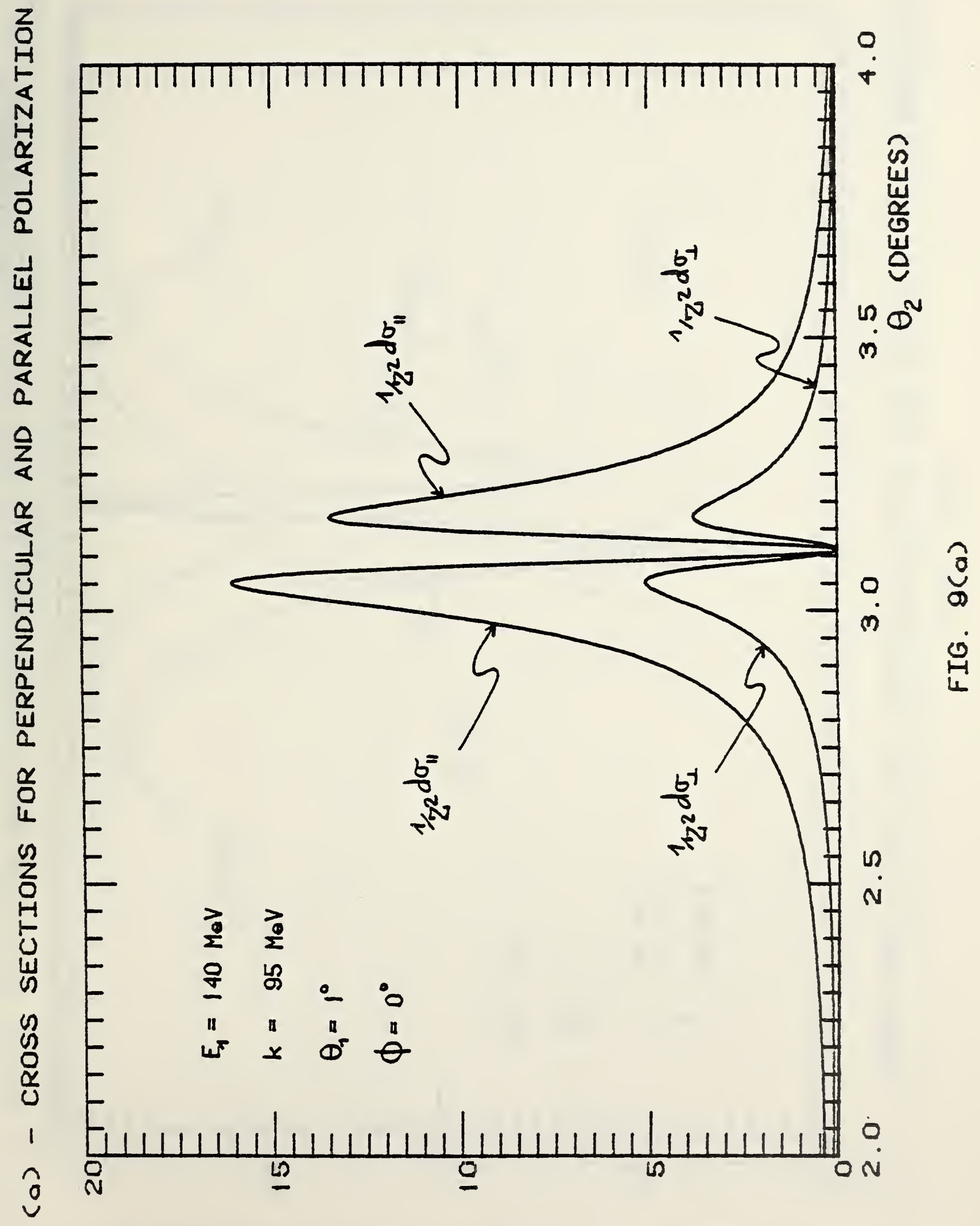

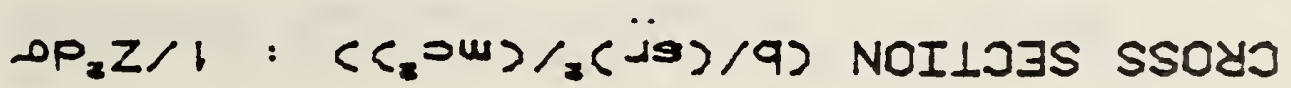




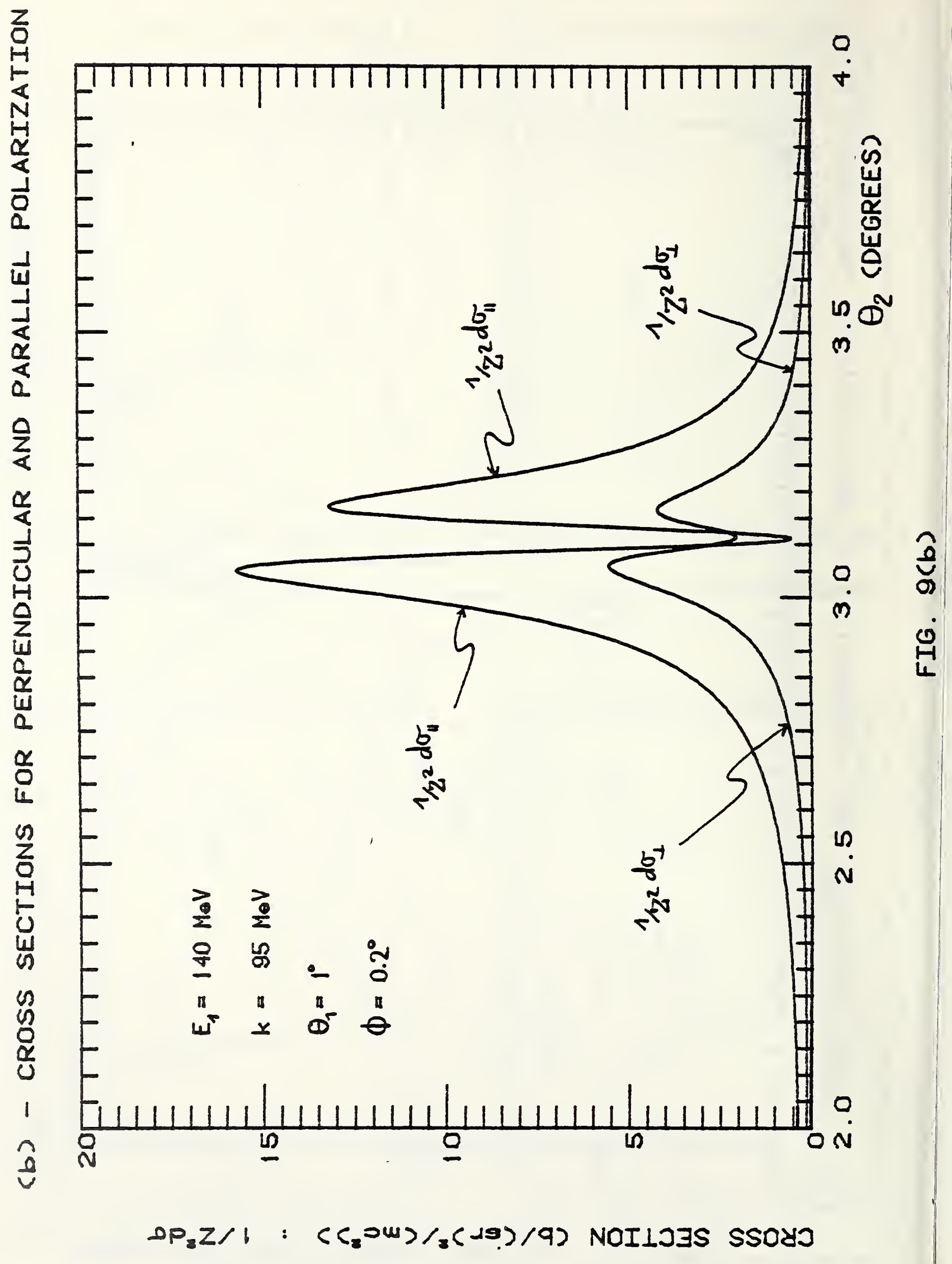




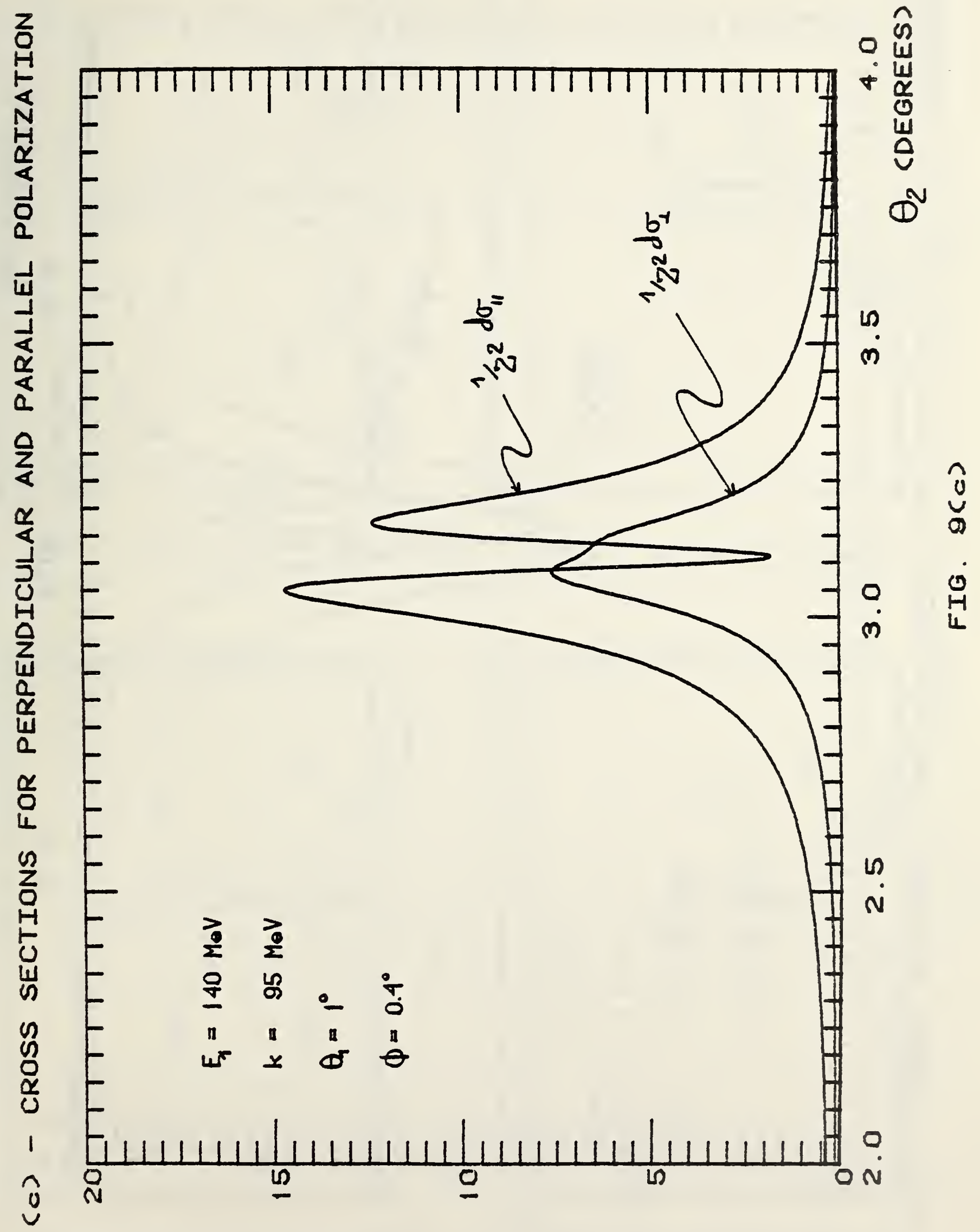

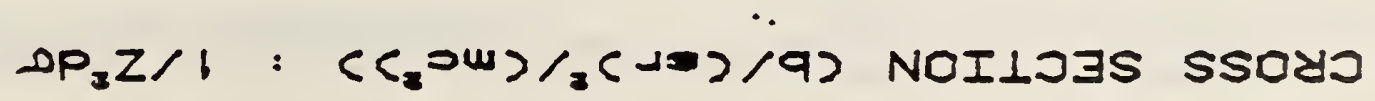




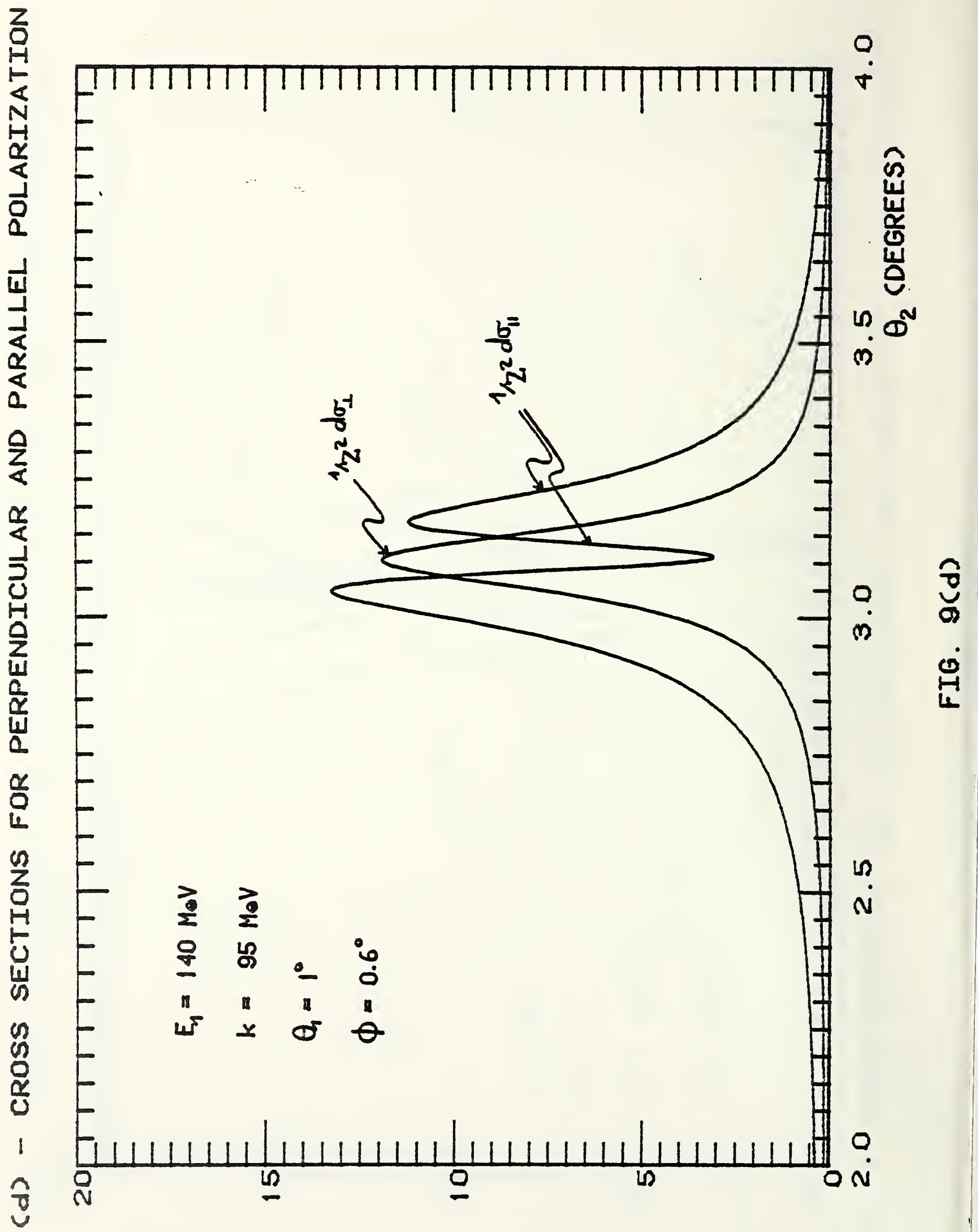

$D p_{3} Z / b:\left(C_{3}=W 2 / 3(\lambda=2 / 9)\right.$ NOILJJS SSOYJ 

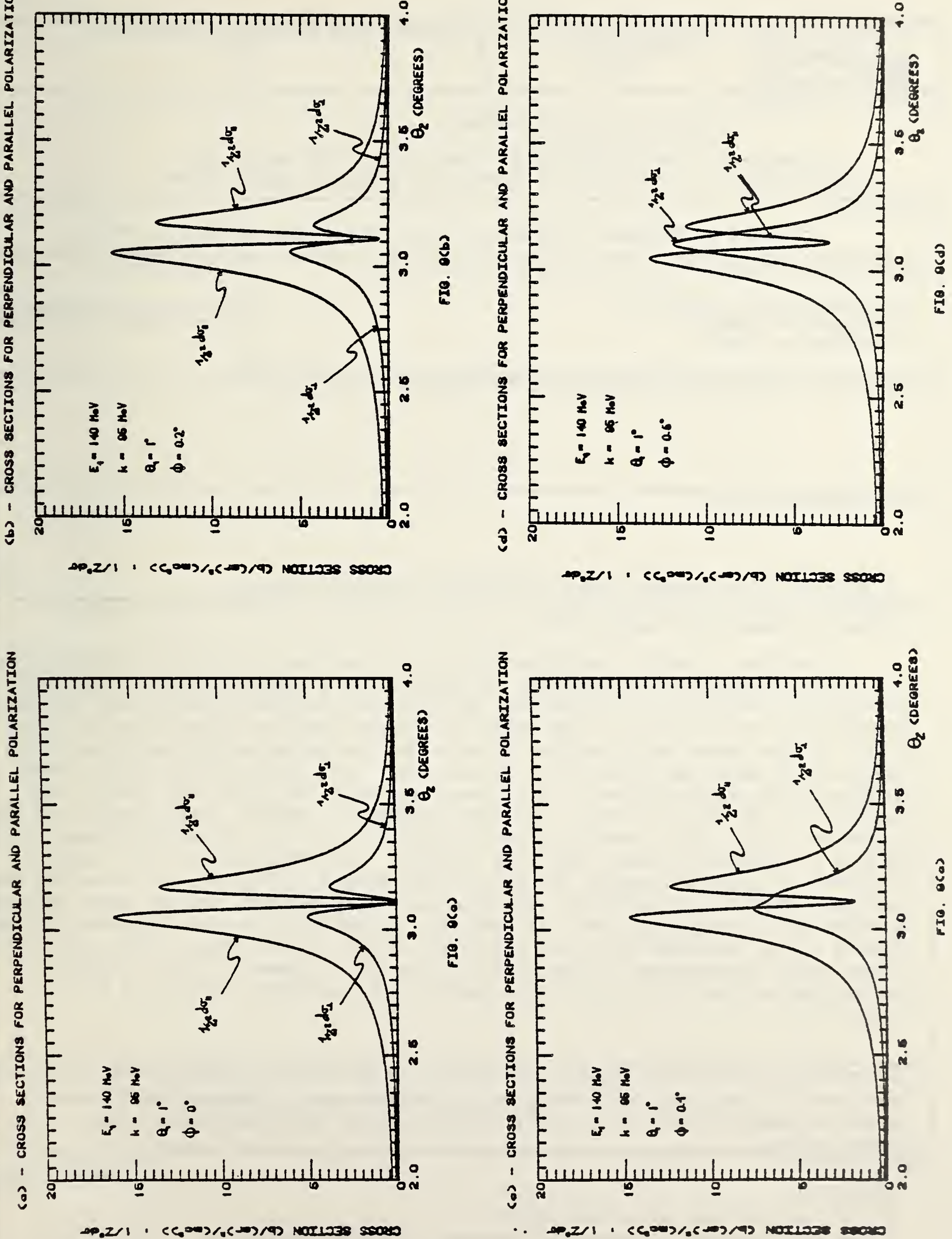

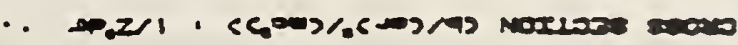


BIBLIOGRAPHIC DATA

SHEET (See instructions)

1. PUBLICATION OR REPORT NO.

$82-2454$

4. TITLE AND SUBTITLE

\section{POLARIZED TAGGED PHOTONS}

An analysis of the differential cross section for polarized

bremsstrahlung in the range of interest for a tagged photon system

5. AUTHOR(S)

Leonard C. Maximon, Eric Ganz, Thierry Aniel, and Arlette de Miniac

6. PERFORMING ORGANIZATION (If joint or other than NBS, see in structions)

MATIOHAL BUREAU OF STANDARDS

DEPARTUENT OF COMMERCE

WASHINGTON, O.C. 20234

9. SPONSORING ORGANIZATION NAME AND COMPLETE ADDRESS (Street, City, Stote, ZIP)

7. Contrace/Grant No.

8. Type of Report \& Period Covered

10. SUPPLEMENTARY NOTES

Document describes a computer program; SF-185, FiPS Software Summary, is attached.

11. ABSTRACT (A 200-word or less foctual summary of most significant information. If document includes a significant bibliography or literature survey, mention it here)

We consider in detail the differential cross section for polarized bremsstrahlung for angles and energies in the range of interest for a tagging system and derive a high energy, small angle approximation for this cross section. For photons polarized perpendicular and parallel to the production plane these are given by eqs (I.2) and (I.3). We use these approximations to determine the maxima and minima of the cross sections for these two polarization states, $d \sigma_{1}$ and $d \sigma_{\|}$, and to evaluate these cross sections at the extrema. It is shown that both do $\sigma_{1}$ and do" have a very sharp dip in the region of small momentum transfers. However, their behavior in the region of the dip, as a function of the azimuthal angle $\phi$, is quite different over most of the photon spectrum (condition (VI.33)). The cross section do 1 behaves similarly to the cross section for unpolarized photons in that as $\phi$ increases, the sharp dip vanishes, the minimum fuses with the second maximum, and the cross section then has only a single maximum. In contrast, the shart dip in the cross section do remains as $\phi$ increases, provided condition (VI.33) is satisfied. This results in rather large polarizations in the region of the dip as shown in figs. $3(a)-3(h)$. Coulomb corrections to the Born approximation are considered, and do not fill in these dips.

12. KEY WORDS (Six to twelve entries; alphabetical order; capitalize only proper names; and separate key words by semicolons) Bethe-Heitler cross section; bremsstrahlung monochromator; photonuclear research; polarized bremsstrahlung differential cross section; polarized photon beams; tagged photon method.

13. AVAILABILITY

XX Unlimited

Z For Official Distribution. Do Not Release to NTIS

Order From Superintendent of Documents, U.S. Government Printing Office, Washington, D.C. 20402.

14. NO. OF PRINTED PAGES

- Order From National Technical Information Service (NTIS). Springfield, VA. 2216I

127

15. Price

13.50 


\section{.}


